\title{
Asymptotic Spectra of Matrix-Valued Functions of Independent Random Matrices and Free Probability
}

\author{
F. Götze ${ }^{1}$ \\ Faculty of Mathematics \\ H. Kösters ${ }^{1}$ \\ University of Bielefeld \\ Faculty of Mathematics \\ University of Bielefeld \\ Germany \\ Germany \\ A. Tikhomirov ${ }^{1,2}$ \\ Department of Mathematics \\ Komi Research Center of Ural Branch of RAS, \\ Syktyvkar State University \\ Russia
}

August 13, 2014

\begin{abstract}
We investigate the universality of singular value and eigenvalue distributions of matrix valued functions of independent random matrices and apply these general results in several examples. In particular we determine the limit distribution and prove universality under general conditions for singular value and eigenvalue distributions of products of independent matrices from spherical ensembles.
\end{abstract}

\section{Introduction}

One of the main questions studied in Random Matrix Theory is the asymptotic universality, meaning the dependence on a few global characteristics of the distribution of the matrix entries, of the distribution of spectra of random matrices when their dimension goes to infinity. This holds for the spectra of Hermitian random matrices with independent entries (up to symmetry), first proved by Wigner in 1955 [48. Another well studied case is

\footnotetext{
${ }^{1}$ Partially supported by CRC 701 "Spectral Structures and Topological Methods in Mathematics", Bielefeld. ${ }^{2}$ Partially supported by RFBR, grant N 14-01-00500 and by Program of Fundamental Research Ural Division of RAS 12-P-1-1013.
} 
that of sample covariance matrices (i.e. $\mathbf{W}=\mathbf{X X}^{*}$, where $\mathbf{X}$ is a matrix with independent entries), first studied in [31] by Marchenko-Pastur. The spectrum of non Hermitian random matrices with independent identically distributed entries is universal as well. The limiting complex spectrum of this Ginibre-Girko Ensemble is the circular law (i.e. the uniform distribution on the unit circle in the complex plane). The universality here was first proved in 22] by Girko. In the last years different models of random matrices which were derived from Wigner and Ginibre-Girko matrices were studied. For instance, in [2], [3] the universality of the singular value distribution of powers of Ginibre-Girko matrices was shown. In [25] and [38] the universality of the spectrum of products of independent random matrices from the Ginibre-Girko Ensemble was proved. Moreover, more recently, the local properties of the spectrum have also been investigated in the Gaussian case; see e.g. [1] and [29].

In this paper we describe a general approach to prove the universality of singular value and eigenvalue distributions of matrix-valued functions of independent random matrices. More precisely, we consider random matrices of the form

$$
\mathbf{F}=\mathbb{F}\left(\mathbf{X}^{(1)}, \ldots, \mathbf{X}^{(m)}\right),
$$

where $\mathbf{X}^{(1)}, \ldots \mathbf{X}^{(m)}$ are independent non-Hermitian random matrices with independent entries and $\mathbb{F}$ is a matrix-valued function. Our approach is based on the Lindeberg principle of replacing matrix entries with arbitrary distributions by matrix entries with Gaussian distributions. This approach has proved to be fruitful and is used by many authors in random matrix theory; see e.g. [17, [41], 36], 25]. To prove the universality of singular value distributions, we assume a Lindeberg-type condition for the matrix entries and a certain rank condition as well as certain smoothness conditions for the matrix-valued function; see Equations (3.1), (3.2) and (3.20) - (3.25) in Section 3. To prove the universality of eigenvalue distributions, we use Girko's principle of Hermitization (see [22]), according to which there is a close connection between the eigenvalue distribution of the (square) matrix $\mathbf{F}$ and the family of the singular value distributions of all shifted matrices $\mathbf{F}-\alpha \mathbf{I}$, with $\alpha \in \mathbb{C}$. Here we need some assumptions on the large and small singular values of the shifted matrices; see Conditions $(C 0),(C 1),(C 2)$ in Section 4 .

Furthermore, we introduce a general approach to identify the limiting eigenvalue distribution of the (square) matrix F. Our main results here show how to derive the density of the limiting eigenvalue distribution of the matrix $\mathbf{F}$ from (the $S$-transform of) its limiting singular value distribution. This derivation can be divided into two major steps:

In a first step, we derive equations for the Stieltjes transforms $g(z, \alpha)$ of the (symmetrized) singular value distributions of the shifted matrices $\mathbf{F}-\alpha \mathbf{I}$ via the $S$-transform $S(z)$ of the (symmetrized) singular value distribution of the unshifted matrix $\mathbf{F}$. The key system of equations here reads

$$
\begin{aligned}
& w(z, \alpha)=z+\frac{\widetilde{R}_{\alpha}(-g(z, \alpha))}{g(z, \alpha)} \\
& g(z, \alpha)=(1+w(z, \alpha) g(z, \alpha)) S(-(1+w(z, \alpha) g(z, \alpha)))
\end{aligned}
$$


where $w(z, \alpha)$ is an unknown auxiliary function and $\widetilde{R}_{\alpha}(z)$ is a known function. To derive this system of equations, we use the asymptotic freeness of the matrices

$$
\left[\begin{array}{cc}
\mathbf{O} & \mathbf{F} \\
\mathbf{F}^{*} & \mathbf{O}
\end{array}\right] \text { and }\left[\begin{array}{cc}
\mathbf{O} & -\alpha \mathbf{I} \\
-\bar{\alpha} \mathbf{I} & \mathbf{O}
\end{array}\right]
$$

as well as the calculus for $R$-transforms and $S$-transforms. Furthermore, we show that it is possible take the limit $z \rightarrow 0$ in (1.1). Since we are working in a quite general framework, the investigation of the existence of this limit as well as its analytic properties require some work.

In a second step, we identify the density $f$ of the limiting eigenvalue distribution of the random matrix $\mathbf{F}$ using logarithmic potential theory. The main observation here is that the function $\psi(\alpha):=-w(0, \alpha) g(0, \alpha)$ is closely related to the partial derivatives of the logarithmic potential of the limiting eigenvalue distribution. Thus, under regularity assumptions, we obtain the relation

$$
f(u, v)=\frac{1}{2 \pi|\alpha|^{2}}\left(u \frac{\partial \psi}{\partial u}+v \frac{\partial \psi}{\partial v}\right)
$$

where $u$ and $v$ denote the real and imaginary part of $\alpha$, respectively.

Let us emphasize that this identification of the limiting eigenvalue distribution is quite general. In principle, we only need the $S$-transform of the limiting singular value distribution and the asymptotic freeness of the matrices in (1.2).

In Section 8 we give several examples for applications of our main universality results (Theorems 3.2 and 4.4). The guiding principle here is (i) to establish universality and (ii) to compute the limits in the Gaussian case, using tools from free probability theory. Here we focus on a special class of matrix-valued functions, namely products of matrices or powers and inverses thereof. Although our framework should, in principle, cover more general functions as well, products of independent matrices represent a convenient class of examples in which the assumptions of our main results can be checked. For instance, the conditions $(C 0),(C 1),(C 2)$ on the large and small singular values can be deduced from existing results by Tao and $\mathrm{Vu}$ [41] and Götze and Tikhomirov [24], 25] here. Moreover, once universality is proved, it suffices to identify the limiting eigenvalue and singular value distributions in the Gaussian case. But if the random matrices $\mathbf{X}^{(1)}, \ldots, \mathbf{X}^{(m)}$ have independent standard Gaussian entries, their distributions are invariant under rotations, and the $S$-transforms of the limiting singular value distributions of their products are readily obtained using tools from free probability theory, see e.g. Voiculescu [46] or Hiai and Petz [27. From here it is possible to obtain the limiting singular value distributions and, as we have seen, the limiting eigenvalue distributions.

Our examples illustrate that our main results provide a unifying framework to derive old and new results for products of independent random matrices. In particular, we determine the limiting singular value and eigenvalue distributions for products of independent random matrices from the so-called spherical ensemble (see e.g. [32]), i.e. for products of the form $\mathbf{X}^{(1)}\left(\mathbf{X}^{(2)}\right)^{-1} \cdots \mathbf{X}^{(2 m-1)}\left(\mathbf{X}^{(2 m)}\right)^{-1}$, where $\mathbf{X}^{(1)}, \ldots, \mathbf{X}^{(2 m)}$ are independent Girko-Ginibre matrices. 


\section{Table of Contents.}

1 Introduction . . . . . . . . . . . . . . . . . . . . . 1

2 General Framework . . . . . . . . . . . . . . . . . . . . . 4

3 Universality of Singular Value Distributions of Functions

of Independent Random Matrices . . . . . . . . . . . . . . . . . . . . 6

4 Universality of Eigenvalue Distributions of Functions

of Independent Random Matrices . . . . . . . . . . . . . . . . . . . . . . . . 14

5 Asymptotic Freeness of Random Matrices . . . . . . . . . . . . . . . . . . . 19

6 Stieltjes Transforms of Spectral Limits of Shifted Matrices . . . . . . . . . . . 27

7 Density of Limiting Spectral Distribution . . . . . . . . . . . . . . . . 33

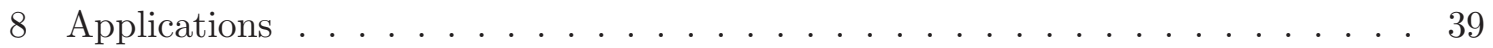

8.1 Applications of Theorem 3.2 Distribution of singular values . . . . . . . 39

8.1.1 Marchenko-Pastur Law . . . . . . . . . . . . . . . . . . . 40

8.1.2 Product of Independent Rectangular Matrices . . . . . . . . . . . . . 42

8.1.3 Powers of Random Matrices . . . . . . . . . . . . . . . . 45

8.1.4 Product of Powers of Independent Matrices . . . . . . . . . . . . . . 48

8.1.5 Polynomials of Random Matrices . . . . . . . . . . . . . . 49

8.1 .6 Spherical Ensemble . . . . . . . . . . . . . . . . . 50

8.1.7 Product of Independent Matrices from Spherical Ensemble . . . . . 58

8.2 Applications of Theorem 4.4 Distribution of eigenvalues . . . . . . . . 63

8.2.1 Circular law . . . . . . . . . . . . . . . 63

8.2.2 Product of Independent Square Matrices . . . . . . . . . . . . . . . 65

8.2.3 Product of Independent Rectangular Matrices . . . . . . . . . . . . . 66

8.2 .4 Spherical Ensemble . . . . . . . . . . . . . . . . 68

8.2.5 Product of Independent Matrices from Spherical Ensemble . . . . . 72

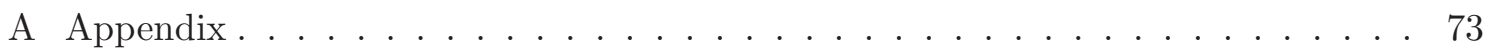

A.1 Variance of Stieltjes Transforms . . . . . . . . . . . . 73

A.2 S-Transform for Rectangular Matrices . . . . . . . . . . . . . . 74

A.3 Bounds on Singular Values . . . . . . . . . . . . . . . . 76

A.4 Technical Details for Section $7 \ldots \ldots \ldots \ldots$. . . . . . . . . . 77

\section{General Framework}

We now introduce our main assumptions and notation. Generalizations and specializations will be indicated at the beginnings of later sections.

Let $m \geq 1$ be fixed. Let $\mathcal{M}_{n \times p}$ denote the space of $n \times p$ matrices. Let $\mathbb{F}=\left(f_{j k}\right)$, $1 \leq j \leq n, 1 \leq k \leq p$ be a map from the space of $m$-tuples of $n_{0} \times n_{1}, n_{1} \times n_{2}, \ldots, n_{m-1} \times n_{m}$ matrices $\mathcal{M}_{n_{0} \times n_{1}} \times \cdots \times \mathcal{M}_{n_{m-1} \times n_{m}}$ to $\mathcal{M}_{n \times p}$. Here we assume that $n_{0}=n$ and $n_{m}=p$. 
In order to study the spectral asymptotics of sequences of such matrix tuples we shall make a so-called dimension shape assumption, meaning that $n_{q}=n_{q}(n)$, and that for any $q=1, \ldots, m$,

$$
\lim _{n \rightarrow \infty} \frac{n}{n_{q}(n)}=y_{q}>0
$$

Let $\mathbf{X}=\left(\mathbf{X}^{(1)}, \ldots, \mathbf{X}^{(m)}\right)$ be an $m$-tuple of independent random matrices of dimensions $n_{0} \times n_{1}, \ldots, n_{m-1} \times n_{m}$, respectively, with independent entries. More precisely, we assume that

$$
\mathbf{X}^{(q)}=\left(\frac{1}{\sqrt{n_{q}}} X_{j k}^{(q)}\right),
$$

where the $X_{j k}^{(q)}$ are independent complex random variables such that for all $q=1, \ldots, m$ and $j=1, \ldots, n_{q-1} ; k=1, \ldots, n_{q}$, we have $\mathbf{E} X_{j k}^{(q)}=0$ and $\mathbf{E}\left|X_{j k}^{(q)}\right|^{2}=1$.

Furthermore, let $\mathbf{Y}=\left(\mathbf{Y}^{(1)}, \ldots, \mathbf{Y}^{(m)}\right)$ be an $m$-tuple of independent random matrices of dimensions $n_{0} \times n_{1}, \ldots, n_{m-1} \times n_{m}$, respectively, with independent Gaussian entries. More precisely, we assume that

$$
\mathbf{Y}^{(q)}=\left(\frac{1}{\sqrt{n_{q}}} Y_{j k}^{(q)}\right)
$$

where the $Y_{j k}^{(q)}$ are independent complex random variables such that for all $q=1, \ldots, m$ and $j=1, \ldots, n_{j-q} ; k=1, \ldots, n_{q},\left(\operatorname{Re} Y_{j k}^{(q)}, \operatorname{Im} Y_{j k}^{(q)}\right)$ has a bivariate Gaussian distribution with the same first and second moments as $\left(\operatorname{Re} X_{j k}^{(q)}, \operatorname{Im} X_{j k}^{(q)}\right)$. By this we mean that

$$
\begin{gathered}
\mathbf{E} \operatorname{Re} Y_{j k}^{(q)}=\mathbf{E} \operatorname{Re} X_{j k}^{(q)}, \mathbf{E} \operatorname{Im} Y_{j k}^{(q)}=\mathbf{E} \operatorname{Im} X_{j k}^{(q)}, \\
\mathbf{E}\left|\operatorname{Re} Y_{j k}^{(q)}\right|^{2}=\mathbf{E}\left|\operatorname{Re} X_{j k}^{(q)}\right|^{2}, \mathbf{E}\left|\operatorname{Im} Y_{j k}^{(q)}\right|^{2}=\mathbf{E}\left|\operatorname{Im} X_{j k}^{(q)}\right|^{2}, \\
\mathbf{E}\left(\operatorname{Re} Y_{j k}^{(q)} \operatorname{Im} Y_{j k}^{(q)}\right)=\mathbf{E}\left(\operatorname{Re} X_{j k}^{(q)} \operatorname{Im} X_{j k}^{(q)}\right) .
\end{gathered}
$$

In particular, $\mathbf{E} Y_{j k}^{(q)}=0$ and $\mathbf{E}\left|Y_{j k}^{(q)}\right|^{2}=1$.

In Section 8 , when we determine the limiting singular value and eigenvalue distributions in the Gaussian case, we will impose the stronger assumption that the $Y_{j k}^{(q)}$ are standard real or complex Gaussian random variables. By Eq. (2.2), this entails some restrictions on the second moments of the $X_{j k}^{(q)}$.

We shall also assume that the random matrices $\mathbf{X}^{(1)}, \ldots, \mathbf{X}^{(m)}$ and $\mathbf{Y}^{(1)}, \ldots, \mathbf{Y}^{(m)}$ are defined on the same probability space and that $\mathbf{Y}^{(1)}, \ldots, \mathbf{Y}^{(m)}$ are independent of $\mathbf{X}^{(1)}, \ldots, \mathbf{X}^{(m)}$. Finally, for any $m$-tuple $\mathbf{Z}=\left(\mathbf{Z}^{(1)}, \ldots, \mathbf{Z}^{(m)}\right)$ in $\mathcal{M}_{n_{0} \times n_{1}} \times \cdots \times \mathcal{M}_{n_{m-1} \times n_{m}}$, we set

$$
\mathbf{F}_{\mathbf{Z}}:=\mathbb{F}\left(\mathbf{Z}^{(1)}, \ldots, \mathbf{Z}^{(m)}\right) .
$$

Note that since we are interested in asymptotic singular value and eigenvalue distributions, we are actually dealing with sequences of matrix tuples of increasing dimension. However, the dependence on $n$ is usually suppressed in our notation. 
Throughout this paper, we use the following notation. For a matrix $\mathbf{A}=\left(a_{j k}\right) \in \mathcal{M}_{n \times p}$, we write $\|\mathbf{A}\|$ for the operator norm of $\mathbf{A}$ and $\|\mathbf{A}\|_{2}:=\left(\sum_{j=1}^{n} \sum_{k=1}^{p}\left|a_{j k}\right|^{2}\right)^{1 / 2}$ for the Frobenius norm of $\mathbf{A}$. The singular values of $\mathbf{A}$ are the square-roots of the eigenvalues of the $n \times n$ matrix $\mathbf{A A}^{*}$. Finally, unless otherwise indicated, $C$ and $c$ denote sufficiently large and small positive constants, respectively, which may change from step to step.

\section{Universality of Singular Value Distributions of Functions of Independent Random Matrices}

We start with the singular value distribution of functions of independent random matrices. Let $\mathbf{X}=\left(\mathbf{X}^{(1)}, \ldots, \mathbf{X}^{(m)}\right)$ be an $m$-tuple of independent random matrices with independent entries as in Section 1, and let $\mathbf{F}_{\mathbf{X}}=\mathbb{F}\left(\mathbf{X}^{(1)}, \ldots, \mathbf{X}^{(m)}\right)$ be a matrix-valued function of $\mathbf{X}$. We are interested in the empirical distribution of the singular values of $\mathbf{F}_{\mathbf{X}}$, i.e. of the square-roots of the eigenvalues of $\mathbf{F}_{\mathbf{X}} \mathbf{F}_{\mathbf{X}}^{*}$.

We shall assume that the random variables $X_{j k}^{(q)}$, for $q=1, \ldots, m, j=1, \ldots, n_{q-1} ; k=$ $1, \ldots, n_{q}$, satisfy the following Lindeberg condition, i. e.

$$
\text { for any } \tau>0, \quad L_{n}(\tau):=\frac{1}{n^{2}} \sum_{q=1}^{m} \sum_{j=1}^{n_{q-1}} \sum_{k=1}^{n_{q}} \mathbf{E}\left|X_{j k}^{(q)}\right|^{2} \mathbb{I}\left\{\left|X_{j k}^{(q)}\right|>\tau \sqrt{n}\right\} \rightarrow 0 \quad \text { as } n \rightarrow \infty .
$$

We shall assume as well that the function $\mathbb{F}$ satisfies a so-called rank condition, i. e. for any $m$-tuples $\left(\mathbf{A}^{(1)}, \ldots, \mathbf{A}^{(m)}\right),\left(\mathbf{B}^{(1)}, \ldots, \mathbf{B}^{(m)}\right) \in \mathcal{M}_{n_{0} \times n_{1}} \times \cdots \times \mathcal{M}_{n_{m-1} \times n_{m}}$ we have

$$
\operatorname{rank}\left\{\mathbb{F}\left(\mathbf{A}^{(1)}, \ldots, \mathbf{A}^{(m)}\right)-\mathbb{F}\left(\mathbf{B}^{(1)}, \ldots, \mathbf{B}^{(m)}\right)\right\} \leq C(\mathbb{F}) \sum_{q=1}^{m} \operatorname{rank}\left\{\mathbf{A}^{(q)}-\mathbf{B}^{(q)}\right\} .
$$

We now define truncated matrices. Note that by (3.1) there exists a sequence $\left(\tau_{n}\right)$ such that

$$
\tau_{n} \rightarrow 0 \quad \text { and } \quad L_{n}\left(\tau_{n}\right) \tau_{n}^{-4} \rightarrow 0 \quad \text { as } n \rightarrow \infty .
$$

Clearly, we may additionally require that $\tau_{n} \geq n^{-1 / 3}$ for all $n$. We fix such a sequence and consider the matrix tuple $\widehat{\mathbf{X}}=\left(\widehat{\mathbf{X}}^{(1)}, \ldots, \widehat{\mathbf{X}}^{(m)}\right)$ consisting of the matrices $\widehat{\mathbf{X}}^{(q)}=$ $\left(\frac{1}{\sqrt{n_{q}}} \widehat{X}_{j k}^{(q)}\right), q=1, \ldots, m$, where

$$
\widehat{X}_{j k}^{(q)}=X_{j k}^{(q)} \mathbb{I}\left\{\left|X_{j k}^{(q)}\right| \leq \tau_{n} \sqrt{n}\right\} .
$$

Let $\mathbf{B}$ be a non-random matrix of order $n \times p$, let $\mathbf{F}_{\mathbf{X}}$ and $\mathbf{F}_{\widehat{\mathbf{X}}}$ be defined as in (2.3), and let $s_{1}(\mathbf{X}) \geq \ldots \geq s_{n}(\mathbf{X})$ and $s_{1}(\widehat{\mathbf{X}}) \geq \ldots \geq s_{n}(\widehat{\mathbf{X}})$ denote the singular values of the matrices $\mathbf{F}_{\mathbf{X}}+\mathbf{B}$ and $\mathbf{F}_{\widehat{\mathbf{X}}}+\mathbf{B}$, respectively. Let $\mathcal{F}_{\mathbf{X}}(x)$ (resp. $\mathcal{F}_{\widehat{\mathbf{X}}}(x)$ ) denote the empirical distribution function of the squared singular values of the matrix $\mathbf{F}_{\mathbf{X}}+\mathbf{B}\left(\operatorname{resp} . \mathbf{F}_{\widehat{\mathbf{X}}}+\mathbf{B}\right)$, i.e.

$$
\mathcal{F}_{\mathbf{X}}(x)=\frac{1}{n} \sum_{j=1}^{n} \mathbb{I}\left\{s_{j}^{2}(\mathbf{X}) \leq x\right\}, \quad \mathcal{F}_{\widehat{\mathbf{X}}}(x)=\frac{1}{n} \sum_{j=1}^{n} \mathbb{I}\left\{s_{j}^{2}(\widehat{\mathbf{X}}) \leq x\right\}, \quad x \in \mathbb{R} .
$$


The corresponding Stieltjes transforms of these empirical distributions are denoted by $m_{\mathbf{X}}(z)$ and $m_{\widehat{\mathbf{X}}}(z)$, i.e.

$$
m_{\mathbf{X}}(z)=\frac{1}{n} \sum_{j=1}^{n} \frac{1}{s_{j}^{2}(\mathbf{X})-z}, \quad m_{\widehat{\mathbf{X}}}(z)=\frac{1}{n} \sum_{j=1}^{n} \frac{1}{s_{j}^{2}(\widehat{\mathbf{X}})-z}, \quad z \in \mathbb{C}_{+} .
$$

First we prove the following

Lemma 3.1. Assume that the conditions (3.1) and (3.2) hold. Then

$$
\mathbf{E} \sup _{x}\left|\mathcal{F}_{\mathbf{X}}(x)-\mathcal{F}_{\widehat{\mathbf{X}}}(x)\right| \leq C \tau_{n}^{2}
$$

and, for any $z=u+i v$ with $v>0$,

$$
\mathbf{E}\left|m_{\mathbf{X}}(z)-m_{\widehat{\mathbf{X}}}(z)\right| \leq C v^{-1} \tau_{n}^{2} .
$$

Proof. By the rank inequality of Bai, see [8], Theorem A.44, we have

$$
\mathbf{E} \sup _{x}\left|\mathcal{F}_{\mathbf{X}}(x)-\mathcal{F}_{\widehat{\mathbf{X}}}(x)\right| \leq \frac{1}{n} \mathbf{E} \operatorname{rank}\left\{\mathbf{F}_{\mathbf{X}}-\mathbf{F}_{\widehat{\mathbf{X}}}\right\},
$$

and, by integration by parts,

$$
\mathbf{E}\left|m_{\mathbf{X}}(z)-m_{\widehat{\mathbf{X}}}(z)\right| \leq \frac{\pi}{n v} \mathbf{E} \operatorname{rank}\left\{\mathbf{F}_{\mathbf{X}}-\mathbf{F}_{\widehat{\mathbf{X}}}\right\} .
$$

By condition (3.2), we have

$$
\operatorname{rank}\left\{\mathbf{F}_{\mathbf{X}}-\mathbf{F}_{\widehat{\mathbf{X}}}\right\} \leq C(\mathbb{F}) \sum_{q=1}^{m} \operatorname{rank}\left\{\mathbf{X}^{(q)}-\widehat{\mathbf{X}}^{(q)}\right\}
$$

Furthermore,

$$
\begin{aligned}
\sum_{q=1}^{m} \mathbf{E} \operatorname{rank}\left\{\mathbf{X}^{(q)}-\widehat{\mathbf{X}}^{(q)}\right\} & \leq \sum_{q=1}^{m} \sum_{j=1}^{n_{q-1}} \sum_{k=1}^{n_{q}} \mathbf{E} \mathbb{I}\left\{\left|X_{j k}^{(q)}\right| \geq \tau_{n} \sqrt{n}\right\} \\
& \leq \frac{1}{n \tau_{n}^{2}} \sum_{q=1}^{m} \sum_{j=1}^{n_{q-1}} \sum_{k=1}^{n_{q}} \mathbf{E}\left|X_{j k}^{(q)}\right|^{2} \mathbb{I}\left\{\left|X_{j k}^{(q)}\right| \geq \tau_{n} \sqrt{n}\right\} \\
& =\frac{n L_{n}\left(\tau_{n}\right)}{\tau_{n}^{2}}
\end{aligned}
$$

Inequalities (3.4)-(3.7) and assumption (3.3) together complete the proof of the Lemma. 
Remark. Lemma 3.1 is about the distribution functions of the squared singular values, $\mathcal{F}_{\mathbf{X}}(x)=\frac{1}{n} \sum_{j=1}^{n} \mathbb{I}\left\{s_{j}^{2}(\mathbf{X}) \leq x\right\}(x>0)$. Similar results hold for the distribution functions of the non-squared singular values, $\mathcal{F}_{\mathbf{X}}\left(x^{2}\right)=\frac{1}{n} \sum_{j=1}^{n} \mathbb{I}\left\{s_{j}(\mathbf{X}) \leq x\right\}(x>0)$, as well as for their symmetrizations, $\widetilde{\mathcal{F}}_{\mathbf{X}}(x):=\frac{1}{2}\left(1+\operatorname{sign}(x) \mathcal{F}_{\mathbf{X}}\left(x^{2}\right)\right)(x \neq 0)$. It is this consequence of Lemma 3.1 that will be used below.

Let $\mathbf{Y}=\left(\mathbf{Y}^{(1)}, \ldots, \mathbf{Y}^{(m)}\right)$ be an $m$-tuple of independent random matrices with independent Gaussian entries as in Section $\mathbb{1}$, and let $\widehat{\mathbf{Y}}=\left(\widehat{\mathbf{Y}}^{(1)}, \ldots, \widehat{\mathbf{Y}}^{(m)}\right)$ denote the $m$-tuple consisting of the matrices $\widehat{\mathbf{Y}}^{(q)}=\left(\frac{1}{\sqrt{n_{q}}} \widehat{Y}_{j k}^{(q)}\right)$, where

$$
\widehat{Y}_{j k}^{(q)}=Y_{j k}^{(q)} \mathbb{I}\left\{\left|Y_{j k}^{(q)}\right| \leq \tau_{n} \sqrt{n}\right\} .
$$

for $q=1, \ldots, m$ and $j=1, \ldots, n_{q-1} ; k=1, \ldots, n_{q}$. Set

$$
\widetilde{L}_{n}\left(\tau_{n}\right):=\frac{1}{n^{2}} \sum_{q=1}^{m} \sum_{j=1}^{n_{q-1}} \sum_{k=1}^{n_{q}} \mathbf{E}\left|Y_{j k}^{(q)}\right|^{2} \mathbb{I}\left\{\left|Y_{j k}^{(q)}\right|>\tau_{n} \sqrt{n}\right\} .
$$

Then, using the relation $\tau_{n} \geq n^{-1 / 3}$ and the special properties of the Gaussian distribution, it is easy to check that we also have

$$
\widetilde{L}_{n}\left(\tau_{n}\right) \tau_{n}^{-4} \rightarrow 0 \quad \text { as } n \rightarrow \infty .
$$

Furthermore, note that for the truncated random variables, the moment identities (2.2) need not hold anymore. However, we have the relations

$$
\begin{gathered}
\left|\mathbf{E} \widehat{X}_{j k}^{(q)}\right|=\left|\mathbf{E} X_{j k}^{(q)} \mathbb{I}\left\{\left|X_{j k}^{(q)}\right|>\tau_{n} \sqrt{n}\right\}\right| \leq \frac{1}{\tau_{n} \sqrt{n}} \mathbf{E}\left|X_{j k}^{(q)}\right|^{2} \mathbb{I}\left\{\left|X_{j k}^{(q)}\right|>\tau_{n} \sqrt{n}\right\}, \\
\left|\mathbf{E}\left(\operatorname{Re} \widehat{X}_{j k}^{(q)}\right)^{2}-\mathbf{E}\left(\operatorname{Re} X_{j k}^{(q)}\right)^{2}\right| \leq \mathbf{E}\left|X_{j k}^{(q)}\right|^{2} \mathbb{I}\left\{\left|X_{j k}^{(q)}\right|>\tau_{n} \sqrt{n}\right\}, \\
\left|\mathbf{E}\left(\operatorname{Im} \widehat{X}_{j k}^{(q)}\right)^{2}-\mathbf{E}\left(\operatorname{Im} X_{j k}^{(q)}\right)^{2}\right| \leq \mathbf{E}\left|X_{j k}^{(q)}\right|^{2} \mathbb{I}\left\{\left|X_{j k}^{(q)}\right|>\tau_{n} \sqrt{n}\right\}, \\
\left|\mathbf{E}\left(\operatorname{Re} \widehat{X}_{j k}^{(q)} \operatorname{Im} \widehat{X}_{j k}^{(q)}\right)-\mathbf{E}\left(\operatorname{Re} X_{j k}^{(q)} \operatorname{Im} X_{j k}^{(q)}\right)\right| \leq \mathbf{E}\left|X_{j k}^{(q)}\right|^{2} \mathbb{I}\left\{\left|X_{j k}^{(q)}\right|>\tau_{n} \sqrt{n}\right\},
\end{gathered}
$$

as well as the analogous relations for the r.v.'s $\widehat{Y}_{j k}^{(q)}$, which imply that

$$
\begin{gathered}
\frac{1}{n^{3 / 2}} \sum_{q=1}^{m} \sum_{j=1}^{n_{q-1}} \sum_{k=1}^{n_{q}}\left(\left|\mathbf{E} \widehat{X}_{j k}^{(q)}\right|+\left|\mathbf{E} \widehat{Y}_{j k}^{(q)}\right|\right) \leq \frac{L\left(\tau_{n}\right)+\widetilde{L}\left(\tau_{n}\right)}{\tau_{n}}, \\
\frac{1}{n^{2}} \sum_{q=1}^{m} \sum_{j=1}^{n_{q-1}} \sum_{k=1}^{n_{q}}\left|\mathbf{E}\left(\operatorname{Re} \widehat{X}_{j k}^{(q)}\right)^{2}-\mathbf{E}\left(\operatorname{Re} \widehat{Y}_{j k}^{(q)}\right)^{2}\right| \leq L\left(\tau_{n}\right)+\widetilde{L}\left(\tau_{n}\right), \\
\frac{1}{n^{2}} \sum_{q=1}^{m} \sum_{j=1}^{n_{q-1}} \sum_{k=1}^{n_{q}}\left|\mathbf{E}\left(\operatorname{Im} \widehat{X}_{j k}^{(q)}\right)^{2}-\mathbf{E}\left(\operatorname{Im} \widehat{Y}_{j k}^{(q)}\right)^{2}\right| \leq L\left(\tau_{n}\right)+\widetilde{L}\left(\tau_{n}\right), \\
\frac{1}{n^{2}} \sum_{q=1}^{m} \sum_{j=1}^{n_{q-1}} \sum_{k=1}^{n_{q}}\left|\mathbf{E}\left(\operatorname{Re} \widehat{X}_{j k}^{(q)}\right)\left(\operatorname{Im} \widehat{X}_{j k}^{(q)}\right)-\mathbf{E}\left(\operatorname{Re} \widehat{Y}_{j k}^{(q)}\right)\left(\operatorname{Im} \widehat{Y}_{j k}^{(q)}\right)\right| \leq L\left(\tau_{n}\right)+\widetilde{L}\left(\tau_{n}\right) .
\end{gathered}
$$


For the rest of this section, we use the following notation. For any matrix tuple $\mathbf{X}=\left(\mathbf{X}^{(1)}, \ldots, \mathbf{X}^{(m)}\right)$ and any $n \times p$ matrix $\mathbf{B}$, we introduce the matrix $\mathbf{F}_{\mathbf{X}}$ as in (2.3), the Hermitian matrix

$$
\mathbf{V}_{\mathbf{X}}:=\left[\begin{array}{cc}
\mathbf{O} & \mathbf{F}_{\mathbf{X}}+\mathbf{B} \\
\left(\mathbf{F}_{\mathbf{X}}+\mathbf{B}\right)^{*} & \mathbf{O}
\end{array}\right],
$$

as well as the corresponding resolvent matrix

$$
\mathbf{R}_{\mathbf{X}}:=\mathbf{R}_{\mathbf{X}}(z)=\left(\mathbf{V}_{\mathbf{X}}-z \mathbf{I}\right)^{-1} .
$$

Furthermore, let $s_{1}(\mathbf{X}) \geq \ldots \geq s_{n}(\mathbf{X})$ denote the singular values of the matrix $\mathbf{F}_{\mathbf{X}}+\mathbf{B}$. Note that, apart from a fixed number of zero eigenvalues, the eigenvalues of the matrix $\mathbf{V}_{\mathbf{X}}$ are given by $\pm s_{1}(\mathbf{X}), \ldots \pm s_{n}(\mathbf{X})$. The corresponding Stieltjes transform will be denoted by

$$
m_{n}(z, \mathbf{X}):=\frac{1}{2 n}\left(\operatorname{Tr} \mathbf{R}_{\mathbf{X}}+\frac{p-n}{z}\right) .
$$

For $0 \leq \varphi \leq \frac{\pi}{2}$ and $q=1, \ldots, m$, let

$$
\mathbf{Z}^{(q)}(\varphi)=\left(\widehat{\mathbf{X}}^{(q)} \cos \varphi+\widehat{\mathbf{Y}}^{(q)} \sin \varphi\right)
$$

and $\mathbf{Z}(\varphi):=\left(\mathbf{Z}^{(1)}(\varphi), \ldots, \mathbf{Z}^{(m)}(\varphi)\right)$. For abbreviation, we shall write $\mathbf{F}(\varphi), \mathbf{V}(\varphi), \mathbf{R}(\varphi)$, and $m_{n}(z, \varphi)$ instead of $\mathbf{F}_{\mathbf{Z}(\varphi)}, \mathbf{V}_{\mathbf{Z}(\varphi)}, \mathbf{R}_{\mathbf{Z}(\varphi)}$, and $m_{n}(z, \mathbf{Z}(\varphi))$. With this notation we have $\mathbf{F}_{\widehat{\mathbf{X}}}=\mathbf{F}(0), \mathbf{F}_{\widehat{\mathbf{Y}}}=\mathbf{F}\left(\frac{\pi}{2}\right), m_{n}(z, \widehat{\mathbf{X}})=m_{n}(z, 0)$ and $m_{n}(z, \widehat{\mathbf{Y}})=m_{n}\left(z, \frac{\pi}{2}\right)$. Also, we may write

$$
m\left(z, \frac{\pi}{2}\right)-m_{n}(z, 0)=\int_{0}^{\frac{\pi}{2}} \frac{\partial m_{n}(z, \varphi)}{\partial \varphi} d \varphi .
$$

The representation of type (3.17) has been used for sums of random variables, for instance, in [10] (second relation on page 367). For random matrices (3.17) has been used, for example, by Pastur and Lytova in [30] (see Equation (60)).

A simple computation shows that

$$
\frac{\partial m_{n}(z, \varphi)}{\partial \varphi}=-\frac{1}{2 n} \operatorname{Tr} \frac{\partial \mathbf{V}(\varphi)}{\partial \varphi} \mathbf{R}^{2}(\varphi)
$$

Furthermore, using this relation we get

$$
\begin{aligned}
\frac{\partial m_{n}(z, \varphi)}{\partial \varphi}= & -\frac{1}{2 n} \sum_{q=1}^{m} \sum_{j=1}^{n_{q-1}} \sum_{k=1}^{n_{q}} \frac{1}{\sqrt{n_{q}}}\left(-\operatorname{Re} \widehat{X}_{j k}^{(q)} \sin \varphi+\operatorname{Re} \widehat{Y}_{j k}^{(q)} \cos \varphi\right) \operatorname{Tr} \frac{\partial \mathbf{V}}{\partial \operatorname{Re} Z_{j k}^{(q)}} \mathbf{R}^{2} \\
& -\frac{i}{2 n} \sum_{q=1}^{m} \sum_{j=1}^{n_{q-1}} \sum_{k=1}^{n_{q}} \frac{1}{\sqrt{n_{q}}}\left(-\operatorname{Im} \widehat{X}_{j k}^{(q)} \sin \varphi+\operatorname{Im} \widehat{Y}_{j k}^{(q)} \cos \varphi\right) \operatorname{Tr} \frac{\partial \mathbf{V}}{\partial \operatorname{Im} Z_{j k}^{(q)}} \mathbf{R}^{2} .
\end{aligned}
$$


We denote by

$$
\begin{aligned}
& g_{j k}^{(q)}:=g_{j k}^{(q)}\left(\mathbf{Z}^{(1)}(\varphi), \ldots, \mathbf{Z}^{(m)}(\varphi)\right)=\operatorname{Tr} \frac{\partial \mathbf{V}}{\partial \operatorname{Re} Z_{j k}^{(q)}} \mathbf{R}^{2} \\
& \widehat{g}_{j k}^{(q)}:=\widehat{g}_{j k}^{(q)}\left(\mathbf{Z}^{(1)}(\varphi), \ldots, \mathbf{Z}^{(m)}(\varphi)\right)=\operatorname{Tr} \frac{\partial \mathbf{V}}{\partial \operatorname{Im} Z_{j k}^{(q)}} \mathbf{R}^{2}
\end{aligned}
$$

Let $g_{j k}^{(q)}(\theta)$ denote the function obtained from $g_{j k}^{(q)}$ by replacing the indeterminate $Z_{j k}^{(q)}$ with $\theta Z_{j k}^{(q)}$.

Theorem 3.2. Assume that the Lindeberg condition (3.1) and the rank condition (3.2) hold. Furthermore suppose that there exist constants $A_{0}>0, A_{1}>0$ and $A_{2}>0$ such that for any random variable $\theta$ which is uniformly distributed on the interval $[0,1]$ and independent of the r.v.'s $X_{j k}^{(q)}$ and $Y_{j k}^{(q)}$, the following conditions hold:

$$
\begin{aligned}
& \sup _{j, k, q}\left\|\mathbf{E}\left\{g_{j k}^{(q)}(\theta) \mid X_{j k}^{(q)}, Y_{j k}^{(q)}\right\}\right\|_{\infty} \leq A_{0}, \\
& \sup _{j, k, q}\left\|\mathbf{E}\left\{\widehat{g}_{j k}^{(q)}(\theta) \mid X_{j k}^{(q)}, Y_{j k}^{(q)}\right\}\right\|_{\infty} \leq A_{0}, \\
& \sup _{j, k, q} \max \left\{\left\|\mathbf{E}\left\{\frac{\partial g_{j k}^{(q)}(\theta)}{\partial \operatorname{Re} Z_{j k}^{(q)}} \mid X_{j k}^{(q)}, Y_{j k}^{(q)}\right\}\right\|_{\infty},\left\|\mathbf{E}\left\{\frac{\partial g_{j k}^{(q)}(\theta)}{\partial \operatorname{Im} Z_{j k}^{(q)}} \mid X_{j k}^{(q)}, Y_{j k}^{(q)}\right\}\right\|_{\infty}\right\} \leq A_{1}, \\
& \sup _{j, k, q} \max \left\{\left\|\mathbf{E}\left\{\frac{\partial \widehat{g}_{j k}^{(q)}(\theta)}{\partial \operatorname{Re} Z_{j k}^{(q)}} \mid X_{j k}^{(q)}, Y_{j k}^{(q)}\right\}\right\|_{\infty},\left\|\mathbf{E}\left\{\frac{\partial \widehat{g}_{j k}^{(q)}(\theta)}{\partial \operatorname{Im} Z_{j k}^{(q)}} \mid X_{j k}^{(q)}, Y_{j k}^{(q)}\right\}\right\|_{\infty}\right\} \leq A_{1}, \\
& \sup _{j, k, q} \max \left\{\left\|\mathbf{E}\left\{\frac{\partial^{2} g_{j k}^{(q)}(\theta)}{\partial \operatorname{Re} Z_{j k}^{(q)}} \mid X_{j k}^{(q)}, Y_{j k}^{(q)}\right\}\right\|_{\infty},\left\|\mathbf{E}\left\{\frac{\partial^{2} g_{j k}^{(q)}(\theta)}{\partial \operatorname{Im} Z_{j k}^{(q)}} \mid X_{j k}^{(q)}, Y_{j k}^{(q)}\right\}\right\|_{\infty},\right. \\
& \left.\qquad \mathbf{E}\left\{\frac{\partial^{2} g_{j k}^{(q)}(\theta)}{\partial \operatorname{Re} Z_{j k}^{(q)} \partial \operatorname{Im} Z_{j k}^{(q)}} \mid X_{j k}^{(q)}, Y_{j k}^{(q)}\right\} \|_{\infty}\right\} \leq A_{2}, \\
& \sup _{j, k, q} \max \left\{\left\|\mathbf{E}\left\{\frac{\partial^{2} \widehat{g}_{j k}^{(q)}(\theta)}{\partial \operatorname{Re} Z_{j k}^{(q)}} \mid X_{j k}^{(q)}, Y_{j k}^{(q)}\right\}\right\|_{\infty},\left\|\mathbf{E}\left\{\frac{\partial^{2} \widehat{g}_{j k}^{(q)}(\theta)}{\partial \operatorname{Im} Z_{j k}^{(q)}} \mid X_{j k}^{(q)}, Y_{j k}^{(q)}\right\}\right\|_{\infty},\right. \\
& \left.\partial \mathbf{E}\left\{\frac{\partial^{2} \widehat{g}_{j k}^{(q)}(\theta)}{\partial \operatorname{Re} Z_{j k}^{(q)} \partial \operatorname{Im} Z_{j k}^{(q)}} \mid X_{j k}^{(q)}, Y_{j k}^{(q)}\right\} \|_{\infty}\right\} \leq A_{2} .
\end{aligned}
$$

Then, for any $z=u+i v$ with $v>0$,

$$
\lim _{n \rightarrow \infty}\left(m_{n}(z, \mathbf{Y})-m_{n}(z, \mathbf{X})\right)=0 \quad \text { in probability }
$$


Remark. It follows from the conclusion of the theorem and basic properties of the Stieltjes transform that if the singular value distributions of the matrices $\mathbf{F}_{\mathbf{Y}}+\mathbf{B}$ are weakly convergent in probability to some limit $\nu$, then so are the singular value distributions of the matrices $\mathbf{F}_{\mathbf{X}}+\mathbf{B}$. In this sense Theorem 3.2 proves the universality of singular value distributions.

Proof of Theorem 3.2. By Lemma 3.1 and the subsequent remark, it is sufficient to prove the claim with $m_{n}(z, \widehat{\mathbf{X}})$ and $m_{n}(z, \widehat{\mathbf{Y}})$ instead of $m_{n}(z, \mathbf{X})$ and $m_{n}(z, \mathbf{Y})$. Furthermore, according to Lemma A.1 in the Appendix, it is enough to prove that

$$
\lim _{n \rightarrow \infty} \mathbf{E}\left(m\left(z, \frac{\pi}{2}\right)-m_{n}(z, 0)\right)=0 .
$$

Using Taylor's formula in the form

$$
\begin{aligned}
f(x, y)=f(0,0)+x f_{x}^{\prime}(0,0) & +y f_{y}^{\prime}(0,0)+x^{2} \mathbf{E}_{\theta}(1-\theta) f_{x x}^{\prime \prime}(\theta x, \theta y) \\
& +2 x y \mathbf{E}_{\theta}(1-\theta) f_{x y}^{\prime \prime}(\theta x, \theta y)+y^{2} \mathbf{E}_{\theta}(1-\theta) f_{y y}^{\prime \prime}(\theta x, \theta y),
\end{aligned}
$$

where $\theta$ is a random variable which is uniformly distributed on the unit interval, we get

$$
\begin{aligned}
g_{j k}^{(q)} & =g_{j k}^{(q)}(0)+\frac{1}{\sqrt{n_{q}}}\left(\operatorname{Re} \widehat{X}_{j k}^{(q)} \cos \varphi+\operatorname{Re} \widehat{Y}_{j k}^{(q)} \sin \varphi\right) \frac{\partial g_{j k}^{(q)}}{\partial \operatorname{Re} Z_{j k}^{(q)}}(0) \\
& +\frac{1}{\sqrt{n_{q}}}\left(\operatorname{Im} \widehat{X}_{j k}^{(q)} \cos \varphi+\operatorname{Im} \widehat{Y}_{j k}^{(q)} \sin \varphi\right) \frac{\partial g_{j k}^{(q)}}{\partial \operatorname{Im} Z_{j k}^{(q)}}(0) \\
& +\frac{1}{n_{q}}\left(\operatorname{Re} \widehat{X}_{j k}^{(q)} \cos \varphi+\operatorname{Re} \widehat{Y}_{j k}^{(q)} \sin \varphi\right)^{2} \mathbf{E}_{\theta}(1-\theta) \frac{\partial^{2} g_{j k}^{(q)}}{\partial \operatorname{Re} Z_{j k}^{(q)^{2}}}(\theta) \\
& +\frac{2}{n_{q}}\left(\operatorname{Re} \widehat{X}_{j k}^{(q)} \cos \varphi+\operatorname{Re} \widehat{Y}_{j k}^{(q)} \sin \varphi\right)\left(\operatorname{Im} \widehat{X}_{j k}^{(q)} \cos \varphi+\operatorname{Im} \widehat{Y}_{j k}^{(q)} \sin \varphi\right) \\
& \times \mathbf{E}_{\theta}(1-\theta) \frac{\partial^{2} g_{j k}^{(q)}}{\partial \operatorname{Re} Z_{j k}^{(q)} \partial \operatorname{Im} Z_{j k}^{(q)}}(\theta) \\
& +\frac{1}{n_{q}}\left(\operatorname{Im} \widehat{X}_{j k}^{(q)} \cos \varphi+\operatorname{Im} \widehat{Y}_{j k}^{(q)} \sin \varphi\right)^{2} \mathbf{E}_{\theta}(1-\theta) \frac{\partial^{2} g_{j k}^{(q)}}{\partial \operatorname{Im} Z_{j k}^{(q)}}(\theta) .
\end{aligned}
$$

Here $\mathbf{E}_{\theta}$ denotes the expectation with respect to the r.v. $\theta$ conditioning on all other r.v.'s. Inserting (3.28) into (3.18), we get

$$
\mathbf{E}\left(m\left(z, \frac{\pi}{2}\right)-m_{n}(z, 0)\right)=-\sum_{j=1}^{12} \Sigma_{j}
$$


where

$$
\begin{aligned}
& \Sigma_{1}=\frac{1}{2 n} \sum_{q=1}^{m} \sum_{j=1}^{n_{q-1}} \sum_{k=1}^{n_{q}} \int_{0}^{\frac{\pi}{2}} \frac{1}{\sqrt{n_{q}}} \mathbf{E}\left(\left(-\operatorname{Re} \widehat{X}_{j k}^{(q)} \sin \varphi+\operatorname{Re} \widehat{Y}_{j k}^{(q)} \cos \varphi\right) g_{j k}^{(q)}(0,0)\right) d \varphi, \\
& \Sigma_{2}=\frac{1}{2 n} \sum_{q=1}^{m} \sum_{j=1}^{n_{q-1}} \sum_{k=1}^{n_{q}} \int_{0}^{\frac{\pi}{2}} \frac{1}{n_{q}} \mathbf{E}\left(\left(-\operatorname{Re} \widehat{X}_{j k}^{(q)} \sin \varphi+\operatorname{Re} \widehat{Y}_{j k}^{(q)} \cos \varphi\right)\right. \\
& \left.\times\left(\operatorname{Re} \widehat{X}_{j k}^{(q)} \cos \varphi+\operatorname{Re} \widehat{Y}_{j k}^{(q)} \sin \varphi\right) \frac{\partial g_{j k}^{(q)}}{\partial \operatorname{Re} Z_{j k}^{(q)}}(0,0)\right) d \varphi, \\
& \Sigma_{3}=\frac{1}{2 n} \sum_{q=1}^{m} \sum_{j=1}^{n_{q-1}} \sum_{k=1}^{n_{q}} \int_{0}^{\frac{\pi}{2}} \frac{1}{n_{q}} \mathbf{E}\left(\left(-\operatorname{Re} \widehat{X}_{j k}^{(q)} \sin \varphi+\operatorname{Re} \widehat{Y}_{j k}^{(q)} \cos \varphi\right)\right. \\
& \left.\times\left(\operatorname{Im} \widehat{X}_{j k}^{(q)} \cos \varphi+\operatorname{Im} \widehat{Y}_{j k}^{(q)} \sin \varphi\right) \frac{\partial g_{j k}^{(q)}}{\partial \operatorname{Im} Z_{j k}^{(q)}}(0,0)\right) d \varphi, \\
& \Sigma_{4}=\frac{1}{2 n} \sum_{q=1}^{m} \sum_{j=1}^{n_{q-1}} \sum_{k=1}^{n_{q}} \int_{0}^{\frac{\pi}{2}} \frac{1}{n_{q} \sqrt{n_{q}}} \mathbf{E}\left(\left(-\operatorname{Re} \widehat{X}_{j k}^{(q)} \sin \varphi+\operatorname{Re} \widehat{Y}_{j k}^{(q)} \cos \varphi\right)\right. \\
& \left.\times\left(\operatorname{Re} \widehat{X}_{j k}^{(q)} \cos \varphi+\operatorname{Re} \widehat{Y}_{j k}^{(q)} \sin \varphi\right)^{2}\left(1-\theta_{j k}^{(q)}\right) \frac{\partial^{2} g_{j k}^{(q)}}{\partial \operatorname{Re} Z_{j k}^{(q)^{2}}}\left(\theta_{j k}^{(q)}\right)\right) d \varphi, \\
& \Sigma_{5}=\frac{1}{2 n} \sum_{q=1}^{m} \sum_{j=1}^{n_{q-1}} \sum_{k=1}^{n_{q}} \int_{0}^{\frac{\pi}{2}} \frac{1}{n_{q \sqrt{n_{q}}}} \mathbf{E}\left(\left(-\operatorname{Re} \widehat{X}_{j k}^{(q)} \sin \varphi+\operatorname{Re} \widehat{Y}_{j k}^{(q)} \cos \varphi\right)\right. \\
& \left.\times\left(\operatorname{Im} \widehat{X}_{j k}^{(q)} \cos \varphi+\operatorname{Im} \widehat{Y}_{j k}^{(q)} \sin \varphi\right)^{2}\left(1-\theta_{j k}^{(q)}\right) \frac{\partial^{2} g_{j k}^{(q)}}{\partial \operatorname{Im} Z_{j k}^{(q)^{2}}}\left(\theta_{j k}^{(q)}\right)\right) d \varphi, \\
& \Sigma_{6}=\frac{1}{n} \sum_{q=1}^{m} \sum_{j=1}^{n_{q-1}} \sum_{k=1}^{n_{q}} \int_{0}^{\frac{\pi}{2}} \frac{1}{n_{q} \sqrt{n_{q}}} \mathbf{E}\left(\left(-\operatorname{Re} \widehat{X}_{j k}^{(q)} \sin \varphi+\operatorname{Re} \widehat{Y}_{j k}^{(q)} \cos \varphi\right)\right. \\
& \times\left(\operatorname{Im} \widehat{X}_{j k}^{(q)} \cos \varphi+\operatorname{Im} \widehat{Y}_{j k}^{(q)} \sin \varphi\right)\left(\operatorname{Re} \widehat{X}_{j k}^{(q)} \cos \varphi+\operatorname{Re} \widehat{Y}_{j k}^{(q)} \sin \varphi\right) \\
& \left.\times\left(1-\theta_{j k}^{(q)}\right) \frac{\partial^{2} g_{j k}^{(q)}}{\partial \operatorname{Im} Z_{j k}^{(q)} \partial \operatorname{Re} Z_{j k}^{(q)}}\left(\theta_{j k}^{(q)}\right)\right) d \varphi,
\end{aligned}
$$

and $\Sigma_{7}, \ldots, \Sigma_{12}$ denote similar terms coming from the second line in (3.18). Since $\Sigma_{7}, \ldots, \Sigma_{12}$ can be treated in the same way as $\Sigma_{1}, \ldots, \Sigma_{6}$, we provide the details for the latter only.

Since $g_{j k}^{(q)}(0,0)$ and $X_{j k}^{(q)}, Y_{j k}^{(q)}$ are independent, it follows from (3.13) that

$$
\left|\Sigma_{1}\right| \leq \frac{C A_{0}\left(L_{n}\left(\tau_{n}\right)+\widetilde{L}_{n}\left(\tau_{n}\right)\right)}{\tau_{n}} \leq C \tau_{n}^{3}
$$


Using again that the random variables $\frac{\partial g_{j k}^{(q)}}{\partial Z_{j k}^{(q)}}(0,0)$ and $X_{j k}^{(q)}, Y_{j k}^{(q)}$ are independent, we get

$$
\begin{aligned}
& \mathbf{E}\left(\left(-\operatorname{Re} \widehat{X}_{j k}^{(q)} \sin \varphi+\operatorname{Re} \widehat{Y}_{j k}^{(q)} \cos \varphi\right)\left(\operatorname{Re} \widehat{X}_{j k}^{(q)} \cos \varphi+\operatorname{Re} \widehat{Y}_{j k}^{(q)} \sin \varphi\right) \frac{\partial g_{j k}^{(q)}}{\partial \operatorname{Re} Z_{j k}^{(q)}}(0,0)\right) \\
= & \mathbf{E}\left(\left(-\operatorname{Re} \widehat{X}_{j k}^{(q)} \sin \varphi+\operatorname{Re} \widehat{Y}_{j k}^{(q)} \cos \varphi\right)\left(\operatorname{Re} \widehat{X}_{j k}^{(q)} \cos \varphi+\operatorname{Re} \widehat{Y}_{j k}^{(q)} \sin \varphi\right)\right) \mathbf{E}\left(\frac{\partial g_{j k}^{(q)}}{\partial \operatorname{Re} Z_{j k}^{(q)}}(0,0)\right) .
\end{aligned}
$$

Since $\operatorname{Re} \widehat{X}_{j k}^{(q)}$ and $\operatorname{Re} \widehat{Y}_{j k}^{(q)}$ are independent, we have

$$
\begin{aligned}
\mathbf{E} & \left(-\operatorname{Re} \widehat{X}_{j k}^{(q)} \sin \varphi+\operatorname{Re} \widehat{Y}_{j k}^{(q)} \cos \varphi\right)\left(\operatorname{Re} \widehat{X}_{j k}^{(q)} \cos \varphi+\operatorname{Re} \widehat{Y}_{j k}^{(q)} \sin \varphi\right) \\
& =\mathbf{E} \operatorname{Re} \widehat{X}_{j k}^{(q)} \mathbf{E} \operatorname{Re} \widehat{Y}_{j k}^{(q)}\left(\cos ^{2} \varphi-\sin ^{2} \varphi\right)-\left(\mathbf{E}\left|\operatorname{Re} \widehat{X}_{j k}^{(q)}\right|^{2}-\mathbf{E}\left|\operatorname{Re} \widehat{Y}_{j k}^{(q)}\right|^{2}\right) \cos \varphi \sin \varphi
\end{aligned}
$$

and therefore, by (3.9),

$$
\begin{aligned}
& \left|\mathbf{E}\left(-\operatorname{Re} \widehat{X}_{j k}^{(q)} \sin \varphi+\operatorname{Re} \widehat{Y}_{j k}^{(q)} \cos \varphi\right)\left(\operatorname{Re} \widehat{X}_{j k}^{(q)} \cos \varphi+\operatorname{Re} \widehat{Y}_{j k}^{(q)} \sin \varphi\right)\right| \\
& \leq\left|\mathbf{E} \operatorname{Re} \widehat{X}_{j k}^{(q)}\right|^{2}+\left|\mathbf{E} \operatorname{Re} \widehat{Y}_{j k}^{(q)}\right|^{2}+\left.|\mathbf{E}| \operatorname{Re} \widehat{X}_{j k}^{(q)}\right|^{2}-\mathbf{E}\left|\operatorname{Re} \widehat{Y}_{j k}^{(q)}\right|^{2} \mid \\
& \leq \frac{1}{n \tau_{n}^{2}}\left(\mathbf{E}\left|X_{j k}^{(q)}\right|^{2} \mathbb{I}\left\{\left|X_{j k}^{(q)}\right| \geq \tau_{n} \sqrt{n}\right\}\right)^{2}+\frac{1}{n \tau_{n}^{2}}\left(\mathbf{E}\left|Y_{j k}^{(q)}\right|^{2} \mathbb{I}\left\{\left|Y_{j k}^{(q)}\right| \geq \tau_{n} \sqrt{n}\right\}\right)^{2} \\
& \quad+\left.|\mathbf{E}| \operatorname{Re} \widehat{X}_{j k}^{(q)}\right|^{2}-\mathbf{E}\left|\operatorname{Re} \widehat{Y}_{j k}^{(q)}\right|^{2} \mid \\
& \leq \frac{1}{n \tau_{n}^{2}} \mathbf{E}\left|X_{j k}^{(q)}\right|^{2} \mathbb{I}\left\{\left|X_{j k}^{(q)}\right| \geq \tau_{n} \sqrt{n}\right\}+\frac{1}{n \tau_{n}^{2}} \mathbf{E}\left|Y_{j k}^{(q)}\right|^{2} \mathbb{I}\left\{\left|Y_{j k}^{(q)}\right| \geq \tau_{n} \sqrt{n}\right\} \\
& \quad+\left.|\mathbf{E}| \operatorname{Re} \widehat{X}_{j k}^{(q)}\right|^{2}-\mathbf{E}\left|\operatorname{Re} \widehat{Y}_{j k}^{(q)}\right|^{2} \mid
\end{aligned}
$$

By (3.14), the last inequality implies that

$$
\left|\Sigma_{2}\right| \leq C A_{1}\left(\frac{L_{n}\left(\tau_{n}\right)+\widetilde{L}_{n}\left(\tau_{n}\right)}{n \tau_{n}^{2}}+L_{n}\left(\tau_{n}\right)+\widetilde{L}_{n}\left(\tau_{n}\right)\right) \leq C\left(\frac{\tau_{n}^{2}}{n}+\tau_{n}^{4}\right) .
$$

Similarly, using (3.9) and (3.16), we get

$$
\left|\Sigma_{3}\right| \leq C A_{1}\left(\frac{L_{n}\left(\tau_{n}\right)+\widetilde{L}_{n}\left(\tau_{n}\right)}{n \tau_{n}^{2}}+L_{n}\left(\tau_{n}\right)+\widetilde{L}_{n}\left(\tau_{n}\right)\right) \leq C\left(\frac{\tau_{n}^{2}}{n}+\tau_{n}^{4}\right) .
$$


Also, note that

$$
\begin{aligned}
\frac{1}{\sqrt{n}} \mid \mathbf{E}((- & \left.\operatorname{Re} \widehat{X}_{j k}^{(q)} \sin \varphi+\operatorname{Re} \widehat{Y}_{j k}^{(q)} \cos \varphi\right)\left(\operatorname{Re} \widehat{X}_{j k}^{(q)} \cos \varphi+\operatorname{Re} \widehat{Y}_{j k}^{(q)} \sin \varphi\right)^{2} \\
& \left.\times\left(1-\theta_{j k}^{(q)}\right) \frac{\partial^{2} g_{j k}^{(q)}}{\partial \operatorname{Re} Z_{j k}^{(q)^{2}}}\left(\theta_{j k}^{(q)}\right)\right) \mid \\
\leq & \frac{C}{\sqrt{n}} \mathbf{E}\left(\left(\left|\widehat{X}_{j k}^{(q)}\right|^{3}+\left|\widehat{Y}_{j k}^{(q)}\right|^{3}\right)\left|\mathbf{E}\left\{\frac{\partial^{2} g_{j k}^{(q)}}{\partial \operatorname{Re} Z_{j k}^{(q)}}\left(\theta_{j k}^{(q)}\right) \mid \widehat{X}_{j k}^{(q)}, \widehat{Y}_{j k}^{(q)}\right\}\right|\right) \\
\leq & \frac{C A_{2}}{\sqrt{n}} \mathbf{E}\left(\left|\widehat{X}_{j k}^{(q)}\right|^{3}+\left|\widehat{Y}_{j k}^{(q)}\right|^{3}\right) \leq C A_{2} \tau_{n} .
\end{aligned}
$$

It is simple to check now that

$$
\left|\Sigma_{4}\right| \leq C A_{2} \tau_{n}
$$

Analogously we show that

$$
\max \left\{\left|\Sigma_{5}\right|,\left|\Sigma_{6}\right|\right\} \leq C A_{2} \tau_{n} .
$$

Combining the preceding estimates, we obtain (3.26). Thus, Theorem 3.2 is proved.

\section{Universality of Eigenvalue Distributions of Functions of Independent Random Matrices}

We now turn to the eigenvalue distribution of functions of independent random matrices. We use the assumptions and the notation from Section 1, but throughout this section we assume additionally that $n=p$, so that $\mathbf{F}_{\mathbf{X}}$ and $\mathbf{F}_{\mathbf{Y}}$ are square matrices.

Let $\mu$ a probability measure on the complex plane with compact support. Define the logarithmic potential of the measure $\mu$ as

$$
U_{\mu}(\alpha)=-\int_{\mathbb{C}} \log |\alpha-\zeta| d \mu(\zeta)
$$

Let $\mu_{\mathbf{X}}\left(\right.$ resp. $\left.\mu_{\mathbf{Y}}\right)$ denote the empirical spectral measure of the matrix $\mathbf{F}_{\mathbf{X}}\left(\operatorname{resp} . \mathbf{F}_{\mathbf{Y}}\right)$, i.e. $\mu_{\mathbf{X}}\left(\right.$ resp. $\left.\mu_{\mathbf{Y}}\right)$ is the uniform distribution on the eigenvalues $\left\{\lambda_{1}(\mathbf{X}), \ldots, \lambda_{n}(\mathbf{X})\right\}$ (resp. $\left.\left\{\lambda_{1}(\mathbf{Y}), \ldots \lambda_{n}(\mathbf{Y})\right\}\right)$ of the matrix $\mathbf{F}_{\mathbf{X}}\left(\operatorname{resp} . \mathbf{F}_{\mathbf{Y}}\right)$. Then

$$
\begin{aligned}
& U_{\mathbf{X}}(\alpha)=-\int_{\mathbb{C}} \log |\alpha-\zeta| d \mu_{\mathbf{X}}(\zeta)=-\frac{1}{n} \sum_{j=1}^{n} \log \left|\lambda_{j}(\mathbf{X})-\alpha\right|, \\
& U_{\mathbf{Y}}(\alpha)=-\int_{\mathbb{C}} \log |\alpha-\zeta| d \mu_{\mathbf{Y}}(\zeta)=-\frac{1}{n} \sum_{j=1}^{n} \log \left|\lambda_{j}(\mathbf{Y})-\alpha\right| .
\end{aligned}
$$


Let $\alpha \in \mathbb{C}$, and let $s_{1}\left(\mathbf{F}_{\mathbf{X}}-\alpha \mathbf{I}\right) \geq \cdots \geq s_{n}\left(\mathbf{F}_{\mathbf{X}}-\alpha \mathbf{I}\right)$ and $s_{1}\left(\mathbf{F}_{\mathbf{Y}}-\alpha \mathbf{I}\right) \geq \cdots \geq$ $s_{n}\left(\mathbf{F}_{\mathbf{Y}}-\alpha \mathbf{I}\right)$ denote the singular values of the matrices $\mathbf{F}_{\mathbf{X}}-\alpha \mathbf{I}$ and $\mathbf{F}_{\mathbf{Y}}-\alpha \mathbf{I}$, respectively. Note that we have the representations

$$
U_{\mathbf{X}}(\alpha)=-\frac{1}{n} \sum_{j=1}^{n} \log s_{j}\left(\mathbf{F}_{\mathbf{X}}-\alpha \mathbf{I}\right), \quad U_{\mathbf{Y}}(\alpha)=-\frac{1}{n} \sum_{j=1}^{n} \log s_{j}\left(\mathbf{F}_{\mathbf{Y}}-\alpha \mathbf{I}\right) .
$$

Definition 4.1. If there exists some $p>0$ such that the quantity

$$
\frac{1}{n} \sum_{k=1}^{n} s_{k}^{p}\left(\mathbf{F}_{\mathbf{X}}\right)
$$

is bounded in probability as $n \rightarrow \infty$, we say that the matrices $\mathbf{F}_{\mathbf{X}}$ satisfy condition $(C 0)$.

Definition 4.2. If, for any fixed $\alpha \in \mathbb{C}$, there exists some $Q>0$ such that the relation

$$
\lim _{n \rightarrow \infty} \operatorname{Pr}\left\{s_{n}\left(\mathbf{F}_{\mathbf{X}}-\alpha \mathbf{I}\right) \leq n^{-Q}\right\}=0
$$

holds, we say that the matrices $\mathbf{F}_{\mathbf{X}}$ satisfy condition $(C 1)$.

Definition 4.3. If, for any fixed $\alpha \in \mathbb{C}$, there exists some $0<\gamma<1$ such that for any sequence $\delta_{n} \rightarrow 0$,

$$
\lim _{n \rightarrow \infty} \operatorname{Pr}\left\{\frac{1}{n} \sum_{n_{1} \leq j \leq n_{2}}\left|\log s_{j}\left(\mathbf{F}_{\mathbf{X}}-\alpha \mathbf{I}\right)\right|>\varepsilon\right\}=0 \quad \text { for all } \varepsilon>0,
$$

with $n_{1}=\left[n-n \delta_{n}\right]+1, n_{2}=\left[n-n^{\gamma}\right]$, we say that the matrices $\mathbf{F}_{\mathbf{X}}$ satisfy condition $(C 2)$.

We now prove the universality of eigenvalue distributions.

Theorem 4.4. Assume that the matrices $\mathbf{F}_{\mathbf{X}}$ and $\mathbf{F}_{\mathbf{Y}}$ satisfy the conditions $(C 0),(C 1)$ and (C2). Assume additionally that the conditions (3.1) and (3.2) hold and that, for any fixed $\alpha \in \mathbb{C}$, the conditions (3.20) - (3.25) of Theorem 3.2 hold with $\mathbf{B}=\alpha \mathbf{I}$. Then the empirical distributions of the eigenvalues of the matrices $\mathbf{F}_{\mathbf{X}}$ and $\mathbf{F}_{\mathbf{Y}}$ have the same limit distribution in probability in the sense that

$$
\lim _{n \rightarrow \infty} \operatorname{Pr}\left\{\left|\int_{\mathbb{C}} f d \mu_{\mathbf{X}}-\int_{\mathbb{C}} f d \mu_{\mathbf{Y}}\right|>\varepsilon\right\}=0
$$

for any bounded continuous function $f$ and any $\varepsilon>0$.

Proof. The proof of Theorem 4.4 is based on the "replacement principle" by Tao and Vu (see [41], Theorem 2.1) which builds upon a sort of inversion formula for the logarithmic potential that goes back to Girko [22] and that was also investigated by Bai [7], 8].

We prove that, for any fixed $\alpha \in \mathbb{C}$,

$$
\lim _{n \rightarrow \infty}\left(\frac{1}{n} \log \left|\operatorname{det}\left\{\mathbf{F}_{\mathbf{X}}-\alpha \mathbf{I}\right\}\right|-\frac{1}{n} \log \left|\operatorname{det}\left\{\mathbf{F}_{\mathbf{Y}}-\alpha \mathbf{I}\right\}\right|\right)=0 \quad \text { in probability. }
$$


This is equivalent to

$$
\lim _{n \rightarrow \infty}\left(U_{\mathbf{X}}(\alpha)-U_{\mathbf{Y}}(\alpha)\right)=0 \quad \text { in probability. }
$$

Note that by condition (C1) the determinants in (4.3) are not zero with probability $1-o(1)$.

Let $L(F, G)$ denote the Lévy distance between two distribution functions $F$ and $G$. Recall that

$$
L(F, G)=\inf \{\varepsilon>0: F(x-\varepsilon)-\varepsilon \leq G(x) \leq F(x+\varepsilon)+\varepsilon \text { for all } x \in \mathbb{R}\} \in[0,1] .
$$

Let $\mathcal{G}_{\mathbf{X}}(x, \alpha)$ and $\mathcal{G}_{\mathbf{Y}}(x, \alpha)$ denote the distribution functions of the singular values of the matrices $\mathbf{F}_{\mathbf{X}}-\alpha \mathbf{I}$ and $\mathbf{F}_{\mathbf{Y}}-\alpha \mathbf{I}$, respectively. Let $\varkappa_{n}=L\left(\mathcal{G}_{\mathbf{X}}(\cdot, \alpha), \mathcal{G}_{\mathbf{Y}}(\cdot, \alpha)\right)$. According to Theorem 3.2 (with $\mathbf{B}=\alpha \mathbf{I}$ ), we have

$$
\lim _{n \rightarrow \infty} \varkappa_{n}=0 \quad \text { in probability. }
$$

We introduce the events

$$
\Omega_{0}=\left\{s_{n}\left(\mathbf{F}_{\mathbf{X}}-\alpha \mathbf{I}\right) \geq n^{-Q}\right\} \cap\left\{s_{n}\left(\mathbf{F}_{\mathbf{Y}}-\alpha \mathbf{I}\right) \geq n^{-Q}\right\}, \quad \Omega_{1}=\left\{\varkappa_{n} \leq\left(\mathbf{E} \varkappa_{n}\right)^{\frac{1}{2}}\right\} .
$$

Note that by (4.4) and Markov's inequality, we have

$$
\operatorname{Pr}\left\{\Omega_{1}^{c}\right\} \leq\left(\mathbf{E} \varkappa_{n}\right)^{\frac{1}{2}} \rightarrow 0 \text { as } n \rightarrow \infty
$$

Furthermore, put $\eta_{n}=\max \left\{\left(\mathbf{E} \varkappa_{n}\right)^{\frac{1}{3}},(\log n)^{-1}\right\}$, and introduce the event

$$
\Omega_{2}=\left\{\text { there exists } a \in\left[\eta_{n}, 2 \eta_{n}\right]: \mathcal{G}_{\mathbf{Y}}\left(a+\varkappa_{n}, \alpha\right)-\mathcal{G}_{\mathbf{Y}}\left(a-\varkappa_{n}, \alpha\right) \leq \varkappa_{n}^{\frac{1}{2}}\right\} .
$$

It is straightforward to check that

$$
\operatorname{Pr}\left\{\Omega_{2}^{c}\right\} \leq \operatorname{Pr}\left\{\mathcal{G}_{\mathbf{Y}}\left(2 \eta_{n}, \alpha\right)-\mathcal{G}_{\mathbf{Y}}\left(\eta_{n}, \alpha\right) \geq \varkappa_{n}^{\frac{1}{2}}\left[\frac{\eta_{n}}{2 \varkappa_{n}}\right]\right\} \leq \operatorname{Pr}\left\{\frac{\eta_{n}}{2 \varkappa_{n}^{\frac{1}{2}}} \leq 2\right\} \leq \frac{16 \mathbf{E} \varkappa_{n}}{\eta_{n}^{2}} \leq 16 \eta_{n}
$$

Let $\delta_{n}:=1 /\left|\log 2 \eta_{n}\right| \rightarrow 0$, let $n_{1}=\left[n-n \delta_{n}\right]+1$ and $n_{2}=\left[n-n^{\gamma}\right]$ be defined as in condition (C2), and introduce the event

$$
\Omega_{3}=\left\{s_{n_{1}}\left(\mathbf{F}_{\mathbf{X}}-\alpha \mathbf{I}\right) \geq 2 \eta_{n}\right\} \cap\left\{s_{n_{1}}\left(\mathbf{F}_{\mathbf{Y}}-\alpha \mathbf{I}\right) \geq 2 \eta_{n}\right\} .
$$

Note that on the set $\left\{s_{n_{1}}\left(\mathbf{F}_{\mathbf{X}}-\alpha \mathbf{I}\right)<2 \eta_{n}\right\}$, we have, for large enough $n$,

$$
\begin{array}{r}
\frac{1}{n} \sum_{n_{1} \leq j \leq n_{2}}\left|\log s_{j}\left(\mathbf{F}_{\mathbf{X}}-\alpha \mathbf{I}\right)\right| \geq \frac{1}{n}\left(n_{2}-n_{1}+1\right)\left|\log 2 \eta_{n}\right| \geq \frac{1}{n}\left(n \delta_{n}-n^{\gamma}-1\right)\left|\log 2 \eta_{n}\right| \\
=\left(\delta_{n}-n^{\gamma-1}-n^{-1}\right)\left|\log 2 \eta_{n}\right| \geq \frac{1}{2} \delta_{n}\left|\log 2 \eta_{n}\right|=\frac{1}{2},
\end{array}
$$

so that

$$
\operatorname{Pr}\left\{s_{n_{1}}\left(\mathbf{F}_{\mathbf{X}}-\alpha \mathbf{I}\right)<2 \eta_{n}\right\} \leq \operatorname{Pr}\left\{\frac{1}{n} \sum_{n_{1} \leq j \leq n_{2}}\left|\log s_{j}\left(\mathbf{F}_{\mathbf{X}}-\alpha \mathbf{I}\right)\right| \geq \frac{1}{2}\right\} \rightarrow 0
$$

by condition (C2). Since a similar estimate holds with $\mathbf{F}_{\mathbf{Y}}$ instead of $\mathbf{F}_{\mathbf{X}}$, it follows that

$$
\operatorname{Pr}\left\{\Omega_{3}^{c}\right\} \leq \operatorname{Pr}\left\{s_{n_{1}}\left(\mathbf{F}_{\mathbf{X}}-\alpha \mathbf{I}\right)<2 \eta_{n}\right\}+\operatorname{Pr}\left\{s_{n_{1}}\left(\mathbf{F}_{\mathbf{Y}}-\alpha \mathbf{I}\right)<2 \eta_{n}\right\} \rightarrow 0 \quad \text { as } n \rightarrow \infty
$$


Furthermore, on the set $\Omega_{3}$ we have, for any $a \in\left[\eta_{n}, 2 \eta_{n}\right]$,

$$
\begin{aligned}
\left|\frac{1}{n} \log \right| & \operatorname{det}\left\{\mathbf{F}_{\mathbf{X}}-\alpha \mathbf{I}\right\}\left|-\frac{1}{n} \log \right| \operatorname{det}\left\{\mathbf{F}_{\mathbf{Y}}-\alpha \mathbf{I}\right\}|| \\
\leq & \frac{1}{n} \sum_{k=n_{2}}^{n}\left|\log s_{k}\left(\mathbf{F}_{\mathbf{X}}-\alpha \mathbf{I}\right)\right|+\frac{1}{n} \sum_{k=n_{2}}^{n}\left|\log s_{k}\left(\mathbf{F}_{\mathbf{Y}}-\alpha \mathbf{I}\right)\right| \\
& +\frac{1}{n} \sum_{k=n_{1}}^{n_{2}-1}\left|\log s_{k}\left(\mathbf{F}_{\mathbf{X}}-\alpha \mathbf{I}\right)\right|+\frac{1}{n} \sum_{k=n_{1}}^{n_{2}-1}\left|\log s_{k}\left(\mathbf{F}_{\mathbf{Y}}-\alpha \mathbf{I}\right)\right| \\
& +\left|\int_{a}^{a^{-1}} \log x d\left(\mathcal{G}_{\mathbf{X}}(x, \alpha)-\mathcal{G}_{\mathbf{Y}}(x, \alpha)\right)\right| \\
& +\int_{\left(2 \eta_{n}\right)^{-1}}^{\infty}|\log x| d\left(\mathcal{G}_{\mathbf{X}}(x, \alpha)+\mathcal{G}_{\mathbf{Y}}(x, \alpha)\right) .
\end{aligned}
$$

Now fix $\varepsilon>0$. The preceding inequality implies that

$$
\begin{aligned}
\operatorname{Pr}\left\{\left|\frac{1}{n} \log \right|\right. & \left.\operatorname{det}\left\{\mathbf{F}_{\mathbf{X}}-\alpha \mathbf{I}\right\}\left|-\frac{1}{n} \log \right| \operatorname{det}\left\{\mathbf{F}_{\mathbf{Y}}-\alpha \mathbf{I}\right\}|| \geq \varepsilon\right\} \\
\leq & \operatorname{Pr}\left\{\Omega_{0}^{c}\right\}+\operatorname{Pr}\left\{\Omega_{1}^{c}\right\}+\operatorname{Pr}\left\{\Omega_{2}^{c}\right\}+\operatorname{Pr}\left\{\Omega_{3}^{c}\right\} \\
& +\operatorname{Pr}\left\{\frac{1}{n} \sum_{k=n_{2}}^{n}\left|\log s_{k}\left(\mathbf{F}_{\mathbf{X}}-\alpha \mathbf{I}\right)\right|+\frac{1}{n} \sum_{k=n_{2}}^{n}\left|\log s_{k}\left(\mathbf{F}_{\mathbf{Y}}-\alpha \mathbf{I}\right)\right| \geq \varepsilon / 4 ; \Omega_{0}\right\} \\
& +\operatorname{Pr}\left\{\frac{1}{n} \sum_{k=n_{1}}^{n_{2}-1}\left|\log s_{k}\left(\mathbf{F}_{\mathbf{X}}-\alpha \mathbf{I}\right)\right|+\frac{1}{n} \sum_{k=n_{1}}^{n_{2}-1}\left|\log s_{k}\left(\mathbf{F}_{\mathbf{Y}}-\alpha \mathbf{I}\right)\right| \geq \varepsilon / 4\right\} \\
& +\operatorname{Pr}\left\{\left|\int_{a}^{a^{-1}} \log x d\left(\mathcal{G}_{\mathbf{X}}(x, \alpha)-\mathcal{G}_{\mathbf{Y}}(x, \alpha)\right)\right| \geq \varepsilon / 4 ; \Omega_{2} \cap \Omega_{1}\right\} \\
& +\operatorname{Pr}\left\{\int_{\left(2 n_{n}\right)^{-1}}^{\infty}|\log x| d\left(\mathcal{G}_{\mathbf{X}}(x, \alpha)+\mathcal{G}_{\mathbf{Y}}(x, \alpha)\right) \geq \varepsilon / 4\right\} .
\end{aligned}
$$

By condition $(C 1)$ and inequalities (4.5) - (4.7),

$$
\operatorname{Pr}\left\{\Omega_{0}^{c}\right\}+\operatorname{Pr}\left\{\Omega_{1}^{c}\right\}+\operatorname{Pr}\left\{\Omega_{2}^{c}\right\}+\operatorname{Pr}\left\{\Omega_{3}^{c}\right\} \rightarrow 0 \quad \text { as } \quad n \rightarrow \infty .
$$

By definition of $\Omega_{0}$ and by condition $(C 2)$, we have, for $n \rightarrow \infty$,

$$
\begin{aligned}
\operatorname{Pr} & \left\{\frac{1}{n} \sum_{k=n_{2}}^{n}\left|\log s_{k}\left(\mathbf{F}_{\mathbf{X}}-\alpha \mathbf{I}\right)\right|+\frac{1}{n} \sum_{k=n_{2}}^{n}\left|\log s_{k}\left(\mathbf{F}_{\mathbf{Y}}-\alpha \mathbf{I}\right)\right| \geq \varepsilon / 4 ; \Omega_{0}\right\} \\
& +\operatorname{Pr}\left\{\frac{1}{n} \sum_{k=n_{1}}^{n_{2}}\left|\log s_{k}\left(\mathbf{F}_{\mathbf{X}}-\alpha \mathbf{I}\right)\right|+\frac{1}{n} \sum_{k=n_{1}}^{n_{2}}\left|\log s_{k}\left(\mathbf{F}_{\mathbf{Y}}-\alpha \mathbf{I}\right)\right| \geq \varepsilon / 4\right\} \rightarrow 0 .
\end{aligned}
$$


Moreover, using that for any $p>0$, the function $x^{-p} \log x$ is decreasing in $x$ for $x \geq \mathrm{e}^{1 / p}$, we get, for large enough $n$,

$$
\begin{aligned}
\int_{\left(2 \eta_{n}\right)^{-1}}^{\infty}|\log x| d\left(\mathcal{G}_{\mathbf{X}}(x, \alpha)+\right. & \left.\mathcal{G}_{\mathbf{Y}}(x, \alpha)\right) \leq C \eta_{n}^{p}\left|\log \eta_{n}\right| \int_{0}^{\infty} x^{p} d\left(\mathcal{G}_{\mathbf{X}}(x, \alpha)+\mathcal{G}_{\mathbf{Y}}(x, \alpha)\right) \\
& \leq C \eta_{n}^{p}\left|\log \eta_{n}\right|\left(\frac{1}{n} \sum_{k=1}^{n} s_{k}^{p}\left(\mathbf{F}_{\mathbf{X}}-\alpha \mathbf{I}\right)+\frac{1}{n} \sum_{k=1}^{n} s_{k}^{p}\left(\mathbf{F}_{\mathbf{Y}}-\alpha \mathbf{I}\right)\right)
\end{aligned}
$$

By the inequality $s_{k}(\mathbf{F}-\alpha \mathbf{I}) \leq s_{k}(\mathbf{F})+|\alpha|$ and condition $(C 0)$, this quantity converges to zero in probability.

Thus, it remains to bound the last but one summand in (4.8). Recall that $a \in\left[\eta_{n}, 2 \eta_{n}\right]$. Integrating by parts, we have

$$
\begin{aligned}
\left|\int_{a}^{a^{-1}} \log x d\left(\mathcal{G}_{\mathbf{X}}(x, \alpha)-\mathcal{G}_{\mathbf{Y}}(x, \alpha)\right)\right| \leq & |\log a|\left|\mathcal{G}_{\mathbf{X}}(a, \alpha)-\mathcal{G}_{\mathbf{Y}}(a, \alpha)\right| \\
& +|\log a|\left|\mathcal{G}_{\mathbf{X}}\left(a^{-1}, \alpha\right)-\mathcal{G}_{\mathbf{Y}}\left(a^{-1}, \alpha\right)\right| \\
& +\left|\int_{a}^{a^{-1}} \frac{\mathcal{G}_{\mathbf{X}}(x, \alpha)-\mathcal{G}_{\mathbf{Y}}(x, \alpha)}{x} d x\right|
\end{aligned}
$$

Recall that we need to bound this expression for $\omega \in \Omega_{2} \cap \Omega_{1}$ only and that $\varkappa_{n} \leq \eta_{n}$ for such $\omega$. By Chebyshev's inequality, we have

$$
\max \left\{1-\mathcal{G}_{\mathbf{X}}(M, \alpha), 1-\mathcal{G}_{\mathbf{Y}}(M, \alpha)\right\} \leq \frac{1}{M^{p}}\left(\frac{1}{n} \sum_{k=1}^{n} s_{k}^{p}\left(\mathbf{F}_{\mathbf{X}}-\alpha \mathbf{I}\right)+\frac{1}{n} \sum_{k=1}^{n} s_{k}^{p}\left(\mathbf{F}_{\mathbf{Y}}-\alpha \mathbf{I}\right)\right)
$$

for any $M>0$. It therefore follows from condition $(C 0)$ that the second term on the r.h.s. of (4.11) converges to zero in probability. Furthermore, by the definition of $\varkappa_{n}$, we have the following bound

$$
\left|\mathcal{G}_{\mathbf{X}}(x, \alpha)-\mathcal{G}_{\mathbf{Y}}(x, \alpha)\right| \leq \varkappa_{n}+\mathcal{G}_{\mathbf{Y}}\left(x+\varkappa_{n}, \alpha\right)-\mathcal{G}_{\mathbf{Y}}\left(x-\varkappa_{n}, \alpha\right), \quad x \in \mathbb{R} .
$$

Thus, for $\omega \in \Omega_{2} \cap \Omega_{1}$ we may find $a \in\left[\eta_{n}, 2 \eta_{n}\right]$ such that

$$
\left|\mathcal{G}_{\mathbf{X}}(a, \alpha)-\mathcal{G}_{\mathbf{Y}}(a, \alpha)\right| \leq \varkappa_{n}+\mathcal{G}_{\mathbf{Y}}\left(a+\varkappa_{n}, \alpha\right)-\mathcal{G}_{\mathbf{Y}}\left(a-\varkappa_{n}, \alpha\right) \leq \varkappa_{n}+\varkappa_{n}^{\frac{1}{2}} \leq 2 \varkappa_{n}^{\frac{1}{2}} \leq 2 \eta_{n}^{\frac{1}{2}} .
$$

Because $\eta_{n}^{\frac{1}{2}}\left|\log \eta_{n}\right| \rightarrow 0$ as $n \rightarrow \infty$, it follows that the first term on the r.h.s of (4.11) converges to zero in probability. Finally, using inequality (4.12) again, we obtain

$$
\begin{aligned}
& \mid \int_{a}^{a^{-1}} \frac{\mathcal{G}_{\mathbf{X}}(x, \alpha)-\mathcal{G}_{\mathbf{Y}}(x, \alpha)}{x} d x \mid \leq \int_{\eta_{n}}^{\eta_{n}^{-1}} \frac{\left|\mathcal{G}_{\mathbf{X}}(x, \alpha)-\mathcal{G}_{\mathbf{Y}}(x, \alpha)\right|}{x} d x \\
& \leq C \varkappa_{n}\left|\log \eta_{n}\right|+\int_{\eta_{n}}^{\eta_{n}^{-1}} \frac{\mathcal{G}_{\mathbf{Y}}\left(x+\varkappa_{n}, \alpha\right)-\mathcal{G}_{\mathbf{Y}}\left(x-\varkappa_{n}, \alpha\right)}{x} d x .
\end{aligned}
$$


It is straightforward to check that for any $0<\varepsilon<a<b$ and any distribution function $F(x)$ the following inequality

$$
\int_{a}^{b} \frac{F(x+\varepsilon)-F(x-\varepsilon)}{x} d x \leq 2 \varepsilon\left(\frac{1}{a-\varepsilon}+\frac{1}{b-\varepsilon}\right) \leq \frac{4 \varepsilon}{a-\varepsilon}
$$

holds. Applying this inequality, we get

$$
\int_{\eta_{n}}^{\eta_{n}^{-1}} \frac{\mathcal{G}_{\mathbf{Y}}\left(x+\varkappa_{n}, \alpha\right)-\mathcal{G}_{\mathbf{Y}}\left(x-\varkappa_{n}, \alpha\right)}{x} d x \leq \frac{C \varkappa_{n}}{\eta_{n}-\varkappa_{n}} .
$$

Therefore, for $\omega \in \Omega_{2} \cap \Omega_{1}$ we obtain, for large enough $n$,

$$
\left|\int_{a}^{a^{-1}} \frac{\mathcal{G}_{\mathbf{X}}(x, \alpha)-\mathcal{G}_{\mathbf{Y}}(x, \alpha)}{x} d x\right| \leq C\left(\varkappa_{n}\left|\log \eta_{n}\right|+\frac{\varkappa_{n}}{\eta_{n}-\varkappa_{n}}\right) \leq C \eta_{n}^{1 / 2} .
$$

This implies that

$$
\lim _{n \rightarrow \infty} \operatorname{Pr}\left\{\left|\int_{a}^{a^{-1}} \log x d\left(\mathcal{G}_{\mathbf{X}}(x, \alpha)-\mathcal{G}_{\mathbf{Y}}(x, \alpha)\right)\right| \geq \varepsilon / 4 ; \Omega_{2} \cap \Omega_{1}\right\}=0 .
$$

Thus relation (4.3) is proved.

We may now apply the "replacement principle" by Tao and Vu; see 41, Theorem 2.1. Note that this theorem is based on two assumptions (i) and (ii). Assumption (ii) is just a reformulation of relation (4.3). Assumption (i) is only needed to show that the probability measures $\mu_{\mathbf{X}}$ and $\mu_{\mathbf{Y}}$ are tight in probability (see Equations (3.3) and (3.4) in [41]), and may be replaced with our assumption $(C 0)$. It therefore follows that $\mu_{\mathbf{X}}-\mu_{\mathbf{Y}}$ converges weakly to zero in probability, i.e. for any bounded continuous function $f$ and any $\varepsilon>0$, we have

$$
\lim _{n \rightarrow \infty} \operatorname{Pr}\left\{\left|\int_{\mathbb{C}} f d \mu_{\mathbf{X}}-\int_{\mathbb{C}} f d \mu_{\mathbf{Y}}\right|>\varepsilon\right\}=0
$$

Thus Theorem 4.4 is proved.

Remark 4.5. It follows from the proof of Theorem 4.4 that it suffices to assume, instead of the conditions (3.1), (3.2), and (3.20) - (3.25) of Theorem 3.2, that for any $\alpha \in \mathbb{C}$,

$$
\lim _{n \rightarrow \infty} L\left(\mathcal{G}_{\mathbf{X}}(\cdot, \alpha), \mathcal{G}_{\mathbf{Y}}(\cdot, \alpha)\right)=0 \quad \text { in probability. }
$$

\section{Asymptotic Freeness of Random Matrices}

In this section we consider the asymptotic freeness of random matrices with special structure. Before that, we recall the definition of Voiculescu's asymptotic freeness as well as some basic notions from free probability theory. See also the survey by Speicher [40] and the lecture notes by Voiculescu [46]. 
Definition 5.1. A pair $(\mathcal{A}, \varphi)$ consisting of a unital algebra $\mathcal{A}$ and a linear functional $\varphi: \mathcal{A} \rightarrow \mathbb{C}$ with $\varphi(1)=1$ is called a non-commutative probability space. Elements of $\mathcal{A}$ are called random variables, the numbers $\varphi\left(a_{i(1)} \cdots a_{i(n)}\right)$ for such random variables $a_{1}, \ldots, a_{k} \in \mathcal{A}$ are called moments, and the collection of all moments is called the joint distribution of $a_{1}, \ldots, a_{k}$. Equivalently, we may say that the joint distribution of $a_{1}, \ldots, a_{k}$ is given by the linear functional $\mu_{a_{1}, \ldots, a_{k}}: \mathbb{C}\left\langle X_{1}, \ldots, X_{k}\right\rangle \rightarrow \mathbb{C}$ with $\mu_{a_{1}, \ldots, a_{k}}\left(P\left(X_{1}, \ldots, X_{k}\right)\right)=\varphi\left(P\left(a_{1}, \ldots, a_{k}\right)\right)$, where $\mathbb{C}\left\langle X_{1}, \ldots, X_{k}\right\rangle$ denotes the algebra of all polynomials in $k$ non-commuting indeterminates $X_{1}, \ldots, X_{k}$.

If, for a given element $a \in \mathcal{A}$, there exists a unique probability measure $\mu_{a}$ on $\mathbb{R}$ such that $\int t^{k} d \mu_{a}(t)=\varphi\left(a^{k}\right)$ for all $k \in \mathbb{N}$, we identify the distribution of $a$ with the probability measure $\mu_{a}$.

Definition 5.2. Let $(\mathcal{A}, \varphi)$ be a non-commutative probability space.

1) Let $\left(\mathcal{A}_{i}\right)_{i \in I}$ be a family of unital sub-algebras of $\mathcal{A}$. The sub-algebras $\mathcal{A}_{i}$ are called free or freely independent, if, for any positive integer $k, \varphi\left(a_{1} \cdots a_{k}\right)=0$ whenever the following set of conditions holds: $a_{j} \in \mathcal{A}_{i(j)}$ (with $i(j) \in I$ ) for all $j=1, \ldots, k, \varphi\left(a_{j}\right)=0$ for all $j=1, \ldots, k$, and neighbouring elements are from taken different sub-algebras, i.e. $i(1) \neq i(2), i(2) \neq i(3), \ldots, i(k-1) \neq i(k)$.

2) Let $\left(\mathcal{A}_{i}^{\prime}\right)_{i \in I}$ be a family of subsets of $\mathcal{A}$. The subsets $\mathcal{A}_{i}^{\prime}$ are called free or freely independent, if their generated unital sub-algebras are free, i.e. if $\left(\mathcal{A}_{i}\right)_{i \in I}$ are free, where, for each $i \in I, \mathcal{A}_{i}$ is the smallest unital sub-algebra of $\mathcal{A}$ which contains $\mathcal{A}_{i}^{\prime}$.

3) Let $\left(a_{i}\right)_{i \in I}$ be a family of elements from $\mathcal{A}$. The elements $a_{i}$ are called free or freely independent, if the subsets $\left\{a_{i}\right\}$ are free.

Consider two random variables $a$ and $b$ which are free. Then the distributions of $a+b$ and $a b$ (in the sense of linear functionals) depend only on the distributions of $a$ and $b$ (see e.g. [19], Chapter 2), and we can make the following definition:

Definition 5.3. For free random variables $a$ and $b$, the distributions of $a+b$ and $a b$ are called the free additive convolution and the free multiplicative convolution of $\mu_{a}$ and $\mu_{b}$ and are denoted by

$$
\mu_{a+b}=\mu_{a} \boxplus \mu_{b} \quad \text { and } \quad \mu_{a b}=\mu_{a} \otimes \mu_{b}
$$

respectively.

It can be shown (see e.g. [19], Chapter 3) that this defines commutative and associative operations. Furthermore, it is well known that if $\mu_{a}$ and $\mu_{b}$ are compactly supported probability measures on $\mathbb{R}$, then $\mu_{a} \boxplus \mu_{b}$ is also a compactly supported probability measure on $\mathbb{R}$. Similarly, if $\mu_{a}$ and $\mu_{b}$ are compactly supported probability measure on $\mathbb{R}_{+}$, then $\mu_{a} \otimes \mu_{b}$ is also a compactly supported probability measure on $\mathbb{R}_{+}$.

We shall now define the $R$-transform and the $S$-transform of a random variable $a \in \mathcal{A}$ as introduced by Voiculescu (see e.g. [46], p. 296 and p. 310). Let $a \in \mathcal{A}$ be a random variable with distribution $\mu_{a}$ on $\mathbb{R}$. Let $M_{a}(z)$ denote the generic formal moment generating function of $\mu_{a}$, namely $M_{a}(z)=\sum_{k=1}^{\infty} m_{k} z^{k}$, where $m_{k}=\int_{-\infty}^{\infty} x^{k} d \mu_{a}(x)=$ 
$\varphi\left(a^{k}\right)$. Let $G_{a}(z):=\frac{1}{z}\left(1+M_{a}\left(\frac{1}{z}\right)\right)$, and define the $R$-transform of $a$ by

$$
R_{a}(z):=G_{a}^{-1}(z)-\frac{1}{z}
$$

where $G_{a}^{-1}(z)$ denotes the inverse of $G_{a}(z)$ w.r.t. composition of functions. Moreover, when $\varphi(a) \neq 0$, define the $S$-transform of $a$ by

$$
S_{a}(z):=\frac{z+1}{z} M_{a}^{-1}(z),
$$

where $M_{a}^{-1}(z)$ denotes the inverse of $M_{a}(z)$ w.r.t. composition of functions. These definitions have to be understood at the level of formal series. However, when $a$ has a distribution on $\mathbb{R}$ with compact support, $R_{a}(z)$ and $S_{a}(z)$ may be regarded as analytic functions in a certain neighborhood of the origin.

Then, for free random variables $a$ and $b$, we have

$$
R_{a+b}(z)=R_{a}(z)+R_{b}(z)
$$

and, when $\phi(a) \neq 0$ and $\phi(b) \neq 0$,

$$
S_{a b}(z)=S_{a}(z) S_{b}(z)
$$

see e.g. [46], p. 296 and p. 310.

For a generalization of the $S$-transform to the case where $\varphi(a)=0$ and $\varphi\left(a^{2}\right) \neq 0$, see Rao and Speicher [37] as well as Arizmendi and Pérez-Abreu [6]. Here the $S$-transform is a formal power series in $\sqrt{z}$, and there exist two branches of the $S$-transform. We will use this generalization only in the case where $a$ has a symmetric distribution $\mu_{a} \neq \delta_{0}$ on $\mathbb{R}$, in which we adopt the approach by Arizmendi and Pérez-Abreu [6]; see definition (5.13) below.

Let $a$ have a compactly supported distribution $\mu_{a} \neq \delta_{0}$ on $\mathbb{R}$, and define the function $\widetilde{R}_{a}(z):=z R_{a}(z)$. Then

$$
z S_{a}(z)=\widetilde{R}_{a}^{-1}(z)
$$

where $\widetilde{R}_{a}^{-1}(z)$ denotes the inverse of $\widetilde{R}_{a}(z)$ w.r.t. composition of functions. See for instance Nica [34], Equation (21) in Chapter 13. For clarity, let us emphasize that we call $\widetilde{R}_{a}(z)$ what is called $R_{a}(z)$ in [34]. Furthermore, let us note that the argument in [34] requires that $\varphi(a) \neq 0$. However, when $\varphi(a)=0$ and $\varphi\left(a^{2}\right) \neq 0$, one can use similar arguments as in Rao and Speicher [37] to show that, similarly as for the $S$-transform, there exist two branches of $\widetilde{R}_{a}^{-1}(z)$ and that (5.5) continues to hold with an appropriate choice of these branches. We shall always take the branches such that

$$
\operatorname{Im} \widetilde{R}_{a}^{-1}(z) \leq 0 \text { and } \operatorname{Im} S_{a}(z) \geq 0 \text { for } z \approx 0, z \notin \mathbb{R}_{+} .
$$


For example, when the distribution of $a$ is the semi-circular law, we take $\widetilde{R}_{a}^{-1}(z)=-\sqrt{z}$ and $S_{a}(z)=-\frac{1}{\sqrt{z}}$, where the branch of $\sqrt{z}$ is chosen such that $\operatorname{Im} \sqrt{z} \geq 0$ for $z \in \mathbb{C} \backslash \mathbb{R}_{+}$. Remark 5.4. The $R$-transform and the $S$-transform (as well as the other transforms) can also be introduced for a compactly supported probability measure $\mu$ on $\mathbb{R}$ and will then be denoted by $R_{\mu}$ and $S_{\mu}$, respectively. For the $S$-transform, we require that $\mu \neq \delta_{0}$.

We will also need the additive and multiplicative convolution for probability measures with unbounded support; see Bercovici and Voiculescu [11 for details. For our purposes, the following definitions are convenient: For any probability measures $\mu, \nu$ on $\mathbb{R}$, we set

$$
\mu \boxplus \nu:=\lim _{n \rightarrow \infty}\left(\mu_{n} \boxplus \nu_{n}\right),
$$

where $\left(\mu_{n}\right),\left(\nu_{n}\right)$ are sequences of probability measures on $\mathbb{R}$ with bounded support which converge to $\mu, \nu$ w.r.t. Lévy distance $d_{L}$. Similarly, for any probability measures $\mu, \nu$ on $\mathbb{R}_{+}$, we set

$$
\mu \otimes \nu:=\lim _{n \rightarrow \infty}\left(\mu_{n} \otimes \nu_{n}\right)
$$

where $\left(\mu_{n}\right),\left(\nu_{n}\right)$ are sequences of probability measures on $\mathbb{R}_{+}$with bounded support which converge to $\mu, \nu$ w.r.t. Kolmogorov distance $d_{K}$. It can be shown that these operations are well-defined and continuous in the sense that

$$
d_{L}\left(\mu_{1} \boxplus \nu_{1}, \mu_{2} \boxplus \nu_{2}\right) \leq d_{L}\left(\mu_{1}, \mu_{2}\right)+d_{L}\left(\nu_{1}, \nu_{2}\right)
$$

and

$$
d_{K}\left(\mu_{1} \otimes \nu_{1}, \mu_{2} \otimes \nu_{2}\right) \leq d_{K}\left(\mu_{1}, \mu_{2}\right)+d_{K}\left(\nu_{1}, \nu_{2}\right),
$$

cf. Propositions 4.13 and 4.14 in [11.

The above transforms also have extensions to unbounded probability measures. Let us provide the details for the $S$-transform; cf. Section 6 in [11]. For a probability measure $\nu$ on $(0, \infty)$, define the function

$$
\psi_{\nu}(z):=\int_{0}^{\infty} \frac{t z}{1-t z} d \nu(t)
$$

By Proposition 6.2 in [11, this function is univalent in the left half-plane $i \mathbb{C}^{+}$, with $\psi_{\nu}\left(i \mathbb{C}^{+}\right) \cap \mathbb{R}=(-1,0)$. The $S$-transform of $\nu$ is the function on $\psi_{\nu}\left(i \mathbb{C}^{+}\right)$defined by

$$
S_{\nu}=\frac{z+1}{z} \psi_{\nu}^{-1}(z)
$$

where $\psi_{\nu}^{-1}$ denotes the inverse of $\psi_{\nu}$. 
Finally, we also need the $S$-transform for symmetric probability measures on $\mathbb{R} \backslash\{0\}$. Here we follow Arizmendi and Pérez-Abreu [6]. Note that the function

$$
\begin{aligned}
& \mathcal{Q}:\{\text { symmetric p.m.'s on } \mathbb{R} \backslash\{0\}\} \rightarrow \quad\{\text { p.m.'s on }(0, \infty)\} \\
& \mu \quad \mapsto \quad \text { induced measure of } \mu \\
& \text { under the mapping } x \mapsto x^{2}
\end{aligned}
$$

is one-to-one. For a symmetric probability measure $\mu$ on $\mathbb{R} \backslash\{0\}$, we define the $S$-transform by

$$
S_{\mu}(z)=\sqrt{\frac{z+1}{z} S_{\mathcal{Q}(\mu)}(z)},
$$

where the branch of the square-root is such that $\operatorname{Im} S_{\mu}(z) \geq 0$ for $z \in(-1,0)$. Of course, for probability measures with compact support (and the respective additional properties), the new definitions (5.11) and (5.13) are consistent with the previous ones. Also, let us mention that the assumption $\mu(0)=0$ is more restrictive than necessary (it could be relaxed to $\mu(0)<1)$, but sufficient for our purposes.

Let $\mu, \mu_{1}, \mu_{2}, \mu_{3}, \ldots$ be a family either of probability measures on $(0, \infty)$ or of symmetric probability measures on $\mathbb{R} \backslash\{0\}$. Then we have the following results:

$$
\begin{aligned}
& \text { The } S \text {-transform } S_{\mu} \text { determines the measure } \mu \text {. } \\
& \text { If } \mu_{n} \Rightarrow \mu \text { then } S_{\mu_{n}} \rightarrow S_{\mu} \text { locally uniformly on }(-1,0) \text {. }
\end{aligned}
$$

Next, we recall Voiculescu's definition of asymptotic freeness for random matrices. Note that for any (classical) probability space $(\Omega, \mathcal{F}, \operatorname{Pr})$, the set $\mathcal{A}:=\mathcal{M}_{n \times n}\left(L^{\infty-}(\Omega)\right)$ of $n \times n$ matrices with entries in $L^{\infty-}(\Omega)$ endowed with the functional $\varphi(\mathbf{A}):=\mathbf{E} \frac{1}{n} \operatorname{Tr}(\mathbf{A})$ is a non-commutative probability space.

Definition 5.5. Let $I$ be an index set, and let $I=I_{1} \cup \cdots \cup I_{l}$ be a partition of $I$. For each $n \in \mathbb{N}$, let $\left(\mathbf{A}_{n, i}\right)_{i \in I}$ a family of random matrices in $\mathcal{M}_{n \times n}\left(L^{\infty-}(\Omega)\right)$. The families $\left\{\mathbf{A}_{n, i}: i \in I_{j}\right\}, j=1, \ldots, l$, are called asymptotically free if there exists a family $\left(a_{i}\right)_{i \in I}$ of non-commutative random variables in some non-commutative probability space $(\mathcal{A}, \varphi)$ such that for all $k \in \mathbb{N}$ and all $i(1), \ldots, i(k) \in I$,

$$
\lim _{n \rightarrow \infty} \frac{1}{n} \mathbf{E} \operatorname{Tr}\left(\mathbf{A}_{n, i(1)} \cdots \mathbf{A}_{n, i(k)}\right)=\varphi\left(a_{i(1)} \cdots a_{i(k)}\right)
$$

and the families $\left\{a_{i}: i \in I_{j}\right\}, j=1, \ldots, l$, are free.

Remark 5.6. Let $\left(\mathbf{A}_{n}\right)_{n \in \mathbb{N}}$ and $\left(\mathbf{B}_{n}\right)_{n \in \mathbb{N}}$ be sequences of self-adjoint random matrices with $\mathbf{A}_{n}, \mathbf{B}_{n} \in \mathcal{M}_{n \times n}\left(L^{\infty-}(\Omega)\right)$ such that the mean eigenvalue distributions of $\mathbf{A}_{n}$ and $\mathbf{B}_{n}$ converge in moments to compactly supported p.m.'s $\mu_{\mathbf{A}}$ and $\mu_{\mathbf{B}}$, respectively, and $\left(\mathbf{A}_{n}\right)_{n \in \mathbb{N}}$ and $\left(\mathbf{B}_{n}\right)_{n \in \mathbb{N}}$ are asymptotically free. Then the mean eigenvalue distribution of $\mathbf{A}_{n}+\mathbf{B}_{n}$ converges in moments to the p.m. $\mu_{\mathbf{A}} \boxplus \mu_{\mathbf{B}}$. Moreover, if $\mathbf{A}_{n}$ and $\mathbf{B}_{n}$ are additionally positive semi-definite, the mean eigenvalue distribution of $\mathbf{A}_{n} \mathbf{B}_{n}$ converges in moments to the p.m. $\mu_{\mathbf{A}} \otimes \mu_{\mathbf{B}}$. 
Remark 5.7. It is easy to see that two sequences $\left(\mathbf{A}_{n}\right)_{n \in \mathbb{N}}$ and $\left(\mathbf{B}_{n}\right)_{n \in \mathbb{N}}$ with $\mathbf{A}_{n}, \mathbf{B}_{n}$ $\in \mathcal{M}_{n \times n}\left(L^{\infty-}(\Omega)\right)$ are asymptotically free if and only if for all $k \geq 1$ and all $j_{1}, l_{1}, \ldots, j_{k}, l_{k}$ $\geq 1$, the following relation holds, assuming that all limits involved exist,

$$
\begin{aligned}
& \lim _{n \rightarrow \infty} \mathbf{E} \frac{1}{n} \operatorname{Tr}(\left(\mathbf{A}_{n}^{j_{1}}-\left(\lim _{\nu \rightarrow \infty} \frac{1}{\nu} \mathbf{E} \operatorname{Tr} \mathbf{A}_{\nu}^{j_{1}}\right) \mathbf{I}_{n}\right)\left(\mathbf{B}_{n}^{l_{1}}-\left(\lim _{\nu \rightarrow \infty} \frac{1}{\nu} \mathbf{E} \operatorname{Tr} \mathbf{B}_{\nu}^{l_{1}}\right) \mathbf{I}_{n}\right) \cdots \\
&\left.\left(\mathbf{A}_{n}^{j_{k}}-\left(\lim _{\nu \rightarrow \infty} \frac{1}{\nu} \mathbf{E} \operatorname{Tr} \mathbf{A}_{\nu}^{j_{k}}\right) \mathbf{I}_{n}\right)\left(\mathbf{B}_{n}^{l_{k}}-\left(\lim _{\nu \rightarrow \infty} \frac{1}{\nu} \mathbf{E} \operatorname{Tr} \mathbf{B}_{\nu}^{l_{k}}\right) \mathbf{I}_{n}\right)\right)=0 .
\end{aligned}
$$

Here $\mathbf{I}_{n}$ denotes the identity matrix of dimension $n \times n$.

Consider a sequence of random matrices $\left(\mathbf{Y}_{n}\right)_{n \in \mathbb{N}}$ with $\mathbf{Y}_{n} \in \mathcal{M}_{n \times n}\left(L^{\infty-}(\Omega)\right), n \in \mathbb{N}$. Suppose that the matrix $\mathbf{Y}_{n}$ is bi-unitary invariant, i.e. the joint distribution of the entries of $\mathbf{Y}_{n}$ is equal to that of the entries of $\mathbf{V}_{1} \mathbf{Y}_{n} \mathbf{V}_{2}$, for any unitary $n \times n$ matrices $\mathbf{V}_{1}$ and $\mathbf{V}_{2}$. (For instance, this is the case if $\mathbf{Y}_{n}$ is a (non-self-adjoint) matrix with independent standard complex Gaussian entries.) Introduce the matrices

$$
\mathbf{A}_{n}=\left[\begin{array}{cc}
\mathbf{O} & \mathbf{Y}_{n} \\
\mathbf{Y}_{n}^{*} & \mathbf{O}
\end{array}\right]
$$

and, for $\alpha=u+i v$ with real $u, v$,

$$
\mathbf{B}_{n}=\mathbf{J}_{n}(\alpha)=\left[\begin{array}{cc}
\mathbf{O} & -\alpha \mathbf{I}_{n} \\
-\bar{\alpha} \mathbf{I}_{n} & \mathbf{O}
\end{array}\right]
$$

We shall investigate the asymptotic freeness of the matrices $\left(\mathbf{A}_{n}\right)_{n \in \mathbb{N}}$ and $\left(\mathbf{B}_{n}\right)_{n \in \mathbb{N}}$.

Proposition 5.8. Let $\left(\mathbf{A}_{n}\right)_{n \in \mathbb{N}}$ and $\left(\mathbf{B}_{n}\right)_{n \in \mathbb{N}}$ be sequences of matrices as above, where for each $n \in \mathbb{N}, \mathbf{Y}_{n}$ is a bi-unitary invariant matrix, and $\alpha$ is a fixed complex number. Moreover, suppose that the eigenvalue distribution of $\mathbf{A}_{n}$ converges weakly in probability (as $n \rightarrow \infty$ ) to a compactly supported probability measure $\mu$ on $\mathbb{R}$ and that for any $k \in \mathbb{N}$, $\sup _{n \in \mathbb{N}} \frac{1}{n} \mathbf{E} \operatorname{Tr} A_{n}^{2 k}<\infty$. Then the sequences $\left(\mathbf{A}_{n}\right)_{n \in \mathbb{N}}$ and $\left(\mathbf{B}_{n}\right)_{n \in \mathbb{N}}$ are asymptotically free.

Proof. We check Equation (5.16). Note that

$$
\mathbf{B}_{n}^{l}=\mathbf{J}_{n}^{l}(\alpha)= \begin{cases}|\alpha|^{2 p} \mathbf{I}_{2 n}, & \text { if } l=2 p, \\ |\alpha|^{2 p} \mathbf{J}_{n}(\alpha), & \text { if } l=2 p+1 .\end{cases}
$$

From here it follows immediately that

$$
\mathbf{J}_{n}^{2 p}(\alpha)-\left(\lim _{\nu \rightarrow \infty} \frac{1}{2 \nu} \mathbf{E} \operatorname{Tr} \mathbf{J}_{\nu}^{2 p}(\alpha)\right) \mathbf{I}_{2 n}=\mathbf{O} .
$$

This means that relation (5.16) holds if at least one of the $l_{1}, l_{2}, \ldots, l_{k}$ is even. Hence, suppose that $l_{1}, \ldots, l_{k}$ are all odd. In this case we may reduce relation (5.16) to

$$
\lim _{n \rightarrow \infty} \mathbf{E} \frac{1}{n} \operatorname{Tr}\left(\widetilde{\mathbf{A}_{n}^{j_{1}}} \mathbf{J}(\alpha) \cdots \widetilde{\mathbf{A}_{n}^{j_{k}}} \mathbf{J}(\alpha)\right)=0,
$$

where

$$
\widetilde{\mathbf{A}_{n}^{j}}=\mathbf{A}_{n}^{j}-\left(\lim _{\nu \rightarrow \infty} \frac{1}{2 \nu} \mathbf{E} \operatorname{Tr} \mathbf{A}_{\nu}^{j}\right) \mathbf{I}_{2 n} .
$$


Note that

$$
\mathbf{A}_{n}^{j}= \begin{cases}{\left[\begin{array}{cc}
\left(\mathbf{Y}_{n} \mathbf{Y}_{n}^{*}\right)^{p} & \mathbf{O} \\
\mathbf{O} & \left(\mathbf{Y}_{n}^{*} \mathbf{Y}_{n}\right)^{p}
\end{array}\right],} & \text { if } j=2 p \\
{\left[\begin{array}{cc}
\mathbf{O} & \left(\mathbf{Y}_{n} \mathbf{Y}_{n}^{*}\right)^{p} \mathbf{Y}_{n} \\
\left(\mathbf{Y}_{n}^{*} \mathbf{Y}_{n}\right)^{p} \mathbf{Y}_{n}^{*} & \mathbf{O}
\end{array}\right],} & \text { if } j=2 p+1 .\end{cases}
$$

In order to complete the proof, we proceed similarly as in Hiai and Petz [27]. Using the bi-unitary invariance and the singular value decomposition of the matrix $\mathbf{Y}_{n}$, we may represent the matrix $\mathbf{Y}_{n}$ as $\mathbf{U}_{n} \boldsymbol{\Delta}_{n} \mathbf{V}_{n}^{*}$, where $\mathbf{U}_{n}, \boldsymbol{\Delta}_{n}, \mathbf{V}_{n}$ are independent, $\mathbf{U}_{n}$ and $\mathbf{V}_{n}$ are random unitary matrices (with Haar distribution), and $\boldsymbol{\Delta}_{n}$ is a random diagonal matrix whose diagonal elements are the singular values of $\mathbf{Y}_{n}$, but with random signs (chosen uniformly at random and independently from everything else). Note that

$$
\widetilde{\mathbf{A}_{n}^{j}}=\left\{\begin{array}{cc}
{\left[\begin{array}{cc}
\mathbf{U}_{n}\left(\boldsymbol{\Delta}_{n}^{2 p}-\int x^{2 p} d \mu \mathbf{I}\right) \mathbf{U}_{n}^{*} & \mathbf{O} \\
\mathbf{O} & \mathbf{V}_{n}\left(\boldsymbol{\Delta}_{n}^{2 p}-\int x^{2 p} d \mu \mathbf{I}\right) \mathbf{V}_{n}^{*}
\end{array}\right],} & \text { if } j=2 p, \\
{\left[\begin{array}{cc}
\mathbf{O} & \mathbf{U}_{n} \boldsymbol{\Delta}_{n}^{2 p+1} \mathbf{V}_{n}^{*} \\
\mathbf{V}_{n} \boldsymbol{\Delta}_{n}^{2 p+1} \mathbf{U}_{n}^{*} & \mathbf{O}
\end{array}\right],} & \text { if } j=2 p+1 .
\end{array}\right.
$$

Thus, the non-zero $n \times n$ blocks in the matrix $\widetilde{\mathbf{A}_{n}^{j_{1}}} \mathbf{J}(\alpha) \cdots \widetilde{\mathbf{A}_{n}^{j_{k}}} \mathbf{J}(\alpha)$ in $(15.18)$ are products of the matrices $\mathbf{U}_{n}\left(\boldsymbol{\Delta}_{n}^{2 p}-\int x^{2 p} d \mu \mathbf{I}\right) \mathbf{U}_{n}^{*}, \mathbf{V}_{n}\left(\boldsymbol{\Delta}_{n}^{2 p}-\int x^{2 p} d \mu \mathbf{I}\right) \mathbf{V}_{n}^{*}, \mathbf{U}_{n} \boldsymbol{\Delta}_{n}^{2 p+1} \mathbf{V}_{n}^{*}, \mathbf{V}_{n} \boldsymbol{\Delta}_{n}^{2 p+1} \mathbf{U}_{n}^{*}$, as well as certain powers of $\alpha$ and $\bar{\alpha}$, such that each $\mathbf{U}_{n}^{*}$ is followed by a $\mathbf{V}_{n}$, and each $\mathbf{V}_{n}^{*}$ is followed by a $\mathbf{U}_{n}$.

Now, by our assumptions, it is easy to see that the eigenvalue distribution of $\boldsymbol{\Delta}_{n}$ converges weakly in probability to $\mu$. Furthermore, since the matrices $\mathbf{A}_{n}^{2}$ and $\boldsymbol{\Delta}_{n}^{2}$ have the same eigenvalue distributions, we have $\sup _{n \in \mathbb{N}} \frac{1}{n} \mathbf{E} \operatorname{Tr} \boldsymbol{\Delta}_{n}^{2 k}<\infty$ for each $k \in \mathbb{N}$. Starting from these observations, it is straightforward to show that

$$
\lim _{n \rightarrow \infty} \frac{1}{n} \operatorname{Tr} \Delta_{n}^{k}=\int x^{k} d \mu(x) \quad \text { in probability }
$$

for each $k \in \mathbb{N}$. Therefore, by a diagonalization argument, we may find a subsequence $\left(\boldsymbol{\Delta}_{n_{l}}\right)$ such that

$$
\lim _{l \rightarrow \infty} \frac{1}{n_{l}} \operatorname{Tr} \boldsymbol{\Delta}_{n_{l}}^{k}=\int x^{k} d \mu(x) \quad \text { almost surely }
$$

for each $k \in \mathbb{N}$. Now, applying Theorem 2.1 in [27] conditionally on the sequence $\left(\boldsymbol{\Delta}_{n}\right)$, we obtain that the families $\left\{\mathbf{U}_{n_{l}}, \mathbf{U}_{n_{l}}^{*}\right\},\left\{\mathbf{V}_{n_{l}}, \mathbf{V}_{n_{l}}^{*}\right\}$ and $\left\{\boldsymbol{\Delta}_{n_{l}}\right\}$ are asymptotically free almost surely. (Strictly speaking, we may not apply Theorem 2.1 directly, but we first have to replace the matrices $\boldsymbol{\Delta}_{n_{l}}$ with matrices of uniformly bounded operator norm, as in the proofs of Theorems 3.2 and 4.3 in [27].) Since we may repeat the preceding 
argument for any subsequence of the original sequence $\left(\boldsymbol{\Delta}_{n}\right)$, we come to the conclusion that the families $\left\{\mathbf{U}_{n}, \mathbf{U}_{n}^{*}\right\},\left\{\mathbf{V}_{n}, \mathbf{V}_{n}^{*}\right\}$ and $\left\{\boldsymbol{\Delta}_{n}\right\}$ are also asymptotically free almost surely. Finally, as $\sup _{n \in \mathbb{N}} \frac{1}{n} \mathbf{E} \operatorname{Tr} \boldsymbol{\Delta}_{n}^{2 k}<\infty$ for each $k \in \mathbb{N}$, these families are asymptotically free in the ordinary sense as well.

But this implies that the limit in (5.18) is equal to zero. This completes the proof of the asymptotic freeness of $\mathbf{A}_{n}$ and $\mathbf{B}_{n}$.

Remark 5.9. In our applications of Proposition 5.8, the matrices $\mathbf{Y}_{n}$ will be products of Gaussian matrices with independent entries and / or matrices of uniformly bounded operator norm. It is easy to see that in this case the assumption that $\sup \frac{1}{n} \mathbf{E} \operatorname{Tr} \mathbf{A}_{n}^{2 k}<\infty$ for each $k \in \mathbb{N}$ is satisfied.

Remark 5.10. The notion of bi-unitary invariance is relevant for computing the limiting spectral distributions for random matrices with i.i.d. standard complex Gaussian entries. To compute the limiting distributions for random matrices with i.i.d. standard real Gaussian entries we need the notion of bi-orthogonal invariance. According to a side-remark in Hiai and Petz [27], the results for this case are analogous.

Moreover, using bi-orthogonal invariance, it is even possible to treat random matrices with i.i.d. entries with a common bivariate real Gaussian distribution. (Thus, we may allow for correlations between the real and imaginary parts, for example.) Indeed, suppose that the matrices $\mathbf{Y}^{(1)}, \ldots, \mathbf{Y}^{(q)}$ have i.i.d. Gaussian entries such that $\mathbf{E} Y_{j k}^{(q)}=0, \mathbf{E}\left|Y_{j k}^{(q)}\right|^{2}=1$ and

$$
\mathbf{E}\left|\operatorname{Re} Y_{j k}^{(q)}\right|^{2}=\sigma_{1}^{2}, \quad \mathbf{E}\left|\operatorname{Im} Y_{j k}^{(q)}\right|^{2}=\sigma_{2}^{2}, \quad \mathbf{E}\left(\operatorname{Re} Y_{j k}^{(q)} \operatorname{Im} X_{j k}^{(q)}\right)=\varrho \sigma_{1} \sigma_{2}
$$

for all $q=1, \ldots, m, j=1, \ldots, n_{q-1}, k=1, \ldots, n_{q}$, where $\sigma_{1}^{2}, \sigma_{2}^{2} \in[0,1]$ with $\sigma_{1}^{2}+\sigma_{2}^{2}=1$ and $\varrho \in[-1,+1]$. Then it is easy to see that the matrices $\mathbf{Y}^{(j)}$ may be represented in the form $\mathbf{Y}^{(j)}=u\left(\tau_{1} \mathbf{Y}_{j}^{\prime}+i \tau_{2} \mathbf{Y}_{j}^{\prime \prime}\right)$, where $u \in \mathbb{C},|u|=1, \tau_{1}, \tau_{2} \in[0,1], \tau_{1}^{2}+\tau_{2}^{2}=1$, and the matrices $\mathbf{Y}_{j}^{\prime}$ and $\mathbf{Y}_{j}^{\prime \prime}$ contain independent standard real Gaussian entries. Note that the factor $u$ has no influence on the singular value distribution, and, as long as it is rotationally invariant, on the eigenvalue distribution. Thus, it suffices to determine the limiting distribution for products of the matrices $\tau_{1} \mathbf{Y}_{j}^{\prime}+i \tau_{2} \mathbf{Y}_{j}^{\prime \prime}$. Note that these matrices are bi-orthogonal invariant.

Since the matrices $\tau_{1} \mathbf{Y}_{j}^{\prime}+i \tau_{2} \mathbf{Y}_{j}^{\prime \prime}$ have independent complex entries with mean 0 and variance 1, their limiting singular value distribution is well-known by the MarchenkoPastur theorem; see e.g. Theorem 3.7 in [8]. Also, from independence and bi-orthogonal invariance, it follows that for each $q=m-1, \ldots, 1$, the matrices

$$
\left(\tau_{1} \mathbf{Y}_{q}^{\prime}+i \tau_{2} \mathbf{Y}_{q}^{\prime \prime}\right)^{*}\left(\tau_{1} \mathbf{Y}_{q}^{\prime}+i \tau_{2} \mathbf{Y}_{q}^{\prime \prime}\right) \text { and }\left(\prod_{j=q+1}^{m}\left(\tau_{1} \mathbf{Y}_{j}^{\prime}+i \tau_{2} \mathbf{Y}_{j}^{\prime \prime}\right)\right)\left(\prod_{j=q+1}^{m}\left(\tau_{1} \mathbf{Y}_{j}^{\prime}+i \tau_{2} \mathbf{Y}_{j}^{\prime \prime}\right)\right)^{*}
$$

are asymptotically free. Thus, the limiting singular value distribution of the product $\prod_{j=1}^{m}\left(\tau_{1} \mathbf{Y}_{j}^{\prime}+i \tau_{2} \mathbf{Y}_{j}^{\prime \prime}\right)$ may be found by repeated application of Lemma $\mathrm{A.2}$ in the appendix. Along these lines, many of the results in Section 8 may be extended to random matrices with a more general second moment structure as in (5.19). 


\section{Stieltjes Transforms of Spectral Limits of Shifted Matrices}

In this section, we assume that $n=p$, i.e. $\mathbf{F}_{\mathbf{Y}}$ is a square matrix, and that the matrices $\mathbf{Y}^{(q)}$ have independent standard complex Gaussian entries, up to normalization.

Our aim is to describe the limit of the singular value distributions of the "shifted" matrices $\mathbf{F}_{\mathbf{Y}}-\alpha \mathbf{I}(\alpha \in \mathbb{C})$ in terms of the limit of the singular value distributions of $\mathbf{F}_{Y}$. It will be convenient to consider instead of the singular value distributions of the matrices $\mathbf{F}_{\mathbf{Y}}$ and $\mathbf{F}_{\mathbf{Y}}-\alpha \mathbf{I}$ the eigenvalue distributions of the (Hermitian) matrices

$$
\mathbf{V}=\mathbf{V}_{\mathbf{Y}}=\left[\begin{array}{cc}
\mathbf{O} & \mathbf{F}_{\mathbf{Y}} \\
\mathbf{F}_{\mathbf{Y}}^{*} & \mathbf{O}
\end{array}\right]
$$

and

$$
\mathbf{V}(\alpha)=\mathbf{V}_{\mathbf{Y}}(\alpha)=\mathbf{V}_{\mathbf{Y}}+\mathbf{J}(\alpha)
$$

where $\mathbf{J}(\alpha)$ is defined in Equation (6.1) below. More precisely, we will show that, under appropriate conditions, the mean eigenvalue distributions of the matrices $\mathbf{V}(\alpha)$ converge in moments to probability measures with compact support. Note that this implies the weak convergence of the mean eigenvalue distributions, and hence the weak convergence in probability of the eigenvalue distributions, by the variance estimate from Section A.1.

Recall the $2 n \times 2 n$ block matrix

$$
\mathbf{J}(\alpha)=\left[\begin{array}{cc}
\mathbf{O} & -\alpha \mathbf{I}_{n} \\
-\bar{\alpha} \mathbf{I}_{n} & \mathbf{O}
\end{array}\right]
$$

from the previous section. This matrix has spectral distribution $T(\alpha)=\frac{1}{2} \delta_{+|\alpha|}+\frac{1}{2} \delta_{-|\alpha|}$, where $\delta_{a}$ denotes the unit atom in the point $a$. We now calculate the $R$-transform of the distribution $T(\alpha)$. It is straightforward to check that for the distribution $T(\alpha)$, we have

$$
M_{\alpha}(z)=\frac{|\alpha|^{2} z^{2}}{1-|\alpha|^{2} z^{2}}
$$

and

$$
G_{\alpha}(z)=\frac{z}{z^{2}-|\alpha|^{2}}
$$

(Recall that $M(z)$ and $G(z)$ have been introduced above Equation (5.1).) From (6.3) it follows that

$$
G_{\alpha}^{-1}(z)=\frac{1 \pm \sqrt{1+4|\alpha|^{2} z^{2}}}{2 z} .
$$

Here we consider the principal branch of the square root. In order to obtain a function $R_{\alpha}(z)$ that is analytic at zero, we must take the plus sign. Therefore, (5.1) yields

$$
R_{\alpha}(z):=\frac{-1+\sqrt{1+4|\alpha|^{2} z^{2}}}{2 z}
$$

and

$$
\widetilde{R}_{\alpha}(z):=\frac{-1+\sqrt{1+4|\alpha|^{2} z^{2}}}{2}
$$


Remark. Similarly, we find that the $S$-transform of the distribution $T(\alpha)$ is given by

$$
S_{\alpha}(z)=\frac{1+z}{z} M_{\alpha}^{-1}(z)=\sqrt{\frac{1+z}{z}} \frac{1}{|\alpha|} .
$$

Here we take the branch of the square root such that $\operatorname{Im} S_{\alpha}(z) \geq 0$ for $z \approx 0, z \notin \mathbb{R}_{+}$.

We can now state the main result of this section.

Theorem 6.1. Assume that the mean eigenvalue distributions of the matrices $\mathbf{V}_{n}$ converge in moments (as $n \rightarrow \infty$ ) to a probability distribution $\mu_{\mathbf{V}} \neq \delta_{0}$ with compact support, with corresponding $S$-transform $S_{\mathbf{V}}(z)$. Assume also that the sequences $\mathbf{V}_{n}$ and $\mathbf{J}_{n}(\alpha)$ are asymptotically free. Then the mean eigenvalue distributions of the matrices $\mathbf{V}_{n}(\alpha)$ converge in moments (as $n \rightarrow \infty$ ) to the probability measure $\mu_{\mathbf{V}(\alpha)}:=\mu_{\mathbf{V}} \boxplus T(\alpha)$, and, for $z \in \mathbb{C}^{+}$with $|z|$ sufficiently large, the Stieltjes transform $g(z, \alpha)$ of $\mu_{\mathbf{V}(\alpha)}$ satisfies the following system of equations,

$$
\begin{aligned}
& w(z, \alpha)=z+\frac{\widetilde{R}_{\alpha}(-g(z, \alpha))}{g(z, \alpha)}, \\
& g(z, \alpha)=(1+w(z, \alpha) g(z, \alpha)) S_{\mathbf{V}}(-(1+w(z, \alpha) g(z, \alpha))) .
\end{aligned}
$$

Remark 6.2. Note that the measure $\mu_{\mathbf{V}}$ is symmetric with respect to the origin, and recall that in this case we choose the branch of $S$-transform $S_{\mathbf{V}}(z)$ as in (5.6).

Proof of Theorem [6.1. By asymptotic freeness, the matrices $\mathbf{V}(\alpha)$ converge in moments to the probability measure $\mu_{\mathbf{V}(\alpha)}:=\mu_{\mathbf{V}} \boxplus T(\alpha)$; see the remark below Definition 5.5. Let $R_{\mathbf{V}(\alpha)}$ and $\widetilde{R}_{\mathbf{V}(\alpha)}$ denote the $R$-transform and $\widetilde{R}$-transform of $\mu_{\mathbf{V}(\alpha)}$, respectively. Then, by the additivity of the $\widetilde{R}$-transform (see Eq. (5.3)), we have

$$
\widetilde{R}_{\mathbf{V}(\alpha)}(z)=\widetilde{R}_{\mathbf{V}}(z)+\widetilde{R}_{\alpha}(z) .
$$

Now we rewrite equation (6.9) in terms of Stieltjes transforms. Let $\mu$ be a symmetric probability measure on $\mathbb{R}$ with compact support, and let

$$
g_{\mu}(z):=\int \frac{1}{t-z} d \mu(t)
$$

denote the Stieltjes transform of $\mu$. It is well-known that $g_{\mu}$ maps $\mathbb{C}_{+}$to $\mathbb{C}_{+}$and that $g_{\mu}(z)=-z^{-1}+o\left(z^{-2}\right)$ as $z \rightarrow \infty$. Suppose that $z \in \mathbb{C}_{+}$with $|z|$ sufficiently large. Then, with $M_{\mu}(z)$ and $G_{\mu}(z)$ as in Section 5 , we have $g_{\mu}(z)=-G_{\mu}(z)=-\frac{1}{z}\left(1+M_{\mu}\left(\frac{1}{z}\right)\right)$. It therefore follows from (5.2) that

$$
S_{\mu}\left(-\left(1+z g_{\mu}(z)\right)\right)=\frac{g_{\mu}(z)}{1+z g_{\mu}(z)} .
$$

Using equation (5.5), we get

$$
\widetilde{R}_{\mu}^{-1}\left(-\left(1+z g_{\mu}(z)\right)\right)=-g_{\mu}(z)
$$


or

$$
\widetilde{R}_{\mu}\left(-g_{\mu}(z)\right)=-\left(1+z g_{\mu}(z)\right) .
$$

Denote by $g(z, \alpha)$ the Stieltjes transform of the limiting spectral measure of the matrices $\mathbf{V}(\alpha)$. Now replace $z$ with $-g(z, \alpha)$ in equation (6.9), for $z \in \mathbb{C}_{+}$with $|z|$ sufficiently large. We get

$$
-(1+z g(z, \alpha))=\widetilde{R}_{\mathbf{V}}(-g(z, \alpha))+\widetilde{R}_{\alpha}(-g(z, \alpha)) .
$$

We may rewrite this equation as follows

$$
-g(z, \alpha)=\widetilde{R}_{\mathbf{V}}^{-1}\left(-\left(1+g(z, \alpha)\left(z+\frac{\widetilde{R}_{\alpha}(-g(z, \alpha))}{g(z, \alpha)}\right)\right)\right) .
$$

Using the relation (5.5) again, we finally get

$$
\begin{aligned}
w & =z+\frac{\widetilde{R}_{\alpha}(-g(z, \alpha))}{g(z, \alpha)}, \\
g(z, \alpha) & =(1+w g(z, \alpha)) S_{\mathbf{V}}(-(1+w g(z, \alpha))) .
\end{aligned}
$$

Thus, Theorem 6.1 is proved completely.

Henceforward, we assume that $\mu_{\mathbf{V}}$ is a symmetric probability measure on $\mathbb{R} \backslash\{0\}$ (not necessarily with compact support). Write $S_{\mathbf{V}}(z)$ for the corresponding $S$-transform. For $\alpha \in \mathbb{C} \backslash\{0\}$, let $\mu_{\alpha}:=T(\alpha)$ and $\mu_{\mathbf{V}(\alpha)}:=\mu_{\mathbf{V}} \boxplus \mu_{\alpha}$. Write $g_{\mathbf{V}}(z), g_{\alpha}(z)$ and $g(z, \alpha):=$ $g_{\mathbf{V}(\alpha)}(z)$ for the Stieltjes transforms of $\mu_{\mathbf{V}}, \mu_{\alpha}$ and $\mu_{\mathbf{V}(\alpha)}$. Then, for $z=i y$ with $y>0$ large enough, the Stieltjes transform $g(z, \alpha)$ still satisfies the system (6.8). This follows from the proof of Theorem 6.1 if the probability measure $\mu_{\mathbf{V}}$ has bounded support, and by the approximations mentioned in Section 5 if the probability measure $\mu_{\mathbf{V}}$ has unbounded support.

In the next section, we will consider the system (6.8) with $z=i y$, where $y \geq 0$ is any non-negative real number. The next results show that this is possible under appropriate conditions.

Lemma 6.3. Let $\mu_{\mathbf{V}}, \mu_{\alpha}$ and $\mu_{\mathbf{V}(\alpha)}$ be defined as above.

(i) For any symmetric probability measure $\mu$ on $\mathbb{R}$, we have $g_{\mu}(i y) \in i \mathbb{R}^{+}$for all $y>0$.

(ii) The function $y \mapsto \operatorname{Im} g_{\alpha}($ iy $)$ is strictly increasing on $(0,|\alpha|]$ and strictly decreasing on $[|\alpha|, \infty)$, with $g_{\alpha}(i|\alpha|)=\frac{i}{2|\alpha|}$.

(iii) For all $y>0, g(i y, \alpha) \in i\left[0, \frac{1}{2|\alpha|}\right]$.

(iv) The function $z \mapsto \widetilde{R}_{\alpha}(-g(z, \alpha))$ has an analytic continuation to an open neighborhood $U$ of the upper imaginary half-axis. 
By abuse of notation, we still write $\widetilde{R}_{\alpha}(-g(z, \alpha))$ for the analytic continuation in part (iv), and we then define $w=w(z, \alpha)$ as in (6.8).

(v) For $z=i y$ with $y>0$, we have the representation

$$
\widetilde{R}_{\alpha}(-g(i y, \alpha))=-\frac{1}{2}+\frac{1}{2} \sqrt{1+4|\alpha|^{2} g(i y, \alpha)^{2}},
$$

where the branch of the square root is determined by the analytic continuation in part (iv). (Thus, the square root may be positive or negative!) In particular, $\widetilde{R}_{\alpha}(-g(i y, \alpha)) \in[-1,0]$.

(vi) Suppose that $\mu_{\mathbf{V}}$ is a probability measure on $\mathbb{R} \backslash\{0\}$. Then $1+w(i y, \alpha) g($ iy, $\alpha) \in(0,1)$ for all $y>0$, and (6.8) holds for all $z=i y$ with $y>0$.

Proof. Let us introduce some more notation. For any probability measure $\mu$ on $\mathbb{R}$, we will use the Stieltjes transform $g_{\mu}(z)$, the Cauchy transform $G_{\mu}(z)$, and the reciprocal Cauchy transform $F_{\mu}(z):=-1 / g_{\mu}(z)$. It is well-known that free additive convolution can be analyzed using subordinating functions. Let $\mathcal{F}$ denote the class of all functions $F: \mathbb{C}^{+} \rightarrow \mathbb{C}^{+}$that arise as reciprocal Cauchy transforms of probability measures on $\mathbb{R}$. Then, given $\mu_{\mathbf{V}}$ and $\mu_{\alpha}$, there exist unique functions $Z_{1}$ and $Z_{2}$ in $\mathcal{F}$ such that

$$
z=Z_{1}(z)+Z_{2}(z)-F_{\mathbf{V}(\alpha)}(z) \quad \text { and } \quad F_{\mathbf{V}(\alpha)}(z)=F_{\mathbf{V}}\left(Z_{1}(z)\right)=F_{\alpha}\left(Z_{2}(z)\right)
$$

for all $z \in \mathbb{C}^{+}$; see e.g. Chistyakov and Götze [18. It is easy to see that if the measures $\mu_{\mathbf{V}}$ and $\mu_{\alpha}$ are symmetric, $\mu_{\mathbf{V}(\alpha)}$ is also symmetric, and the functions $F_{\mathbf{V}}, F_{\alpha}, F_{\mathbf{V}(\alpha)}, Z_{1}$ and $Z_{2}$ map $i \mathbb{R}^{+}$to $i \mathbb{R}^{+}$. Finally, let us mention that for any probability measure $\mu$ on $\mathbb{R}$, we have

$$
\operatorname{Im} g_{\mu}(z) \leq \frac{1}{\operatorname{Im} z} \quad \text { and } \quad \operatorname{Im} F_{\mu}(z) \geq \operatorname{Im} z
$$

for all $z \in \mathbb{C}^{+}$, with equality only if $\mu$ is a Dirac measure.

(i) follows from a straightforward calculation.

(ii) follows by observing that

$$
\operatorname{Im} g_{\alpha}(i y)=\frac{y}{y^{2}+|\alpha|^{2}}
$$

and by using elementary calculus.

For the proof of (iii), note that (6.12) and (ii) imply

$$
\inf _{y>0} \operatorname{Im} F_{\mathbf{V}(\alpha)}(i y) \geq \inf _{y>0} \operatorname{Im} F_{\alpha}(i y) \geq 2|\alpha| .
$$

For the proof of (iv), recall that, for $z=i y$ with $y>0$ large enough,

$$
\widetilde{R}_{\alpha}(-g(z, \alpha))=\frac{1}{2}\left(-1+\sqrt{1+4|\alpha|^{2} g(z, \alpha)^{2}}\right) .
$$


Since the function $h(z):=1+4|\alpha|^{2} g(z, \alpha)^{2}$ is a non-constant analytic function, there exists a simply connected open neighborhood $U$ of the imaginary axis in $\mathbb{C}^{+}$such that $h(z) \neq 0$ for all $z \in U \backslash i \mathbb{R}^{+}$. Moreover, if $z_{0}=i y_{0}$ is a zero of $h(z)$ on the imaginary axis, it follows from (iii) that the (real-valued) function $y \mapsto h(i y)$ has a local minimum at the point $y_{0}$, and this is only possible if $z_{0}=i y_{0}$ is a zero of even order. Thus, $h(z)$ is an analytic function on $U$ such that each zero is of even order, and there exists an analytic branch of $\sqrt{h(z)}$ on $U$. Changing the sign if necessary, we may assume that $\sqrt{h(i y)} \in[0,1]$ for all sufficiently large $y$, and then the desired analytic continuation of $\widetilde{R}_{\alpha}(-g(z, \alpha))$ is given by the function $\frac{1}{2}(-1+\sqrt{h(z)})$.

This also establishes Equation (6.11). Since $h(i y)$ takes values in $[0,1]$ by part (iii), the rest of part (v) follows immediately.

We now prove (vi). It follows from our remarks around (5.11) and (5.13) that since $\mu_{\mathbf{V}}$ is a symmetric probability measure on $\mathbb{R} \backslash\{0\}, S_{\mathbf{V}}$ is analytic in an open set containing the interval $(-1,0)$. Thus, it remains to show that

$$
1+w(i y, \alpha) g(i y, \alpha) \in(0,1)
$$

for all $y>0$, for it then follows by analytic continuation that the second equation in (6.8) holds for all $y>0$.

By the definition of $w(i y, \alpha)$, we have

$$
1+w(i y, \alpha) g(i y, \alpha)=1+i y g(i y, \alpha)+\widetilde{R}_{\alpha}(-g(i y, \alpha)) .
$$

Since $\operatorname{iyg}(i y, \alpha) \in(-1,0)$ by part (i) and (6.13) and $\widetilde{R}_{\alpha}(-g(i y, \alpha)) \in[-1,0]$ by part $(\mathrm{v})$, it follows that

$$
1+w(i y, \alpha) g(i y, \alpha) \in(-1,+1)
$$

Moreover, using that

$$
\int x^{2} d \mu_{\mathbf{V}(\alpha)}(x)=\int x^{2} d \mu_{\mathbf{V}}(x)+|\alpha|^{2},
$$

it is straightforward to check that

$$
\lim _{y \rightarrow \infty} y^{2}\left(1+i y g(i y, \alpha)+\widetilde{R}_{\alpha}(-g(i y, \alpha))\right)=\int x^{2} d \mu_{\mathbf{V}}(x)>0 .
$$

Thus, we have

$$
1+w(i y, \alpha) g(i y, \alpha)>0
$$

for $y>0$ large enough, and it remains to show (by continuity) that

$$
1+w(i y, \alpha) g(i y, \alpha) \neq 0
$$

for all $y>0$. Suppose by way of contradiction that $1+w\left(i y_{0}, \alpha\right) g\left(i y_{0}, \alpha\right)=0$ for some $y_{0}>0$. By (6.15), we may assume without loss of generality that $y_{0}>0$ is maximal 
with this property. Then $1+w(i y, \alpha) g(i y, \alpha) \in(0,1)$ for all $y>y_{0}$, and by analytic continuation, the second equation in (6.8) holds for all $y>y_{0}$. Letting $y \downarrow y_{0}$, we get

$$
-g\left(i y_{0}, \alpha\right)=\lim _{y \downarrow y_{0}}\left(-(1+w(i y, \alpha) g(i y, \alpha)) S_{\mathbf{V}}(-(1+w(i y, \alpha) g(i y, \alpha)))\right)=0,
$$

since $(-x) S_{\mathbf{V}}(-x) \rightarrow 0$ as $x \downarrow 0$. But this is a contradiction to (i).

Lemma 6.4. Let $\mu_{\mathbf{V}}, \mu_{\alpha}$ and $\mu_{\mathbf{V}(\alpha)}$ be defined as above, and suppose additionally that $\mu_{\mathbf{V}}$ is a symmetric probability measure on $\mathbb{R} \backslash\{0\}$, but not a two-point distribution. Then the limits $g(0, \alpha):=\lim _{y \downarrow 0} g(i y, \alpha)$ and $(w g)(0, \alpha):=\lim _{y \downarrow 0}(w g)(i y, \alpha)$ exist for all $\alpha \neq 0$. Moreover, with the square root as in (6.11), we have

$$
\begin{aligned}
(w g)(0, \alpha) & =\frac{1}{2}\left(-1+\sqrt{1+4|\alpha|^{2} g(0, \alpha)^{2}}\right), \\
-g(0, \alpha) & =\widetilde{S}_{\mathbf{V}}(-(1+(w g)(0, \alpha)))
\end{aligned}
$$

as well as

$$
-g(0, \alpha)=\widetilde{S}_{\mathbf{V}}\left(-\frac{1}{2}\left(1+\sqrt{1+4|\alpha|^{2} g(0, \alpha)^{2}}\right)\right),
$$

where $\widetilde{S}_{\mathbf{V}}(z):=z S_{\mathbf{V}}(z)$ for $z \in(-1,0)$ and $\widetilde{S}_{\mathbf{V}}(z)$ is defined by continuous extension for $z \in\{-1,0\}$.

Proof. We proceed by contradiction. Suppose that the limit $g(0, \alpha):=\lim _{y \downarrow} g(i y, \alpha)$ does not exist. Then, by Lemma 6.3 (iii) and continuity, the set of all accumulation points is a non-degenerate closed interval $I \subset i\left[0, \frac{1}{2 \mid \alpha}\right]$. But, as a consequence of Lemma 6.3 (vi), for each accumulation point $\widetilde{g} \in I \backslash\{0\}$, we have

$$
-\widetilde{g}=\widetilde{S}_{\mathbf{V}}\left(-\frac{1}{2}\left(1+\sqrt{1+4|\alpha|^{2} \widetilde{g}^{2}}\right)\right),
$$

where the square-root can be positive or negative. It is easy to see that this implies that

$$
S_{\mathbf{V}}(z)=\sqrt{\frac{1+z}{z}} \frac{1}{|\alpha|} .
$$

In view of our remark above Theorem 6.1, this means that $\mu_{\mathbf{V}}=T(\alpha)$, in contradiction to our assumption that $\mu_{\mathbf{V}}$ is not a two-point distribution. Thus, the limit $g(0, \alpha)$ exists, and (6.17) holds.

The existence of the limit $(w g)(0, \alpha):=\lim _{y \downarrow 0}(w g)(i y, \alpha)$ as well as the relations (6.16) are now simple consequences. It is worth noting here that the sign of the square root $\sqrt{1+4|\alpha|^{2} g(i y, \alpha)^{2}}$ can only change when $g(i y, \alpha)=\frac{i}{2|\alpha|}$, and hence must be constant for $y \approx 0$ when $g(0, \alpha) \neq \frac{i}{2|\alpha|}$.

Remark 6.5. A similar argument shows that under the additional assumption that $g(i y, \alpha)$ is (jointly) continuous in $y$ and $\alpha$, we have

$$
g(0, \alpha)=\lim _{y \downarrow 0, \beta \rightarrow \alpha} g(i y, \beta)
$$

for all $\alpha \neq 0$, and the resulting function in $\alpha$ is continuous on $\mathbb{C} \backslash\{0\}$. 
Note that Equation (6.17) has the "trivial" solution $g(0, \alpha)=0$ when the sign of the square-root is negative. The next result gives a sufficient condition for $g(0, \alpha) \neq 0$.

Lemma 6.6. Let $\mu_{\mathbf{V}}, \mu_{\alpha}$ and $\mu_{\mathbf{V}(\alpha)}$ be defined as above, and suppose additionally that $\mu_{\mathbf{V}}$ is a symmetric probability measure on $\mathbb{R} \backslash\{0\}$, but not a two-point distribution. Let $\widetilde{S}_{\mathbf{V}}(z)$ be defined as in Lemma 6.4. If $\liminf _{x \uparrow 1}\left|\widetilde{S}_{\mathbf{V}}(-x)\right|>0$, then, for $\alpha \neq 0$ sufficiently close to zero, we have $g(0, \alpha) \neq 0$.

Proof. On the one hand, it is easy to see that there exists a constant $C>0$ such that

$$
\left|\widetilde{S}_{\mathbf{V}}(-r)\right| \leq C \sqrt{r}
$$

for all sufficiently small $r>0$. On the other hand, our assumption implies that

$$
\left|\widetilde{S}_{\mathbf{V}}(-1+r)\right| \geq c
$$

for all sufficiently small $r>0$.

Now fix $\alpha \neq 0$ and suppose by way of contradiction that $g(0, \alpha):=\lim _{y \rightarrow 0} g(i y, \alpha)=0$. By (6.17), (6.19) and the fact that $\operatorname{Im} \widetilde{S}_{\mathbf{V}}(z)$ is strictly negative for $z \in(-1,0)$, this implies that $\lim _{y \rightarrow 0}(1+(w g)(i y, \alpha))=0$, or (equivalently)

$$
\lim _{y \rightarrow 0}(w g)(i y, \alpha)=\lim _{y \rightarrow 0}\left(i y g(i y, \alpha)-\frac{1}{2}+\frac{1}{2} \sqrt{1+4|\alpha|^{2} g(i y, \alpha)^{2}}\right)=-1 .
$$

Thus we find that the square-root must be negative for all sufficiently small $y>0$. Using Taylor expansion, it follows that

$$
1+(w g)(i y, \alpha)=i y g(i y, \alpha)-|\alpha|^{2} g^{2}(i y, \alpha)+o\left(g^{2}(i y, \alpha)\right) \quad(y \downarrow 0) .
$$

Recalling that $1+(w g)(i y, \alpha)$ takes values in $[0,1]$, we may conclude that for all sufficiently small $y>0$,

$$
|1+(w g)(i y, \alpha)| \leq 2|\alpha|^{2} g^{2}(i y, \alpha) .
$$

By (6.8) and (6.18), it follows that for all sufficiently small $y>0$,

$$
|g(i y, \alpha)|=\left|\widetilde{S}_{\mathbf{V}}(-(1+(w g)(i y, \alpha)))\right| \leq \sqrt{2} C|\alpha||g(i y, \alpha)| .
$$

For $|\alpha|<\frac{1}{\sqrt{2} C}$, this is a contradiction. Consequently, our assumption that $g(0, \alpha)=0$ is wrong in this case, and Lemma 6.6 is proved.

\section{Density of Limiting Spectral Distribution}

In this section, we compute the density of the limit distribution of the empirical spectral distributions of the matrices $\mathbf{F}_{\mathbf{Y}}$. Here we assume that $n=p$, i.e. $\mathbf{F}_{\mathbf{Y}}$ is a square matrix, and that the matrices $\mathbf{Y}^{(q)}$ have independent standard complex Gaussian entries, up to normalization. 
To study the limiting distribution of the eigenvalue distributions of the matrices $\mathbf{F}_{\mathbf{Y}}$, we use the method of hermitization which goes back to Girko [22]. This method may be summarized as follows:

Theorem 7.1. For each $n \in \mathbb{N}$, let $\mathbf{F}_{n}$ be a random matrix of size $n \times n$ and $\mathbf{V}_{n}:=$ $\left[\begin{array}{cc}0 & \mathbf{F}_{n} \\ \mathbf{F}_{n}^{*} & 0\end{array}\right]$. Suppose that for all $\alpha \in \mathbb{C}$, the empirical spectral distributions $\nu_{n}(\cdot, \alpha)$ of the matrices $\mathbf{V}_{n}(\alpha):=\mathbf{V}_{n}+\mathbf{J}_{n}(\alpha)$ converge weakly in probability to a limit $\nu(\cdot, \alpha)$ and that the matrices $\mathbf{F}_{n}$ satisfy Conditions (C0), (C1) and (C2). Then the empirical spectral distributions of the matrices $\mathbf{F}_{n}$ converge weakly in probability to a limit $\mu_{\mathbf{F}}$, where $\mu_{\mathbf{F}}$ is the unique probability measure on the complex plane such that

$$
U_{\mathbf{F}}(\alpha):=-\int \log |z-\alpha| d \mu_{\mathbf{F}}(z)=-\int \log |x| \nu(d x, \alpha)
$$

for all $\alpha \in \mathbb{C}$.

See e.g. Lemma 4.3 in Bordenave and Chafaï [14]. Let us mention here that Conditions $(C 0),(C 1)$ and $(C 2)$ together with the assumption of weak convergence in probability imply that the function $\log |\cdot|$ is uniformly integrable in probability for the measures $\nu_{n}(\cdot, \alpha)$ and that the integrals in (17.1) are finite; see also Lemma A.9 in Appendix A.4.

Let us now suppose that the matrices $\mathbf{F}_{n}:=\mathbf{F}_{\mathbf{Y}}$ are bi-unitary invariant, that the empirical spectral distributions of the matrices $\mathbf{V}_{n}:=\left[\begin{array}{cc}0 & \mathbf{F}_{n} \\ \mathbf{F}_{n}^{*} & 0\end{array}\right]$ converge weakly in probability to a compactly supported p.m. $\mu_{\mathbf{V}}$, and that for each $k \in \mathbb{N}, \sup _{n \in \mathbb{N}} \mathbf{E} \frac{1}{n} \operatorname{Tr} \mathbf{V}_{n}^{2 k}<\infty$. Then, by the results from Section 5 , the empirical spectral distributions of the matrices $\mathbf{V}_{n}(\alpha):=\mathbf{V}_{n}+\mathbf{J}_{n}(\alpha)$ converge weakly in probability to the p.m.'s $\nu(\cdot, \alpha):=\mu_{\mathbf{V}} \boxplus T(\alpha)$, $\alpha \in \mathbb{C}$. Hence, if the matrices $\mathbf{F}_{\mathbf{Y}}$ also satisfy Conditions $(C 0),(C 1)$ and $(C 2)$, it follows from Theorem 7.1 that the empirical spectral distributions of the matrices $\mathbf{F}_{\mathbf{Y}}$ converge weakly in probability to a limit $\mu_{\mathbf{F}}$, where $\mu_{\mathbf{F}}$ is the unique probability measure on the complex plane such that

$$
U_{\mathbf{F}}(\alpha):=-\int \log |z-\alpha| d \mu_{\mathbf{F}}(z)=-\int \log |x| \nu(d x, \alpha)
$$

for all $\alpha \in \mathbb{C}$. Moreover, by Theorem 6.1, for each $\alpha \in \mathbb{C}$, the Stieltjes transform $g(z, \alpha)$ of the p.m. $\mu_{\mathbf{V}} \boxplus T(\alpha)$ satisfies the Equations (6.8).

We now describe the density $f$ of the limiting spectral distribution $\mu_{\mathbf{F}}$ in terms of the $S$-transform of the measure $\mu_{\mathbf{V}}$. In doing so, we will not use any special properties of random matrices, but only the probability measures $\mu_{\mathbf{V}}$ and $\mu_{\mathbf{F}}$ and their properties stated below.

For the rest of this section, we make the following assumptions. We assume that $\mu_{\mathbf{V}}$ is a symmetric probability measure on $\mathbb{R} \backslash\{0\}$ (not necessarily with compact support) which is not a two-point distribution. For each $\alpha \in \mathbb{C}$, let $\nu(\cdot, \alpha):=\mu_{\mathbf{V}} \boxplus T(\alpha)$, and assume that 
$\log |\cdot|$ is integrable w.r.t. $\nu(\cdot, \alpha)$ and that the corresponding Stieltjes transform $g(z, \alpha)$ satisfies the Equations (6.8) for all $z \in i \mathbb{R}^{+}$. Finally, we assume that $\mu_{\mathbf{F}}$ is a probability measure on $\mathbb{C}$ such that for all $\alpha \backslash\{0\}$,

$$
U_{\mathbf{F}}(\alpha):=-\int \log |z-\alpha| d \mu_{\mathbf{F}}(z)=-\int \log |x| \nu(d x, \alpha) .
$$

(In particular, we assume that the integrals are finite.) In the sequel, we will often write $\alpha=u+i v$, with $u, v \in \mathbb{R}$, and regard functions in the complex variable $\alpha$ as functions in the real variables $u$ and $v$.

We shall additionally make the following assumptions:

Assumption 7.2. The function $g(i y, \alpha)$ is continuous and continuously differentiable on the set $(0, \infty) \times\left(\mathbb{R}^{2} \backslash\{0\}\right)$, and the partial derivatives satisfy

$$
\frac{\partial g(i y, \alpha)}{\partial u}=\frac{2 u(-i) g(i y, \alpha)}{\sqrt{1+4|\alpha|^{2} g^{2}(i y, \alpha)}} \frac{\partial g(i y, \alpha)}{\partial y},
$$

where the square-root is the same as in (6.11). Moreover, the function $g(i y, \alpha)$ admits a continuous extension $g(0, \alpha)$ as $y \downarrow 0$.

Assumption 7.3. For any compact set $K \subset \mathbb{R}^{2} \backslash\{0\}$,

$$
\lim _{C \rightarrow \infty} \sup _{\alpha, \beta \in K} \frac{1}{|\alpha-\beta|}\left|\int_{-\infty}^{\infty} \log \left(1+\frac{y^{2}}{C^{2}}\right) \nu(d y, \alpha)-\int_{-\infty}^{\infty} \log \left(1+\frac{y^{2}}{C^{2}}\right) \nu(d y, \beta)\right|=0 .
$$

The following lemma shows that Assumptions 7.2 and 7.3 are satisfied if the probability measure $\mu_{\mathbf{V}}$ has compact support or, more generally, sufficiently small tails. Since the proof is rather technical, it is deferred to Appendix A.4

Lemma 7.4. Assumptions 7.2 and 7.3 hold for probability measures $\mu_{\mathbf{V}}$ such that $\mu_{\mathbf{V}}\left([-x,+x]^{c}\right)=\mathcal{O}\left(x^{-\eta}\right)(x \rightarrow \infty)$ for some $\eta>0$.

The logarithmic transform of the measures $\nu(\cdot \alpha)$ is defined by

$$
\Phi(\alpha):=\int_{-\infty}^{\infty} \log (|x|) \nu(d x, \alpha) \quad(\alpha \neq 0) .
$$

Note that this is exactly the integral on the right-hand side in (7.3). Similarly as above, we regard the function $\Phi$ as a function of the real parameters $u$ and $v$.

Lemma 7.5. Suppose that Assumptions 7.2 and 7.3 hold. Then the logarithmic transform $\Phi$ is differentiable on $\mathbb{R}^{2} \backslash\{0\}$ with

$$
\frac{\partial \Phi}{\partial u}(\alpha)=\frac{u}{2|\alpha|^{2}}\left(1-\sqrt{1+4|\alpha|^{2} g(0, \alpha)^{2}}\right) \quad(\alpha \neq 0),
$$

where the function $g(0, \alpha)$ and the sign of the square-root are the same as in (6.17). 
Proof. For abbreviation, set $\varkappa(y, \alpha):=(-i) g(i y, \alpha)$. Note that by Lemma 6.3 (iii), we have $\varkappa(y, \alpha) \in\left[0, \frac{1}{2|\alpha|}\right]$ for all $y \geq 0$. Throughout this proof, $\alpha \in \mathbb{R}^{2} \backslash\{0\}$ is fixed, and $h$ denotes a real number different from zero but sufficiently close to zero.

Introduce the integral

$$
B(C, \alpha)=\int_{0}^{C} \varkappa(y, \alpha) d y, \quad C>0
$$

and observe that

$$
\begin{aligned}
B(C, \alpha) & =\int_{0}^{C} \int_{-\infty}^{+\infty} \frac{y}{y^{2}+x^{2}} \nu(d x, \alpha) d y=\int_{-\infty}^{+\infty} \int_{0}^{C} \frac{y}{y^{2}+x^{2}} d y \nu(d x, \alpha) \\
& =-\int_{-\infty}^{\infty} \log (|x|) \nu(d x, \alpha)+\frac{1}{2} \int_{-\infty}^{\infty} \log \left(1+\frac{x^{2}}{C^{2}}\right) \nu(d x, \alpha)+\log C \\
& =-\Phi(\alpha)+\frac{1}{2} \int_{-\infty}^{\infty} \log \left(1+\frac{x^{2}}{C^{2}}\right) \nu(d x, \alpha)+\log C
\end{aligned}
$$

Thus,

$$
\begin{aligned}
\frac{\Phi(\alpha+h)-\Phi(\alpha)}{h} & =-\frac{B(C, \alpha+h)-B(C, \alpha)}{h} \\
& +\frac{1}{2 h}\left(\int_{-\infty}^{\infty} \log \left(1+\frac{x^{2}}{C^{2}}\right) \nu(d x, \alpha+h)-\int_{-\infty}^{\infty} \log \left(1+\frac{x^{2}}{C^{2}}\right) \nu(d x, \alpha)\right) .
\end{aligned}
$$

Clearly, by Assumption 7.3, the expression in the second line can be made arbitrarily small by choosing $C$ sufficiently large. Also, note that $\varkappa(C, \alpha) \rightarrow 0$ as $C \rightarrow \infty$. Thus, to complete the proof of the lemma, it remains to show that for any $C>0$,

$$
\frac{\partial B}{\partial u}(C, \alpha)=-\frac{u}{2|\alpha|^{2}}\left(\sqrt{1-4|\alpha|^{2} \varkappa^{2}(C, \alpha)}-\sqrt{1-4|\alpha|^{2} \varkappa^{2}(0, \alpha)}\right) .
$$

Let us mention here that the sign of the first square-root is positive for $C$ large enough, whereas the sign of the second square-root may be positive or negative, as in Lemma 6.4.

To prove (7.7), note that by Assumption 7.2, we have

$$
\begin{aligned}
\int_{c}^{C} \frac{\partial \varkappa}{\partial u}(y, \alpha) d y & =\int_{c}^{C} \frac{2 u \varkappa(y, \alpha)}{\sqrt{1-4|\alpha|^{2} \varkappa^{2}(y, \alpha)}} \frac{\partial \varkappa(y, \alpha)}{\partial y} d y \\
& =-\frac{u}{2|\alpha|^{2}}\left(\sqrt{1-4|\alpha|^{2} \varkappa^{2}(C, \alpha)}-\sqrt{1-4|\alpha|^{2} \varkappa^{2}(c, \alpha)}\right)
\end{aligned}
$$

for any $0<c<C$. It therefore follows that

$$
\begin{aligned}
& \frac{1}{h} \int_{c}^{C}(\varkappa(y, \alpha+h)-\varkappa(y, \alpha)) d y=\int_{c}^{C} \int_{0}^{1} \frac{\partial \varkappa}{\partial u}(y, \alpha+t h) d t d y=\int_{0}^{1} \int_{c}^{C} \frac{\partial \varkappa}{\partial u}(y, \alpha+t h) d y d t \\
& =\int_{0}^{1}-\frac{u+t h}{2|\alpha+t h|^{2}}\left(\sqrt{1-4|\alpha+t h|^{2} \varkappa^{2}(C, \alpha+t h)}-\sqrt{1-4|\alpha+t h|^{2} \varkappa^{2}(c, \alpha+t h)}\right) d t
\end{aligned}
$$


Thus, setting $c:=|h|$ and letting $h \rightarrow 0$, we obtain

$$
\begin{aligned}
\frac{B(C, \alpha+h)-B(C, \alpha)}{h} & =\frac{1}{h} \int_{c}^{C}(\varkappa(y, \alpha+h)-\varkappa(y, \alpha)) d y+\frac{1}{h} \int_{0}^{c}(\varkappa(y, \alpha+h)-\varkappa(y, \alpha)) d y \\
& =-\frac{u}{2|\alpha|^{2}}\left(\sqrt{1-4|\alpha|^{2} \varkappa^{2}(C, \alpha)}-\sqrt{1-4|\alpha|^{2} \varkappa^{2}(0, \alpha)}\right)+o(1),
\end{aligned}
$$

where we have used the fact that the functions $\varkappa(y, \alpha)$ and $\sqrt{1-4|\alpha|^{2} \varkappa^{2}(y, \alpha)}$ are bounded and continuous near the point $(0, \alpha)$. This completes the proof of (7.7).

Theorem 7.6. Suppose that $\mu_{\mathbf{V}}$ and $\mu_{\mathbf{F}}$ are as above and that Assumptions 7.2 and 7.3 hold. For $x>0$ and $\alpha \neq 0$, introduce the functions

$$
\varkappa(x, \alpha):=(-i) g(i x, \alpha), \quad \psi(x, \alpha):=(-i) g(i x, \alpha)(-i) w(i x, \alpha),
$$

where $w(z, \alpha)$ is defined as in Theorem 6.1, and their limits

$$
\varkappa(\alpha):=\varkappa(0, \alpha):=\lim _{x \downarrow 0} \varkappa(\alpha), \quad \psi(\alpha):=\psi(0, \alpha):=\lim _{x \downarrow 0} \psi(x, \alpha) .
$$

Then the functions $\varkappa(\cdot, \alpha)$ and $\psi(\cdot, \alpha)$ are real-valued with values in $\left[0, \frac{1}{2 \alpha}\right]$ and $[0,1]$, respectively. Furthermore, set $\xi_{\mathbf{V}}(x):=i(-x) S_{\mathbf{V}}(-x)(x \in[0 ; 1])$. Then $\xi_{\mathbf{V}} \geq 0$, and we have the relation

$$
\psi(\alpha)(1-\psi(\alpha))=|\alpha|^{2}\left(\xi_{\mathbf{V}}(1-\psi(\alpha))\right)^{2} .
$$

Alternatively, and more conveniently for applications, we may rewrite Equation (7.8) in the form of two equations:

$$
\begin{aligned}
\psi(\alpha)(1-\psi(\alpha)) & =|\alpha|^{2} \varkappa(\alpha)^{2}, \\
\varkappa(\alpha) & =\xi_{\mathbf{V}}(1-\psi(\alpha)) .
\end{aligned}
$$

Suppose additionally that there exists a finite set $A$ such that for $|\alpha| \notin A$, the function $\psi(\alpha)$ is continuously differentiable at $\alpha$. Then, on the set $\left\{(u, v) \in \mathbb{R}^{2} \backslash\{0\}: \sqrt{u^{2}+v^{2}} \notin\right.$ $A\}$, the measure $\mu_{\mathbf{F}}$ has the Lebesgue density $f$ given by

$$
f(u, v)=\frac{1}{2 \pi} \Delta \Phi(\alpha)=\frac{1}{2 \pi|\alpha|^{2}}\left(u \frac{\partial \psi}{\partial u}+v \frac{\partial \psi}{\partial v}\right) .
$$

Remark 7.7. One nuisance is that the solution to (7.9) is not unique. Indeed, the pair $(\varkappa, \psi) \equiv(0,1)$ is always a solution. However, this trivial solution can be excluded using Lemma 6.6.

In typical applications, we will proceed as follows: For given $\xi_{\mathbf{v}}$, solve the system (17.9). Using Lemma 6.6, argue that the solution is unique. This is possible at least in some cases, and notably in all our applications. Then check that the unique solution is continuously differentiable (except on a finite number of rings) and compute $f$ using (7.10). Finally, check that $f$ is indeed a probability density. 
Proof of Theorem 7.6. Note that the measure $\nu(\cdot, \alpha)$ with corresponding Stieltjes transform $g(z, \alpha)$ is symmetric to the origin. Thus, by Lemma 6.3 (iii) and (vi), we have $\varkappa(x, \alpha) \in\left[0, \frac{1}{2 \alpha}\right]$ and $\psi(x, \alpha) \in[0,1]$ for all $x \geq 0$. Also, the limits $\varkappa(0, \alpha)$ and $\psi(0, \alpha)$ exist by Lemma 6.4 and the subsequent remark. Furthermore, by our conventions concerning the $S$-transform, we have $\xi_{\mathbf{V}}(x) \geq 0$ for all $x \in(0,1)$.

We now rewrite the Equations (6.8) in terms of the real-valued functions $\varkappa, \psi$ and $\xi_{\mathbf{v}}$. Using (6.8) with $z=i x$, we have

$$
\begin{aligned}
& \psi(x, \alpha)=x \varkappa(x, \alpha)+\frac{1}{2}-\frac{1}{2} \sqrt{1-4|\alpha|^{2} \varkappa(x, \alpha)^{2}}, \\
& \varkappa(x, \alpha)=\xi_{\mathbf{v}}(1-\psi(x, \alpha))
\end{aligned}
$$

where the sign of the square-root is determined as in (6.11). Letting $x \downarrow 0$, we get

$$
\begin{aligned}
& \psi(\alpha)=\frac{1}{2}-\frac{1}{2} \sqrt{1-4|\alpha|^{2} \varkappa(\alpha)^{2}}, \\
& \varkappa(\alpha)=\xi_{\mathbf{V}}(1-\psi(\alpha)),
\end{aligned}
$$

where the sign of the square-root is determined by continuous extension. Taking squares in the first equation and rearranging terms, we deduce that

$$
\begin{aligned}
\psi(\alpha)(1-\psi(\alpha)) & =|\alpha|^{2} \varkappa(\alpha)^{2}, \\
\varkappa(\alpha) & =\xi_{\mathbf{V}}(1-\psi(\alpha)) .
\end{aligned}
$$

Eliminating $\varkappa$ from these equations leads to the equivalent equation (7.8).

Suppose additionally that there exists a finite set $A$ such that for $\alpha \notin A$, the function $\psi(\alpha)$ is continuously differentiable at $\alpha$. Let $\alpha \notin A$. By Lemma 7.5 and Equation (7.12), we have

$$
\frac{\partial \Phi(\alpha)}{\partial u}=\frac{u}{2|\alpha|^{2}}\left(1-\sqrt{1-4|\alpha|^{2} \varkappa(\alpha)^{2}}\right)=\frac{u}{|\alpha|^{2}} \psi(\alpha)
$$

Since $\psi$ is continuously differentiable w.r.t. $u$, it follows that

$$
\frac{\partial^{2} \Phi(\alpha)}{\partial u^{2}}=\frac{1}{|\alpha|^{2}} \psi-\frac{2 u^{2}}{|\alpha|^{4}} \psi+\frac{u}{|\alpha|^{2}} \frac{\partial \psi}{\partial u} .
$$

Note that all functions depend on $|\alpha|$ only, and are therefore symmetric with respect to $u$ and $v$. Thus we also have

$$
\frac{\partial^{2} \Phi(\alpha)}{\partial v^{2}}=\frac{1}{|\alpha|^{2}} \psi-\frac{2 v^{2}}{|\alpha|^{4}} \psi+\frac{v}{|\alpha|^{2}} \frac{\partial \psi}{\partial v} .
$$

Summing (7.15) and (7.16), we get, for $|\alpha| \notin A$,

$$
\Delta \Phi(\alpha)=\frac{1}{|\alpha|^{2}}\left(u \frac{\partial \psi}{\partial u}+v \frac{\partial \psi}{\partial v}\right) .
$$


Moreover, it follows from the preceding discussion that on the open set where $|\alpha| \notin A$, $\Phi(\alpha)$ is twice continuously differentiable.

In view of relation (17.3), this means that the log-potential $U_{\mathbf{F}}(\alpha)$ is twice continuously differentiable on the open set where $|\alpha| \notin A$. It therefore follows by a well known result from potential theory (see e.g. [39], Theorem II.1.3) that the restriction of $\mu_{\mathbf{F}}$ to this set is absolutely continuous with Lebesgue density

$$
f(u, v)=-\frac{1}{2 \pi} \Delta U_{\mathbf{F}}(\alpha)=\frac{1}{2 \pi} \Delta \Phi(\alpha)=\frac{1}{2 \pi|\alpha|^{2}}\left(u \frac{\partial \psi}{\partial u}+v \frac{\partial \psi}{\partial v}\right) .
$$

This completes the proof of Theorem 7.6.

Remark 7.8. Let us mention that in Chapter II.1 in [39], it is assumed that the measure $\mu$ under consideration is finite and of compact support. However, a closer inspection of the proof shows that the latter assumption may be relaxed; it is sufficient to assume that the function $z \mapsto \log ^{+}|z|$ is integrable w.r.t. $\mu$.

\section{Applications}

We consider applications of Theorems 3.2 and 4.4. These applications show that our main results allow old and new results on products of independent random matrices to be derived

in a unified way. In doing so, we shall always assume that either all random variables $X_{j k}^{(q)}$ are real with

$$
\mathbf{E} X_{j k}^{(q)}=0, \quad \mathbf{E}\left|X_{j k}^{(q)}\right|^{2}=1
$$

or all random variables $X_{j k}^{(q)}$ are complex with

$$
\mathbf{E} X_{j k}^{(q)}=0, \quad \mathbf{E}\left|\operatorname{Re} X_{j k}^{(q)}\right|^{2}=\mathbf{E}\left|\operatorname{Im} X_{j k}^{(q)}\right|^{2}=\frac{1}{2}, \quad \mathbf{E}\left(\operatorname{Re} X_{j k}^{(q)} \operatorname{Im} X_{j k}^{(q)}\right)=0 .
$$

Clearly, under these assumptions, the corresponding random variables $Y_{j k}^{(q)}$ as in (2.2) have standard real or complex Gaussian distributions, respectively, and we may use the results from the preceding sections. Let us note here that although we have stated these results only for the complex case, there exist analogous results for the real case, as mentioned at the end of Section 5. Furthermore, let us emphasize that the assumptions (8.1) and (8.2) are not needed to establish universality, but only to identify the limiting distributions. Finally, let us mention that the assumptions (8.1) and (8.2) can be relaxed a bit; see the Remark at the end of Section 5 .

\subsection{Applications of Theorem 3.2: Distribution of singular values}

In this section we consider some applications of Theorem 3.2. We start from the simplest case of Marchenko-Pastur law. 


\subsubsection{Marchenko-Pastur Law}

Let $m=1$ and let $\mathbf{X}^{(1)}=\mathbf{X}=\frac{1}{\sqrt{p}}\left(X_{j k}\right)$ be an $n \times p$ matrix. We shall assume that $p=p(n)$ and $\lim _{n \rightarrow \infty} \frac{n}{p(n)}=y \in(0,1]$. We assume that $X_{j k}, j=1, \ldots, n, k=1, \ldots, p$, are independent random variables as in $(8.1)$ or $(8.2)$. Let $\mathbb{F}(\mathbf{X}):=\mathbf{X}$, and let $\mathcal{G}_{n}(x)$ denote the empirical distribution function of the eigenvalues of the matrix $\mathbf{W}=\mathbf{F}_{\mathbf{X}} \mathbf{F}_{\mathbf{X}}^{*}=\mathbf{X X}^{*}$. Then we have the following result, cf. Marchenko and Pastur [31].

Theorem 8.1. Assume that the random variables $X_{j k}, j=1, \ldots, n, k=1, \ldots, p$ satisfy the Lindeberg condition (3.1). Then

$$
\lim _{n \rightarrow \infty} \mathcal{G}_{n}(x)=G(x) \quad \text { in probability }
$$

where $G^{\prime}(x)=\frac{\sqrt{(x-a)(b-x)}}{2 \pi x y}$ with $a=(1-\sqrt{y})^{2}, b=(1+\sqrt{y})^{2}$.

Remark 8.2. The probability distribution given by the distribution function $G(x)$ is called the Marchenko-Pastur distribution with parameter $y$.

Proof. For simplicity we shall consider only the case that the r.v.'s $X_{j k}$ are real. Let $\mathbf{Y}$ be a Gaussian matrix as in Section 11. We prove only the universality of the singular value distribution of the matrix $\mathbf{X}$, and then suppose that the limiting distribution of the singular values of the Gaussian matrix $\mathbf{Y}$ is known.

To apply Theorem [3.2, we check conditions (3.2), (3.20), (3.22), and (3.24). First we note that in our case

$$
g_{j k}^{(1)}=g_{j k}=\operatorname{Tr} \frac{\partial \mathbf{V}}{\partial X_{j k}} \mathbf{R}^{2},
$$

where $\mathbf{V}=\left[\begin{array}{cc}\mathbf{O} & \mathbf{Z} \\ \mathbf{Z}^{*} & \mathbf{O}\end{array}\right]$ and $\mathbf{Z}$ is defined as in (3.17). Let $\mathbf{e}_{1}, \ldots, \mathbf{e}_{n+p}$ be the standard orthonormal basis in $\mathbb{R}^{n+p}$. Then

$$
\frac{\partial \mathbf{V}}{\partial X_{j k}}=\mathbf{e}_{j} \mathbf{e}_{k+n}^{T}+\mathbf{e}_{k+n} \mathbf{e}_{j}^{T}, \text { for } j=1, \ldots, n ; k=1, \ldots, p .
$$

From (8.3) and (8.4) it follows that

$$
g_{j k}=\left[\mathbf{R}^{2}\right]_{j, k+n}+\left[\mathbf{R}^{2}\right]_{k+n, j} .
$$

Starting from (8.5), it is straightforward to check that condition (3.20) holds with constant $A_{0}=2 v^{-2}$, condition (3.22) holds with constant $A_{1}=8 v^{-3}$ and condition (3.24) holds with constant $A_{2}=48 v^{-4}$.

Furthermore, condition (3.2) holds with constant $C(\mathbb{F})=1$. Thus, we have checked all conditions of Theorem 3.2. By Theorem 3.2, we obtain that the limit distribution of the singular values of the matrix $\mathbf{X}$ is the same as the limit distribution of the singular values of the Gaussian matrix $\mathbf{Y}$. It is well known that the limit distribution of the spectra of 
the matrices $\mathbf{Y Y}^{*}$ is the Marchenko-Pastur distribution with parameter $y$. The density of this distribution is given by

$$
p(x)=\frac{\sqrt{(x-a)(b-x)}}{2 \pi x y} \mathbb{I}\{a \leq x \leq b\}
$$

where $a=(1-\sqrt{y})^{2}$ and $b=(1+\sqrt{y})^{2}$. Thus Theorem 8.1 is proved.

Remark 8.3. The $S$-transform of the Marchenko-Pastur distribution with parameter $y \in$ $(0,1]$, with density defined in (8.6), is given by

$$
S(z)=\frac{1}{1+y z}
$$

Proof of Remark 8.3. Let $g_{y}(z)$ denote the Stieltjes transform of the Marchenko-Pastur distribution. It is well-known that

$$
g_{y}(z)=-\frac{1}{z+y-1+y z g_{y}(z)} .
$$

See for instance [23], Equations (3.1) and (3.9). Using this equation and the formal identity

$$
g(z)=-\frac{1}{z}\left(1+M\left(\frac{1}{z}\right)\right)
$$

we get

$$
y z M^{2}(z)-(1-y z-z) M(z)+z=0 .
$$

Solving this equation with respect to $z$, we obtain

$$
M^{-1}(z)=\frac{z}{(1+y z)(1+z)} .
$$

This equality immediately implies that

$$
S(z)=\frac{1}{1+y z}
$$

and Remark 8.3 is proved.

Remark 8.4. Let $X$ be a r.v. with Marchenko-Pastur distribution with parameter $y=1$, and let $F_{t}$ denote the distribution of $(X+t)^{-1}, t \geq 0$. Then $F_{t} \rightarrow F$ in Kolmogorov distance as $t \rightarrow 0$, and the $S$-transform of the limit $X^{-1}$ is given by

$$
S(z)=-z .
$$


Proof. The first part follows from the pointwise convergence of the corresponding densities, which are easily calculated using (8.6). For the second part, we provide a formal proof. Recall that $S_{X}(z)=\frac{1}{z+1}$. The corresponding Stieltjes transform is $g_{X}(z)=\frac{1}{2}\left(-1+\sqrt{\frac{z-4}{z}}\right)$. Furthermore, we note that formally $M_{X^{-1}}(z)=z g_{X}(z)$, where $M_{X^{-1}}(z)$ denotes the generic moment generating function of the distribution of $X^{-1}$. This implies

$$
M_{X^{-1}}(z)=\frac{-z+\sqrt{z(z-4)}}{2} .
$$

From this equality it follows that

$$
M_{X^{-1}}^{-1}(z)=\frac{-z^{2}}{1+z}
$$

and therefore

$$
S_{X^{-1}}(z)=-z
$$

\subsubsection{Product of Independent Rectangular Matrices}

Let $m \geq 1$ be fixed. Let $n_{0}, \ldots, n_{m}$ denote integers depending on $n \geq 1$ such that $n_{0}=n$ and

$$
\lim _{n \rightarrow \infty} \frac{n}{n_{q}(n)}=y_{q} \in(0,1], \quad q=1, \ldots, m .
$$

Consider independent random variables $X_{j k}^{(q)}$ for $q=1, \ldots, m, j=1, \ldots, n_{q-1}, k=$ $1, \ldots, n_{q}$ as in (8.1) or (8.2). We introduce the matrices $\mathbf{X}^{(q)}=\frac{1}{\sqrt{n_{q}}}\left(X_{j k}^{(q)}\right), j=1, \ldots, n_{q-1}$, $k=1, \ldots, n_{q}$ for $q=1, \ldots, m$. Let $\mathbb{F}\left(\mathbf{X}^{(1)}, \ldots, \mathbf{X}^{(m)}\right)=\prod_{q=1}^{m} \mathbf{X}^{(q)}, \mathbf{F}=\mathbf{F}_{\mathbf{X}}$ and $\mathbf{W}=$ $\mathbf{F F}^{*}$. Denote by $\mathcal{G}_{n}(x)$ the empirical spectral distribution function of matrix $\mathbf{W}$. Then we have the following result, see also Müller [33] and Burda, Janik, Waclaw [15] for the Gaussian case and Alexeev, Götze, Tikhomirov [3], 4, [5] and Tikhomirov [43] for the general case.

Theorem 8.5. Assume that the random variables $X_{j k}^{(q)}$, for $q=1, \ldots, m$ and $j=$ $1, \ldots, n_{q-1} ; k=1, \ldots, n_{q}$, satisfy the Lindeberg condition (3.1). Then

$$
\lim _{n \rightarrow \infty} \mathcal{G}_{n}(x)=G^{(m)}(x) \quad \text { in probability }
$$

where the Stieltjes transform $s(z)=\int_{-\infty}^{\infty} \frac{1}{x-z} d G^{(m)}(x)$ of the distribution function $G^{(m)}(x)$ is determined by the equation

$$
1+z s(z)-s(z) \prod_{q=1}^{m}\left(1-y_{q}-y_{q} z s(z)\right)=0 .
$$


Proof. For simplicity we shall assume that all r.v.'s $X_{j k}^{(q)}$ are real. Let $\mathbf{Y}^{(1)}, \ldots, \mathbf{Y}^{(q)}$ be Gaussian matrices as in Section 1

We now check that the function $\mathbb{F}$ satisfies conditions (3.2), (3.20), (3.22) and (3.24). Condition (3.2) follows from the obvious inequality

$$
\operatorname{rank}\left\{\prod_{q=1}^{m} \mathbf{A}^{(q)}-\prod_{q=1}^{m} \mathbf{B}^{(q)}\right\} \leq \sum_{q=1}^{m} \operatorname{rank}\left\{\mathbf{A}^{(q)}-\mathbf{B}^{(q)}\right\} .
$$

Let $\mathbf{Z}^{(1)}, \ldots, \mathbf{Z}^{(q)}$ be defined as in (3.17). Furthermore, introduce the matrices

$$
\begin{gathered}
\mathbf{H}^{(q)}=\left[\begin{array}{cc}
\mathbf{Z}^{(q)} & \mathbf{O} \\
\mathbf{O} & \left(\mathbf{Z}^{(m-q+1)}\right)^{*}
\end{array}\right], \quad \mathbf{V}_{a, b}=\prod_{q=a}^{b} \mathbf{H}^{(q)}, \\
\mathbf{J}(\alpha)=\left[\begin{array}{cc}
\mathbf{O} & -\alpha \mathbf{I}_{n_{0}} \\
-\bar{\alpha} \mathbf{I}_{n_{m}} & \mathbf{O}
\end{array}\right], \quad \mathbf{J}=\mathbf{J}(-1) .
\end{gathered}
$$

Using these notations we have

$$
\mathbf{V}=\mathbf{V}(\varphi)=\mathbf{V}_{1, m} \mathbf{J}
$$

and

$$
\frac{\partial \mathbf{V}_{1 m}}{\partial Z_{j k}^{(q)}}=\frac{1}{1+\delta_{q, m-q+1}}\left(\mathbf{V}_{1, q-1} \frac{\partial \mathbf{H}^{(q)}}{\partial Z_{j k}^{(q)}} \mathbf{V}_{q+1, m}+\mathbf{V}_{1, m-q} \frac{\partial \mathbf{H}^{(m-q+1)}}{\partial Z_{j k}^{(q)}} \mathbf{V}_{m-q+2, m}\right) .
$$

For $q=1, \ldots, m+1$, let $\mathbf{e}_{j}^{(q)}, j=1, \ldots, n_{q-1}+n_{m-q+1}$, denote the standard orthogonal basis in $\mathbb{R}^{n_{q-1}+n_{m-q+1}}$. Then

$$
\frac{\partial \mathbf{V}_{1 m}}{\partial Z_{j k}^{(q)}}=\mathbf{V}_{1, q-1} \mathbf{e}_{j}^{(q)}\left(\mathbf{e}_{k}^{(q+1)}\right)^{T} \mathbf{V}_{q+1, m}+\mathbf{V}_{1, m-q} \mathbf{e}_{k+n_{m-q}}^{(m-q+1)}\left(\mathbf{e}_{j+n_{m-q+1}}^{(m-q+2)}\right)^{T} \mathbf{V}_{m-q+2, m}
$$

for $j=1, \ldots, n_{q-1} ; k=1, \ldots, n_{q}$. From here it follows that

$g_{j k}^{(q)}=\operatorname{Tr}\left(\left(\mathbf{V}_{1, q-1} \mathbf{e}_{j}^{(q)}\left(\mathbf{e}_{k}^{(q+1)}\right)^{T} \mathbf{V}_{q+1, m}+\mathbf{V}_{1, m-q} \mathbf{e}_{k+n_{m-q}}^{(m-q+1)}\left(\mathbf{e}_{j+n_{m-q+1}}^{(m-q+2)}\right)^{T} \mathbf{V}_{m-q+2, m}\right) \mathbf{J R}^{2}\right)$.

Consider for instance the first term in the right hand side of (8.9). We have

$$
\left|\operatorname{Tr}\left(\mathbf{V}_{1, q-1} \mathbf{e}_{j}^{(q)}\left(\mathbf{e}_{k}^{(q+1)}\right)^{T} \mathbf{V}_{q+1, m} \mathbf{J R}^{2}\right)\right| \leq v^{-2}\left\|\mathbf{V}_{1, q-1} \mathbf{e}_{j}^{(q)}\right\|_{2}\left\|\left(\mathbf{e}_{k}^{(q+1)}\right)^{T} \mathbf{V}_{q+1, m}\right\|_{2} .
$$

Note that for each $q=1, \ldots, m$, the vector $\mathbf{V}_{1, q-1} \mathbf{e}_{j}^{(q)}$ is independent of the matrix $\mathbf{Z}^{(q)}$ due to the block structure of the matrices $\mathbf{H}^{(q)}$. From here it follows that

$$
\mathbf{E}\left\{\left\|\mathbf{V}_{1, q-1} \mathbf{e}_{j}^{(q)}\right\|_{2}^{2} \mid X_{j k}^{(q)}, Y_{j k}^{(q)}\right\}=\mathbf{E}\left\|\mathbf{V}_{1, q-1} \mathbf{e}_{j}^{(q)}\right\|_{2}^{2}
$$


and by Lemma 5.1 in the Appendix of [4], we have

$$
\mathbf{E}\left\{\left\|\mathbf{V}_{1, q-1} \mathbf{e}_{j}^{(q)}\right\|_{2}^{2} \mid X_{j k}^{(q)}, Y_{j k}^{(q)}\right\} \leq C .
$$

Similarly we get

$$
\mathbf{E}\left\{\left(\| \mathbf{e}_{k}^{(q+1)}\right)^{T} \mathbf{V}_{q+1, m} \|_{2}^{2} \mid X_{j k}^{(q)}, Y_{j k}^{(q)}\right\} \leq C,
$$

$q=1, \ldots, m$. Combining these estimates, it follows that

$$
\mathbf{E}\left\{\mid g_{j k}^{(q)} \| X_{j k}^{(q)}, Y_{j k}^{(q)}\right\} \leq C v^{-2} .
$$

Furthermore, it is straightforward to check that

$$
\frac{\partial^{2} \mathbf{V}}{\partial\left(Z_{j k}^{(q)}\right)^{2}}=\mathbf{O}
$$

This implies that

$$
\frac{\partial g_{j k}^{(q)}}{\partial Z_{j k}^{(q)}}=-\operatorname{Tr} \frac{\partial \mathbf{V}}{\partial Z_{j k}^{(q)}} \mathbf{R}^{2} \frac{\partial \mathbf{V}}{\partial Z_{j k}^{(q)}} \mathbf{R}-\operatorname{Tr} \frac{\partial \mathbf{V}}{\partial Z_{j k}^{(q)}} \mathbf{R} \frac{\partial \mathbf{V}}{\partial Z_{j k}^{(q)}} \mathbf{R}^{2}
$$

Using equalities (8.12), (8.8), (8.10) and Lemma 5.1 in the Appendix of [4], we get

$$
\mathbf{E}\left\{\left|\frac{\partial g_{j k}^{(q)}}{\partial Z_{j k}^{(q)}}\right| \mid X_{j k}^{(q)}, Y_{j k}^{(q)}\right\} \leq C v^{-3} .
$$

Furthermore,

$$
\begin{aligned}
\frac{\partial^{2} g_{j k}^{(q)}}{\partial\left(Z_{j k}^{(q)}\right)^{2}}=2 \operatorname{Tr} & \frac{\partial \mathbf{V}}{\partial Z_{j k}^{(q)}} \mathbf{R}^{2} \frac{\partial \mathbf{V}}{\partial Z_{j k}^{(q)}} \mathbf{R} \frac{\partial \mathbf{V}}{\partial Z_{j k}^{(q)}} \mathbf{R} \\
& +2 \operatorname{Tr} \frac{\partial \mathbf{V}}{\partial Z_{j k}^{(q)}} \mathbf{R} \frac{\partial \mathbf{V}}{\partial Z_{j k}^{(q)}} \mathbf{R}^{2} \frac{\partial \mathbf{V}}{\partial Z_{j k}^{(q)}} \mathbf{R}+2 \operatorname{Tr} \frac{\partial \mathbf{V}}{\partial Z_{j k}^{(q)}} \mathbf{R} \frac{\partial \mathbf{V}}{\partial Z_{j k}^{(q)}} \mathbf{R} \frac{\partial \mathbf{V}}{\partial Z_{j k}^{(q)}} \mathbf{R}^{2} .
\end{aligned}
$$

Using equalities (8.13), (8.8), (8.10) and Lemma 5.2 in the Appendix of [4], it is straightforward to prove that

$$
\mathbf{E}\left\{\mid \frac{\partial^{2} g_{j k}^{(q)}}{\partial\left(Z_{j k}^{(q)}\right)^{2}} \| X_{j k}^{(q)}, Y_{j k}^{(q)}\right\} \leq C v^{-4}
$$

Inequalities (8.11), (8.12), (8.14) imply that conditions (3.20), (3.22), (3.24) hold. Thus, from Theorem 3.2, it follows that the limit distribution of the singular values of the matrices $\mathbf{F}_{\mathbf{X}}$ is the same as the limit distribution of the singular values of the matrices $\mathbf{F}_{\mathbf{Y}}$. For the Gaussian case we may prove that the random matrices $\left(\prod_{q=1}^{l-1} \mathbf{Y}^{(q)}\right)^{*}\left(\prod_{q=1}^{l-1} \mathbf{Y}^{(q)}\right)$ and $\mathbf{Y}^{(l)} \mathbf{Y}^{(l)^{*}}$ are asymptotically free for any $l=1, \ldots, m$. For details see [4], Lemma 4.1. 
From here and Lemma A.2 it follows that the $S$-transform of the distribution function $G^{(m)}(x)$ is given by

$$
S(z)=\prod_{q=1}^{m} \frac{1}{1+y_{q} z} .
$$

The last relation implies that

$$
1+z s(z)-s(z) \prod_{q=1}^{m}\left(1-y_{q}-y_{q} z s(z)\right)=0 .
$$

For details, see Equations (4.9) and (4.13) in [4]. Thus Theorem 8.5 is proved.

Corollary 8.6. Assume that the conditions of Theorem 8.5 hold and $y_{1}=\cdots=y_{m}=1$. Then

$$
\lim _{n \rightarrow \infty} \mathcal{G}_{n}(x)=G(x) \text { in probability }
$$

where the Stieltjes transform of $G(x)$ is determined by the equation

$$
1+z s(z)+(-1)^{m+1} z^{m} s^{m+1}(z)=0 .
$$

\subsubsection{Powers of Random Matrices}

Consider an $n \times n$ random matrix $\mathbf{X}=\frac{1}{\sqrt{n}}\left(X_{j k}\right)$ with independent entries $X_{j k}$ as in (8.1) or (8.2). We shall assume that the Lindeberg condition (3.1) holds. For fixed $m \geq 1$, consider the function $\mathbb{F}(\mathbf{X})=\mathbf{X}^{m}$. Let $\mathbf{F}=\mathbf{F}_{\mathbf{X}}$ and $\mathbf{W}=\mathbf{F F}^{*}$, and denote by $\mathcal{G}_{n}(x)$ the distribution function of the eigenvalues of the matrix $\mathbf{W}$. Then we have the following result, cf. Alexeev, Götze and Tikhomirov [2, [3].

Theorem 8.7. Assume that the random variables $X_{j k}$ satisfy the Lindeberg condition (3.1). Then

$$
\lim _{n \rightarrow \infty} \mathcal{G}_{n}(x)=G^{(m)}(x) \quad \text { in probability }
$$

where $G^{(m)}(x)$ is defined by its Stieltjes transform $s(z)$, which satisfies the equation

$$
1+z s(z)+(-1)^{m+1} z^{m} s^{m+1}(z)=0,
$$

or its moments

$$
M_{k}=\int_{0}^{\infty} x^{k} d G^{(m)}(x)=\frac{1}{m k+1}\left(\begin{array}{c}
(m+1) k \\
k
\end{array}\right) .
$$

Remark 8.8. The numbers $M_{k}$ appearing in (8.17) are called Fuss-Catalan numbers.

Proof. Again, for simplicity we shall consider only the case that the r.v.'s $X_{j k}$ are real. Let $\mathbf{Y}$ be a Gaussian matrix as in Section 1 .

We start by noting that the rank condition (3.2) holds with constant $C(\mathbb{F})=m$. In fact,

$$
\operatorname{rank}\left\{\mathbf{A}^{m}-\mathbf{B}^{m}\right\} \leq m \operatorname{rank}\{\mathbf{A}-\mathbf{B}\}
$$


In this case we have

$$
g_{j k}=\sum_{q=1}^{m} \operatorname{Tr}\left(\mathbf{H}^{q-1} \frac{\partial \mathbf{H}}{\partial X_{j k}} \mathbf{H}^{m-q} \mathbf{J R}^{2}\right)
$$

where $\mathbf{H}=\left[\begin{array}{cc}\mathbf{Z} & \mathbf{O} \\ \mathbf{O} & \mathbf{Z}^{*}\end{array}\right]$ and $\mathbf{J}=\mathbf{J}(-1)$. Clearly,

$$
\frac{\partial \mathbf{H}}{\partial X_{j k}}=\mathbf{e}_{j} \mathbf{e}_{k}^{T}+\mathbf{e}_{k+n} \mathbf{e}_{j+n}^{T}=: \boldsymbol{\Delta}_{j k} .
$$

Using this notation we may write

$$
\begin{aligned}
g_{j k} & =\sum_{q=1}^{m} \operatorname{Tr}\left(\boldsymbol{\Delta}_{j k} \mathbf{H}^{m-q} \mathbf{J R}^{2} \mathbf{H}^{q-1}\right) \\
& =\sum_{q=1}^{m} \mathbf{e}_{k}^{T} \mathbf{H}^{m-q} \mathbf{J} \mathbf{R}^{2} \mathbf{H}^{q-1} \mathbf{e}_{j}+\sum_{q=1}^{m} \mathbf{e}_{j+n}^{T} \mathbf{H}^{m-q} \mathbf{J R}^{2} \mathbf{H}^{q-1} \mathbf{e}_{k+n} .
\end{aligned}
$$

Consider for instance the first sum on the right-hand side. Applying Hölder's inequality, we get

$$
\left|g_{j k}\right| \leq \sum_{q=1}^{m}\left\|\mathbf{e}_{k}^{T} \mathbf{H}^{m-q}\right\|_{2}\left\|\mathbf{J R}^{2} \mathbf{H}^{q-1} \mathbf{e}_{j}\right\|_{2} \leq v^{-2}\left\|\mathbf{e}_{k}^{T} \mathbf{H}^{m-q}\right\|_{2}\left\|\mathbf{H}^{q-1} \mathbf{e}_{j}\right\|_{2} .
$$

Furthermore, we note that

$$
\mathbf{H}=\mathbf{H}^{(j, k)}+\frac{Z_{j k}}{\sqrt{n}} \boldsymbol{\Delta}_{j k},
$$

where $\mathbf{H}^{(j, k)}$ is obtained from $\mathbf{H}$ by replacing the entry $Z_{j k}$ with zero. Note that, for $q \geq 2$,

$$
\boldsymbol{\Delta}_{j k}^{q}= \begin{cases}\boldsymbol{\Delta}_{j k}, & \text { for } j=k, \\ \mathbf{O}, & \text { for } j \neq k .\end{cases}
$$

We shall use the representation

$$
\mathbf{H}^{q}=\left(\mathbf{H}^{(j, k)}\right)^{q}+\sum_{s=1}^{q}\left(\frac{Z_{j k}}{\sqrt{n}}\right)^{s} \sum_{\substack{m_{1}, \ldots, m_{q-s} \geq 0: \\ m_{1}+\cdots+m_{q-s} \leq q-s}}\left(\mathbf{H}^{(j, k)}\right)^{m_{1}} \boldsymbol{\Delta}_{j k} \cdots\left(\mathbf{H}^{(j, k)}\right)^{m_{q-s}} .
$$

By the independence of the matrices $\mathbf{H}^{(j, k)}$ and the random variables $Z_{j k}$, we have

$$
\begin{aligned}
& \left|\mathbf{E}\left\{\left\|\mathbf{e}_{k}^{T} \mathbf{H}^{m-q}\right\|_{2}^{2} \mid X_{j k}, Y_{j k}\right\}\right| \leq C_{1} \mathbf{E}\left\|\mathbf{e}_{k}^{T}\left(\mathbf{H}^{(j, k)}\right)^{m-q}\right\|_{2}^{2} \\
& +C_{2} \sum_{s=1}^{m-q} \sum_{\substack{m_{1}, \ldots, m_{m-q-s} \geq 0: \\
m_{1}+\cdots+m_{q-s} \leq m-q-s}} \mathbf{E}\left\|\mathbf{e}_{k}^{T}\left(\mathbf{H}^{(j, k)}\right)^{m_{1}} \boldsymbol{\Delta}_{j k} \cdots\left(\mathbf{H}^{(j, k)}\right)^{m_{m-q-s}}\right\|_{2}^{2},
\end{aligned}
$$


for some absolute positive constants $C_{1}, C_{2}$. Similarly we have

$$
\begin{aligned}
& \left|\mathbf{E}\left\{\left\|\mathbf{H}^{q-1} \mathbf{e}_{j}\right\|_{2}^{2} \mid X_{j k}, Y_{j k}\right\}\right| \leq C_{1} \mathbf{E}\left\|\left(\mathbf{H}^{(j, k)}\right)^{q-1} \mathbf{e}_{j}\right\|_{2}^{2} \\
+ & C_{2} \sum_{s=1}^{q-1} \sum_{\substack{m_{1}, \ldots, m_{q-1-s} \geq 0: \\
m_{1}+\cdots+m_{q-1-s} \leq q-1-s}} \mathbf{E}\left\|\left(\mathbf{H}^{(j, k)}\right)^{m_{1}} \boldsymbol{\Delta}_{j k} \cdots\left(\mathbf{H}^{(j, k)}\right)^{m_{q-1-s}} \mathbf{e}_{j}\right\|_{2}^{2} .
\end{aligned}
$$

By Lemma 3 in [42, we get

$$
\mathbf{E}\left\{\left|g_{j k}\right| \mid X_{j k}, Y_{j k}\right\} \leq C,
$$

for some positive constant $C>0$. (Let us note here that the moment conditions here are a bit different from those in [42, but using (3.9) - (3.12), it is easy to see that the conclusion still holds.) Furthermore,

$$
\frac{\partial g_{j k}}{\partial Z_{j k}}=-\operatorname{Tr} \frac{\partial \mathbf{H}^{m}}{\partial Z_{j k}} \mathbf{J R}^{2} \frac{\partial \mathbf{H}^{m}}{\partial Z_{j k}} \mathbf{J R}-\operatorname{Tr} \frac{\partial \mathbf{H}^{m}}{\partial Z_{j k}} \mathbf{J R} \frac{\partial \mathbf{H}^{m}}{\partial Z_{j k}} \mathbf{J R}^{2}+\operatorname{Tr} \frac{\partial^{2} \mathbf{H}^{m}}{\partial Z_{j k}^{2}} \mathbf{J R}^{2} .
$$

We have

$$
\begin{aligned}
\frac{\partial^{2} \mathbf{H}^{m}}{\partial Z_{j k}^{2}} & =\sum_{q=1}^{m} \sum_{s=1}^{q-1} \mathbf{H}^{s-1} \frac{\partial \mathbf{H}}{\partial Z_{j k}} \mathbf{H}^{q-1-s} \frac{\partial \mathbf{H}}{\partial Z_{j k}} \mathbf{H}^{m-q}+\sum_{q=1}^{m} \sum_{s=1}^{m-q} \mathbf{H}^{q-1} \frac{\partial \mathbf{H}}{\partial Z_{j k}} \mathbf{H}^{s-1} \frac{\partial \mathbf{H}}{\partial Z_{j k}} \mathbf{H}^{m-q-s} \\
& =\sum_{q=1}^{m} \sum_{s=1}^{q-1} \mathbf{H}^{s-1} \boldsymbol{\Delta}_{j k} \mathbf{H}^{q-1-s} \boldsymbol{\Delta}_{j k} \mathbf{H}^{m-q}+\sum_{q=1}^{m} \sum_{s=1}^{m-q} \mathbf{H}^{q-1} \boldsymbol{\Delta}_{j k} \mathbf{H}^{s-1} \boldsymbol{\Delta}_{j k} \mathbf{H}^{m-q-s} .
\end{aligned}
$$

Thus, we may rewrite the equality (8.19) in the form

$$
\begin{aligned}
\frac{\partial g_{j k}}{\partial Z_{j k}}= & -\sum_{q=1}^{m} \sum_{r=1}^{m} \operatorname{Tr} \mathbf{H}^{q-1} \boldsymbol{\Delta}_{j k} \mathbf{H}^{m-q} \mathbf{J R}^{2} \mathbf{H}^{r-1} \boldsymbol{\Delta}_{j k} \mathbf{H}^{m-r} \mathbf{J R} \\
& -\sum_{q=1}^{m} \sum_{r=1}^{m} \operatorname{Tr} \mathbf{H}^{q-1} \boldsymbol{\Delta}_{j k} \mathbf{H}^{m-q} \mathbf{J} \mathbf{R} \mathbf{H}^{r-1} \boldsymbol{\Delta}_{j k} \mathbf{H}^{m-r} \mathbf{J R}^{2} \\
& +\sum_{q=1}^{m} \sum_{s=1}^{q-1} \operatorname{Tr} \mathbf{H}^{s-1} \boldsymbol{\Delta}_{j k} \mathbf{H}^{q-1-s} \boldsymbol{\Delta}_{j k} \mathbf{H}^{m-q} \mathbf{J R}^{2} \\
& +\sum_{q=1}^{m} \sum_{s=1}^{m-q} \operatorname{Tr} \mathbf{H}^{q-1} \boldsymbol{\Delta}_{j k} \mathbf{H}^{s-1} \boldsymbol{\Delta}_{j k} \mathbf{H}^{m-q-s} \mathbf{J R}^{2} .
\end{aligned}
$$

All summands on the r.h.s of (8.20) may be bounded similarly. For instance,

$$
\begin{aligned}
\mathbf{E}\left\{\mid \operatorname{Tr} \mathbf{H}^{q-1} \boldsymbol{\Delta}_{j k} \mathbf{H}^{m-q} \mathbf{J R}^{2} \mathbf{H}^{r-1} \boldsymbol{\Delta}_{j k} \mathbf{H}^{m-r} \mathbf{J R} \| X_{j k}, Y_{j k}\right\} \\
\leq v^{-3} \sum_{t, u, v, w} \mathbf{E}\left\{\left\|\mathbf{e}_{t}^{T} \mathbf{H}^{m-q}\right\|_{2}\left\|\mathbf{H}^{r-1} \mathbf{e}_{u}\right\|_{2}\left\|\mathbf{e}_{v}^{T} \mathbf{H}_{m-r}\right\|_{2}\left\|\mathbf{H}^{q-1} \mathbf{e}_{w}\right\|_{2} \mid X_{j k}, Y_{j k}\right\},
\end{aligned}
$$


where the sum is taken over all $t, u, v, w$ from the set $\{j, k, j+n, k+n\}$. Applying Hölder's inequality, the representation (8.18) and Lemmas 5.2 and 5.4 in [5], we get

$$
\mathbf{E}\left\{\mid \operatorname{Tr} \mathbf{H}^{(q-1)} \boldsymbol{\Delta}_{j k} \mathbf{H}^{m-q} \mathbf{J R}^{2} \mathbf{H}^{r-1} \boldsymbol{\Delta}_{j k} \mathbf{H}^{m-r} \mathbf{J R} \| X_{j k}, Y_{j k}\right\} \leq C .
$$

Thus we can prove that

$$
\mathbf{E}\left\{\left|\frac{\partial g_{j k}}{\partial Z_{j k}}\right| \mid X_{j k}, Y_{j k}\right\} \leq C v^{-3}
$$

which means that condition (3.22) holds.

Consider now

$$
\begin{aligned}
\frac{\partial^{2} g_{j k}}{\partial Z_{j k}^{2}} & =\operatorname{Tr} \frac{\partial^{3} \mathbf{H}^{q}}{\partial Z_{j k}^{3}} \mathbf{R}^{2}-3 \operatorname{Tr} \frac{\partial^{2} \mathbf{H}^{q}}{\partial Z_{j k}^{2}} \mathbf{R}^{2} \frac{\partial \mathbf{H}^{q}}{\partial Z_{j k}} \mathbf{R}-3 \operatorname{Tr} \frac{\partial^{2} \mathbf{H}^{q}}{\partial Z_{j k}^{2}} \mathbf{R} \frac{\partial \mathbf{H}^{q}}{\partial Z_{j k}} \mathbf{R}^{2} \\
& +2 \operatorname{Tr} \frac{\partial \mathbf{H}^{q}}{\partial Z_{j k}} \mathbf{R}^{2} \frac{\partial \mathbf{H}^{q}}{\partial Z_{j k}} \mathbf{R} \frac{\partial \mathbf{H}^{q}}{\partial Z_{j k}} \mathbf{R}+2 \operatorname{Tr} \frac{\partial \mathbf{H}^{q}}{\partial Z_{j k}} \mathbf{R} \frac{\partial \mathbf{H}^{q}}{\partial Z_{j k}} \mathbf{R}^{2} \frac{\partial \mathbf{H}^{q}}{\partial Z_{j k}} \\
& +2 \operatorname{Tr} \frac{\partial \mathbf{H}^{q}}{\partial Z_{j k}} \mathbf{R} \frac{\partial \mathbf{H}^{q}}{\partial Z_{j k}} \mathbf{R} \frac{\partial \mathbf{H}^{q}}{\partial Z_{j k}} \mathbf{R}^{2} .
\end{aligned}
$$

Similarly to inequality (8.21) we get

$$
\mathbf{E}\left\{\left|\frac{\partial^{2} g_{j k}}{\partial Z_{j k}^{2}}(\theta)\right| \mid X_{j k}, Y_{j k}\right\} \leq C v^{-4} .
$$

Thus condition (3.24) is proved.

As follows from Theorem 3.2 the limiting singular value distributions of the matrices $\mathbf{F}_{\mathbf{X}}$ and $\mathbf{F}_{\mathbf{Y}}$ are the same. In the Gaussian case the limit distribution is computed in Section 4 of $[5]$.

Remark 8.9. It follows from equation (8.16) that the $S$-transform of the distribution $G^{(m)}(x)$ is given by the formula

$$
S(z)=\frac{1}{(1+z)^{m}}
$$

See equality (8.15) for $y_{1}=\cdots=y_{m}=1$ as well.

\subsubsection{Product of Powers of Independent Matrices}

Consider independent random $n \times n$ matrices $\mathbf{X}^{(1)}, \ldots, \mathbf{X}^{(m)}$ with independent entries $\frac{1}{\sqrt{n}} X_{j k}^{(q)}, q=1, \ldots, m, j, k=1, \ldots, n$. We shall assume that (8.1) or (8.2) holds. Let $m_{1}, \ldots, m_{m}$ be fixed positive integers. Let $\mathbb{F}\left(\mathbf{X}^{(1)}, \ldots, \mathbf{X}^{(q)}\right)=\prod_{q=1}^{m}\left(\mathbf{X}^{(q)}\right)^{m_{q}}$, and let $\mathcal{G}_{n}(x)$ denote the empirical distribution function of the eigenvalues of matrix $\mathbf{W}_{\mathbf{X}}=$ $\mathbf{F}_{\mathbf{X}} \mathbf{F}_{\mathbf{X}}^{*}$. Then we have the following result, cf. Timushev and Tikhomirov [42]. 
Theorem 8.10. Assume that the random variables $X_{j k}^{(q)}$, for $q=1, \ldots, m$ and $j, k=$ $1, \ldots, n$, satisfy the Lindeberg condition (3.1). Then

$$
\lim _{n \rightarrow \infty} \mathcal{G}_{n}(x)=G^{(m)}(x) \quad \text { in probability }
$$

where the Stieltjes transform $s(z)=\int_{-\infty}^{\infty} \frac{1}{x-z} d G^{(m)}(x)$ of the distribution function $G^{(m)}(x)$ is determined by the equation

$$
1+z s(z)+(-1)^{k+1} z^{k} s^{k+1}(z)=0
$$

where $k=m_{1}+\cdots+m_{m}$.

Proof. For simplicity we shall assume that the $X_{j k}^{(q)}$ are real. Let $\mathbf{Y}^{(1)}, \ldots, \mathbf{Y}^{(m)}$ denote the corresponding Gaussian matrices. We shall apply Theorem 3.2. First we note that

$$
\operatorname{rank}\left\{\mathbf{F}_{\mathbf{X}}-\mathbf{F}_{\widehat{\mathbf{X}}}\right\} \leq \sum_{q=1}^{m} m_{q} \operatorname{rank}\left\{\mathbf{X}^{(q)}-\widehat{\mathbf{X}}^{(q)}\right\}
$$

This implies condition (3.2). Conditions (3.20), (3.22), (3.24) may be checked similarly as in Subsections 8.1.2 and 8.1.3. For more details see [42].

Theorem 3.2 now implies that the limit distributions of the singular values of the matrices $\mathbf{F}_{\mathbf{X}}$ and $\mathbf{F}_{\mathbf{Y}}$ are the same. For the Gaussian case we use the asymptotic freeness of the matrices $\left(\prod_{q=l}^{m}\left(\mathbf{Y}^{(l)}\right)^{m_{l}}\right)\left(\prod_{q=l}^{m}\left(\mathbf{X}^{(l)}\right)^{m_{l}}\right)^{*}$ and $\left.\left(\left(\mathbf{Y}^{(l-1)}\right)^{m_{l-1}}\right)^{*}\left(\mathbf{Y}^{(l-1)}\right)^{m_{l-1}}\right)$ for $l=$ $2, \ldots, m$. The proof of this claim repeats the proof of Lemma 4.2 in [5]. From here it follows that the $S$-transform of the distribution of $\mathcal{G}_{n}(x)$ is given by

$$
S(z)=\prod_{q=1}^{m} \frac{1}{(1+z)^{m_{q}}}=\frac{1}{(1+z)^{k}} .
$$

This completes the proof of Theorem 8.10 ,

\subsubsection{Polynomials of Random Matrices}

Let $\mathbf{X}^{(1)}, \ldots, \mathbf{X}^{(m)}$ be independent $n \times n$ random matrices with the entries $\frac{1}{\sqrt{n}} X_{j k}^{(q)}$, where the r.v.'s $X_{j k}^{(q)}$ are independent random variables as in (8.1) or (8.2). Consider the matrixvalued function $\mathbb{F}\left(\mathbf{X}^{(1)}, \ldots, \mathbf{X}^{(m)}\right)=\sum_{q=1}^{m} \sum_{1 \leq i_{1}, \ldots, i_{q} \leq m} a_{i_{1} \cdots i_{q}} \prod_{s=1}^{q} \mathbf{X}^{\left(i_{s}\right)}$ and the matrix $\mathbf{W}_{\mathbf{X}}=\mathbf{F}_{\mathbf{X}} \mathbf{F}_{\mathbf{X}}^{*}$.

Let $\mathbf{Y}^{(1)}, \ldots, \mathbf{Y}^{(q)}$ be Gaussian random matrices as in Section 1, and let $\mathbf{W}_{\mathbf{Y}}$ be defined analogously to $\mathbf{W}_{\mathbf{X}}$. Let $\mathcal{G}_{\mathbf{X}}(x)$ and $\mathcal{G}_{\mathbf{Y}}(x)$ denote the empirical spectral distribution functions of the matrices $\mathbf{W}_{\mathbf{X}}$ and $\mathbf{W}_{\mathbf{Y}}$, respectively.

Theorem 8.11. Let $\mathbf{E} X_{j k}^{(q)}=0$ and $\mathbf{E}\left|X_{j k}^{(q)}\right|^{2}=1$. Assume that the random variables $X_{j k}^{(q)}$ for $q=1, \ldots, m ; j, k=1, \ldots, m$ satisfy the Lindeberg condition (3.1). Then

$$
\lim _{n \rightarrow \infty}\left(\mathcal{G}_{\mathbf{X}}(x)-\mathcal{G}_{\mathbf{Y}}(x)\right)=0 \quad \text { in probability }
$$


Sketch of Proof. Similarly as in Sections 8.1.2 and 8.1.4 we may check the conditions (3.2) and (3.20) - 3.25) of Theorem 3.2 for each monomial functional $\mathbb{F}$. Using linearity and the boundedness of the resolvents $\mathbf{R}_{\mathbf{Z}}$, we may conclude that all these condition hold for the polynomial functional $\mathbb{F}$. Thus, by Theorem 3.2 , the matrices $\mathbf{W}_{\mathbf{X}}$ and $\mathbf{W}_{\mathbf{Y}}$ have the same limiting empirical spectral distribution, and Theorem 8.11 is proved.

Remark 8.12. There has recently been considerable progress in computing the limiting spectral distributions for polynomials of random matrices; see the approach by Belinschi, Mai and Speicher [9] for self-adjoint polynomials of self-adjoint random matrices. Possibly this approach can also be used to compute the limiting distributions in Theorem 8.11

\subsubsection{Spherical Ensemble}

In this section we consider the so-called spherical ensemble. Assume that the $X_{j k}^{(q)}, q=1,2$, $j, k=1, \ldots, n$, are independent random variables as in (8.1) or (8.2). Moreover, assume that the r.v.'s $X_{j k}^{(2)}$ satisfy the condition

$$
\max _{j, k} \mathbf{E}\left|X_{j k}^{(2)}\right|^{2} \mathbb{I}\left\{\left|X_{j k}^{(2)}\right|>M\right\} \rightarrow 0, \quad \text { as } \quad M \rightarrow \infty .
$$

Let $\mathbf{F}=\mathbf{X}^{(1)}\left(\mathbf{X}^{(2)}\right)^{-1}$, where $\mathbf{X}^{(1)}$ and $\mathbf{X}^{(2)}$ denote the $n \times n$ matrices with the entries $\frac{1}{\sqrt{n}} X_{j k}^{(1)}$ and $\frac{1}{\sqrt{n}} X_{j k}^{(2)}$, respectively.

Remark. It is well-known that under Condition (8.23), the matrix $\mathbf{X}^{(2)}$ is invertible with probability $1+o(1)$ as $n \rightarrow \infty$, see e.g. Lemma A.5 in Appendix A.3. Thus, since we are interested in convergence in probability, we may restrict ourselves to the event where $\mathbf{X}^{(2)}$ is invertible. This will tacitly be assumed in the subsequent proofs.

Let $\mathbf{W}=\mathbf{F F}^{*}$, and let $\mathcal{G}_{n}(x)$ denote the empirical spectral distribution function of the matrix $\mathbf{W}$. Then we have the following result, cf. Tikhomirov [44.

Theorem 8.13. Assume that the random variables $X_{j k}^{(q)}$, for $q=1,2$ and $j, k=1, \ldots, n$ satisfy the Lindeberg condition (3.1). Also, assume that Condition (8.23) holds. Then

$$
\lim _{n \rightarrow \infty} \mathcal{G}_{n}(x)=G(x) \quad \text { in probability }
$$

where $g(x)=G^{\prime}(x)=\frac{1}{\pi} \frac{1}{\sqrt{x}(1+x)} \mathbb{I}\{x \geq 0\}$.

Remark. Note that if $\xi$ has Cauchy density then $\eta=\xi^{2}$ has density $p(x)$.

Proof. In order to apply Theorem 3.2 we need to regularize the inverse matrix $\left(\mathbf{X}^{(2)}\right)^{-1}$. To begin with, note that $\left(\mathbf{X}^{(2)}\right)^{-1}=\left(\left(\mathbf{X}^{(2)}\right)^{*} \mathbf{X}^{(2)}\right)^{-1}\left(\mathbf{X}^{(2)}\right)^{*}=\left(\mathbf{X}^{(2)}\right)^{*}\left(\mathbf{X}^{(2)}\left(\mathbf{X}^{(2)}\right)^{*}\right)^{-1}$. We now introduce the following matrices. For any $t>0$, let

$$
\begin{aligned}
\mathbf{A}_{t}=\left(\left(\mathbf{X}^{(2)}\right)^{*} \mathbf{X}^{(2)}+t \mathbf{I}\right)^{-1}, & \widetilde{\mathbf{A}}_{t}=\left(\mathbf{X}^{(2)}\left(\mathbf{X}^{(2)}\right)^{*}+t \mathbf{I}\right)^{-1}, \\
\left(\mathbf{X}^{(2)}\right)_{t}^{-1}=\mathbf{A}_{t}\left(\mathbf{X}^{(2)}\right)^{*}=\left(\mathbf{X}^{(2)}\right)^{*} \widetilde{\mathbf{A}}_{t}, & \mathbf{F}_{t}=\mathbf{X}^{(1)}\left(\mathbf{X}^{(2)}\right)_{t}^{-1}, \quad \mathbf{W}_{t}=\mathbf{F}_{t} \mathbf{F}_{t}^{*} .
\end{aligned}
$$

Also, let $s_{t}(z)=\frac{1}{n} \operatorname{Tr} \mathbf{R}_{t}$ and $s(z)=\frac{1}{n} \operatorname{Tr} \mathbf{R}$, where $\mathbf{R}_{t}=\left(\mathbf{W}_{t}-z \mathbf{I}\right)^{-1}, \mathbf{R}=(\mathbf{W}-z \mathbf{I})^{-1}$, and $z=u+i v, v>0$. We prove the following. 
Lemma 8.14. Under condition (8.23), we have

$$
\lim _{t \rightarrow 0} \limsup _{n \rightarrow \infty}\left|s_{t}(z)-s(z)\right|=0 \quad \text { in probability. }
$$

Proof. Write

$$
\mathbf{R}_{t}-\mathbf{R}=\int_{0}^{t} \frac{d \mathbf{R}_{u}}{d u} d u=-\int_{0}^{t} \mathbf{R}_{u} \frac{d \mathbf{W}_{u}}{d u} \mathbf{R}_{u} d u=2 \int_{0}^{t} \mathbf{R}_{u} \mathbf{F}_{u} \widetilde{\mathbf{A}}_{u} \mathbf{F}_{u}^{*} \mathbf{R}_{u} d u
$$

Because the matrix $\widetilde{\mathbf{A}}_{t}$ is positive definite, we have

$$
\left|\operatorname{Tr}\left(\mathbf{R}_{u} \mathbf{F}_{u} \widetilde{\mathbf{A}}_{u} \mathbf{F}_{u}^{*} \mathbf{R}_{u}\right)\right| \leq \operatorname{Tr} \widetilde{\mathbf{A}}_{u}\left\|\mathbf{R}_{u} \mathbf{F}_{u}\right\|\left\|\mathbf{F}_{u}^{*} \mathbf{R}_{u}\right\| .
$$

Also, for any $u>0$, we have

$$
\left\|\mathbf{R}_{u} \mathbf{F}_{u}\right\|^{2} \leq v^{-1}\left(1+|z| v^{-1}\right) \quad \text { and } \quad\left\|\mathbf{F}_{u}^{*} \mathbf{R}_{u}\right\|^{2} \leq v^{-1}\left(1+|z| v^{-1}\right) .
$$

We therefore obtain

$$
\left|s_{t}(z)-s_{0}(z)\right| \leq 2 v^{-1}\left(1+|z| v^{-1}\right) \int_{0}^{t} \frac{1}{n} \operatorname{Tr} \widetilde{\mathbf{A}}_{u} d u .
$$

Let $s_{1} \geq \cdots \geq s_{n}$ denote the singular values of the matrix $\mathbf{X}^{(2)}$. Then the integral in (8.27) may be represented as

$$
\int_{0}^{t} \frac{1}{n} \operatorname{Tr} \widetilde{\mathbf{A}}_{u} d u=\frac{1}{n} \sum_{k=1}^{n} \int_{0}^{t}\left(s_{k}^{2}+u\right)^{-1} d u=\frac{1}{n} \sum_{k=1}^{n}\left(\log \left(s_{k}^{2}+t\right)-\log \left(s_{k}^{2}\right)\right) .
$$

Now, by the Marchenko-Pastur theorem (Theorem 8.1), we have, for any fixed $t>0$,

$$
\lim _{n \rightarrow \infty} \frac{1}{n} \sum_{k=1}^{n} \log \left(s_{k}^{2}+t\right)=\int_{0}^{4} \log (x+t) \frac{1}{2 \pi} \sqrt{(4-x) / x} d x \quad \text { in probability } .
$$

By Assumption (8.23) and Lemmas A.4 - A.6 in Appendix A.3, the matrix $\mathbf{X}^{(2)}$ satisfies Conditions $(C 0),(C 1)$ and $(C 2)$. Thus, by the Marchenko-Pastur theorem and Lemma A.9, we also have

$$
\lim _{n \rightarrow \infty} \frac{1}{n} \sum_{k=1}^{n} \log \left(s_{k}^{2}\right)=\int_{0}^{4} \log (x) \frac{1}{2 \pi} \sqrt{(4-x) / x} d x \quad \text { in probability . }
$$

Now fix $\varepsilon>0$, and take $t>0$ sufficiently small so that

$$
\int_{0}^{4}(\log (x+t)-\log (x)) \frac{1}{2 \pi} \sqrt{(4-x) / x} d x \leq \frac{\varepsilon}{3}
$$


It then follows from (8.28) - (8.30) that

$$
\begin{aligned}
\lim _{n \rightarrow \infty} \operatorname{Pr} & \left\{\int_{0}^{t} \frac{1}{n} \operatorname{Tr} \widetilde{\mathbf{A}}_{u} d u \geq \varepsilon\right\} \\
\leq \lim _{n \rightarrow \infty} \operatorname{Pr} & \left\{\left|\frac{1}{n} \sum_{k=1}^{n} \log \left(s_{k}^{2}+t\right)-\int_{0}^{4} \log (x+t) \frac{1}{2 \pi} \sqrt{(4-x) / x} d x\right| \geq \frac{\varepsilon}{3}\right\} \\
& +\lim _{n \rightarrow \infty} \operatorname{Pr}\left\{\left|\frac{1}{n} \sum_{k=1}^{n} \log \left(s_{k}^{2}\right)-\int_{0}^{4} \log (x) \frac{1}{2 \pi} \sqrt{(4-x) / x} d x\right| \geq \frac{\varepsilon}{3}\right\}=0 .
\end{aligned}
$$

In view of (8.27), this implies the statement of the lemma.

Now it is enough to determine the limit distribution of the singular values of the matrix $\mathbf{F}_{t}$ for fixed $t>0$ and then to take the limit as $t \rightarrow 0$ to find the limit distribution of the singular values of the matrix $\mathbf{F}$. We check that the conditions of Theorem 3.2 hold for the matrix-valued function $\mathbb{F}_{t}\left(\mathbf{X}^{(1)}, \mathbf{X}^{(2)}\right)=\mathbf{X}^{(1)}\left(\mathbf{X}^{(2)}\right)_{t}^{-1}$. Let $\mathbf{F}_{t}(\mathbf{X})=\mathbf{X}^{(1)}\left(\mathbf{X}^{(2)}\right)_{t}^{-1}$ and $\mathbf{F}_{t}(\mathbf{Y})=\mathbf{Y}^{(1)}\left(\mathbf{Y}^{(2)}\right)_{t}^{-1}$, where the $\mathbf{Y}^{(q)}$ denote random matrices with independent Gaussian entries as in Section 1. Also, let $\mathbf{A}_{t}\left(\mathbf{X}^{(2)}\right)=\left(\left(\mathbf{X}^{(2)}\right)^{*} \mathbf{X}^{(2)}+t \mathbf{I}\right)^{-1}$ and $\mathbf{A}_{t}\left(\mathbf{Y}^{(2)}\right)=$ $\left(\left(\mathbf{Y}^{(2)}\right)^{*} \mathbf{Y}^{(2)}+t \mathbf{I}\right)^{-1}$. We first check the rank condition (3.2). Clearly,

$$
\begin{aligned}
\operatorname{rank} & \left\{\mathbf{F}_{t}(\mathbf{X})-\mathbf{F}_{t}(\mathbf{Y})\right\} \\
& \leq \operatorname{rank}\left(\mathbf{X}^{(1)}-\mathbf{Y}^{(1)}\right)+\operatorname{rank}\left(\mathbf{A}_{t}\left(\mathbf{X}^{(2)}\right)-\mathbf{A}_{t}\left(\mathbf{Y}^{(2)}\right)\right)+\operatorname{rank}\left(\mathbf{X}^{(2)}-\mathbf{Y}^{(2)}\right)^{*} \\
& \leq \operatorname{rank}\left(\mathbf{X}^{(1)}-\mathbf{Y}^{(1)}\right)+3 \operatorname{rank}\left(\mathbf{X}^{(2)}-\mathbf{Y}^{(2)}\right) .
\end{aligned}
$$

Thus, the rank condition (3.2) holds with $C\left(\mathbb{F}_{t}\right)=3$. We now check conditions (3.20), (3.22) and (3.24). As usual, we restrict ourselves to the real case for simplicity. By (3.19), we have

$$
g_{j k}^{(q)}=\operatorname{Tr} \frac{\partial \mathbf{V}}{\partial Z_{j k}^{(q)}} \mathbf{R}^{2}
$$

where

$$
\mathbf{V}=\left[\begin{array}{cc}
\mathbf{O} & \mathbf{F}_{t}(\mathbf{Z}) \\
\mathbf{F}_{t}^{*}(\mathbf{Z}) & \mathbf{O}
\end{array}\right] \text { and } \quad \mathbf{R}:=(\mathbf{V}-z \mathbf{I})^{-1}
$$

Introduce the matrices

$$
\mathbf{H}^{(1)}=\left[\begin{array}{cc}
\mathbf{Z}^{(1)} & \mathbf{O} \\
\mathbf{O} & \mathbf{Z}^{(2)} \mathbf{A}_{t}
\end{array}\right], \quad \mathbf{H}^{(2)}=\left[\begin{array}{cc}
\mathbf{A}_{t}\left(\mathbf{Z}^{(2)}\right)^{*} & \mathbf{O} \\
\mathbf{O} & \left(\mathbf{Z}^{(1)}\right)^{*}
\end{array}\right],
$$

where now $\mathbf{A}_{t}=\left(\left(\mathbf{Z}^{(2)}\right)^{*} \mathbf{Z}^{(2)}+t \mathbf{I}\right)^{-1}$. We have the representation

$$
\mathbf{V}=\mathbf{H}^{(1)} \mathbf{H}^{(2)} \mathbf{J}
$$

where $\mathbf{J}=\mathbf{J}(-1)$. (Recall that $\mathbf{J}(\alpha)$ was defined in (5.17).) Denote by $\mathbf{e}_{j}, j=1, \ldots, 2 n$, the vectors of the standard orthonormal basis of $\mathbb{R}^{2 n}$. First we note, for $q=1$ and 
$j, k=1, \ldots, n$,

$$
\frac{\partial \mathbf{V}}{\partial Z_{j k}^{(1)}}=\mathbf{e}_{j} \mathbf{e}_{k}^{T} \mathbf{H}^{(2)} \mathbf{J}+\mathbf{H}^{(1)} \mathbf{e}_{k+n} \mathbf{e}_{j+n}^{T} \mathbf{J} .
$$

Applying Hölder's inequality, we get

$$
\left|g_{j k}^{(1)}(\theta)\right| \leq\left\|\mathbf{e}_{k}^{T} \mathbf{H}^{(2)} \mathbf{J}\right\|_{2}\left\|\mathbf{R}^{2} \mathbf{e}_{j}\right\|_{2}+\left\|\mathbf{e}_{j+n}^{T} \mathbf{J R}^{2}\right\|_{2}\left\|\mathbf{H}^{(1)} \mathbf{e}_{k+n}\right\|_{2} .
$$

This implies that

$$
\begin{aligned}
& \mathbf{E}\left\{\left|g_{j k}^{(1)}(\theta)\right| \mid X_{j k}^{(1)}, Y_{j k}^{(1)}\right\} \\
& \quad \leq v^{-2} \mathbf{E}\left\{\left\|\mathbf{e}_{k}^{T} \mathbf{H}^{(2)} \mathbf{J}\right\|_{2} \mid X_{j k}^{(1)}, Y_{j k}^{(1)}\right\}+v^{-2} \mathbf{E}\left\{\left\|\mathbf{H}^{(1)} \mathbf{e}_{k+n}\right\|_{2} \mid X_{j k}^{(1)}, Y_{j k}^{(1)}\right\} .
\end{aligned}
$$

Note that

$$
\left\|\mathbf{e}_{k}^{T} \mathbf{H}^{(2)} \mathbf{J}\right\|_{2}=\left\|\overline{\mathbf{e}}_{k}^{T} \mathbf{A}_{t}\left(\mathbf{Z}^{(2)}\right)^{*}\right\|_{2}, \quad\left\|\mathbf{H}^{(1)} \mathbf{e}_{k+n}\right\|_{2}=\left\|\mathbf{Z}^{(2)} \mathbf{A}_{t} \overline{\mathbf{e}}_{k}\right\|_{2},
$$

where $\overline{\mathbf{e}}_{k}$ denotes the corresponding standard basis vector of $\mathbb{R}^{n}$. Because the matrices $\mathbf{Z}^{(2)}$ and the r.v.'s $Z_{j k}^{(1)}$ are independent and $\left\|\mathbf{Z}^{(2)} \mathbf{A}_{t}\right\| \leq t^{-\frac{1}{2}}$, we get

$$
\mathbf{E}\left\{\left|g_{j k}^{(1)}(\theta)\right| \mid X_{j k}^{(1)}, Y_{j k}^{(1)}\right\} \leq C v^{-2} t^{-\frac{1}{2}}
$$

Consider the function $g_{j k}^{(2)}$ now. Introduce some auxiliary matrices. Let

$$
\mathbf{L}_{t}=\left[\begin{array}{cc}
\mathbf{A}_{t} & \mathbf{O} \\
\mathbf{O} & \mathbf{A}_{t}
\end{array}\right], \quad \mathbf{M}_{t}=\left[\begin{array}{cc}
\mathbf{Z}^{(2)} & \mathbf{O} \\
\mathbf{O} & \mathbf{Z}^{(2)}
\end{array}\right]
$$

With this notation, we have

$$
\frac{\partial \mathbf{H}^{(1)}}{\partial Z_{j k}^{(2)}}=\mathbf{e}_{j+n} \mathbf{e}_{k+n}^{T} \mathbf{L}_{t}-\mathbf{M}_{t} \mathbf{L}_{t} \mathbf{e}_{k+n} \mathbf{e}_{j+n}^{T} \mathbf{M}_{t} \mathbf{L}_{t}-\mathbf{M}_{t} \mathbf{L}_{t} \mathbf{M}_{t}^{*} \mathbf{e}_{j+n} \mathbf{e}_{k+n}^{T} \mathbf{L}_{t}
$$

and

$$
\frac{\partial \mathbf{H}^{(2)}}{\partial Z_{j k}^{(2)}}=\mathbf{L}_{t} \mathbf{e}_{k} \mathbf{e}_{j}^{T}-\mathbf{L}_{t} \mathbf{e}_{k} \mathbf{e}_{j}^{T} \mathbf{M}_{t} \mathbf{L}_{t} \mathbf{M}_{t}^{*}-\mathbf{L}_{t} \mathbf{M}_{t}^{*} \mathbf{e}_{j} \mathbf{e}_{k}^{T} \mathbf{L}_{t} \mathbf{M}_{t}^{*}
$$

Furthermore,

$$
\frac{\partial \mathbf{V}}{\partial Z_{j k}^{(2)}}=\frac{\partial \mathbf{H}^{(1)}}{\partial Z_{j k}^{(2)}} \mathbf{H}^{(2)} \mathbf{J}+\mathbf{H}^{(1)} \frac{\partial \mathbf{H}^{(2)}}{\partial Z_{j k}^{(2)}} \mathbf{J} .
$$


By Hölder's inequality, we have

$$
\begin{aligned}
\left|g_{j k}^{(2)}\right| & \leq\left\|\mathbf{e}_{k+n}^{T} \mathbf{L}_{t} \mathbf{H}^{(2)} \mathbf{J}\right\|_{2}\left\|\mathbf{R}^{2} \mathbf{e}_{j+n}\right\|_{2} \\
& +\left\|\mathbf{e}_{j+n}^{T} \mathbf{M}_{t} \mathbf{L}_{t} \mathbf{H}^{(2)} \mathbf{J}\right\|_{2}\left\|\mathbf{R}^{2} \mathbf{M}_{t} \mathbf{L}_{t} \mathbf{e}_{k+n}\right\|_{2}+\left\|\mathbf{e}_{k+n}^{T} \mathbf{L}_{t} \mathbf{H}^{(2)} \mathbf{J}\right\|_{2}\left\|\mathbf{R}^{2} \mathbf{M}_{t} \mathbf{L}_{t} \mathbf{M}_{t}^{*} \mathbf{e}_{j+n}\right\|_{2} \\
& +\left\|\mathbf{e}_{j}^{T} \mathbf{J}\right\|_{2}\left\|\mathbf{R}^{2} \mathbf{H}^{(1)} \mathbf{L}_{t} \mathbf{e}_{k}\right\|_{2} \\
& +\left\|\mathbf{e}_{j}^{T} \mathbf{M}_{t} \mathbf{L}_{t} \mathbf{M}_{t}^{*} \mathbf{J}\right\|_{2}\left\|\mathbf{R}^{2} \mathbf{H}^{(1)} \mathbf{L}_{t} \mathbf{e}_{k}\right\|_{2}+\left\|\mathbf{e}_{k}^{T} \mathbf{L}_{t} \mathbf{M}_{t}^{*} \mathbf{J}\right\|_{2}\left\|\mathbf{R}^{2} \mathbf{H}^{(1)} \mathbf{L}_{t} \mathbf{M}_{t}^{*} \mathbf{e}_{j}\right\|_{2} .
\end{aligned}
$$

Simple calculations show that

$$
\begin{aligned}
\left|g_{j k}^{(2)}\right| & \leq v^{-2}\left\|\overline{\mathbf{e}}_{k}^{T} \mathbf{A}_{t}\left(\mathbf{Z}^{(1)}\right)^{*}\right\|_{2}\left\|\overline{\mathbf{e}}_{j}\right\|_{2} \\
& +v^{-2}\left\|\overline{\mathbf{e}}_{j}^{T} \mathbf{Z}^{(2)} \mathbf{A}_{t}\left(\mathbf{Z}^{(1)}\right)^{*}\right\|_{2}\left\|\mathbf{Z}^{(2)} \mathbf{A}_{t} \overline{\mathbf{e}}_{k}\right\|_{2}+v^{-2}\left\|\overline{\mathbf{e}}_{k}^{T} \mathbf{A}_{t}\left(\mathbf{Z}^{(1)}\right)^{*}\right\|_{2}\left\|\mathbf{Z}^{(2)} \mathbf{A}_{t}\left(\mathbf{Z}^{(2)}\right)^{*} \overline{\mathbf{e}}_{j}\right\|_{2} \\
& +v^{-2}\left\|\overline{\mathbf{e}}_{j}^{T}\right\|_{2}\left\|\mathbf{Z}^{(1)} \mathbf{A}_{t} \overline{\mathbf{e}}_{k}\right\|_{2} \\
& +v^{-2}\left\|\overline{\mathbf{e}}_{j}^{T} \mathbf{Z}^{(2)} \mathbf{A}_{t}\left(\mathbf{Z}^{(2)}\right)^{*}\right\|_{2}\left\|\mathbf{Z}^{(1)} \mathbf{A}_{t} \overline{\mathbf{e}}_{k}\right\|_{2}+v^{-2}\left\|\overline{\mathbf{e}}_{k}^{T} \mathbf{A}_{t}\left(\mathbf{Z}^{(2)}\right)^{*}\right\|_{2}\left\|\mathbf{Z}^{(1)} \mathbf{A}_{t}\left(\mathbf{Z}^{(2)}\right)^{*} \overline{\mathbf{e}}_{j}\right\|_{2} .
\end{aligned}
$$

By definition of $\mathbf{A}_{t}$, we have

$$
\left\|\mathbf{A}_{t}\right\| \leq t^{-1}, \quad\left\|\mathbf{Z}^{(2)} \mathbf{A}_{t}\right\| \leq t^{-1 / 2}, \quad\left\|\mathbf{Z}^{(2)} \mathbf{A}_{t}\left(\mathbf{Z}^{(2)}\right)^{*}\right\| \leq 1
$$

Combining the last two relations, we get

$$
\begin{aligned}
\mathbf{E}\left\{\left|g_{j k}^{(2)}(\theta)\right| \mid X_{j k}^{(2)}, Y_{j k}^{(2)}\right\} & \leq v^{-2} \mathbf{E}\left\{\left\|\overline{\mathbf{e}}_{k}^{T} \mathbf{A}_{t}\left(\mathbf{Z}^{(1)}\right)^{*}\right\|_{2} \mid X_{j k}^{(2)}, Y_{j k}^{(2)}\right\} \\
& +v^{-2} t^{-1 / 2} \mathbf{E}\left\{\left\|\overline{\mathbf{e}}_{j}^{T} \mathbf{Z}^{(2)} \mathbf{A}_{t}\left(\mathbf{Z}^{(1)}\right)^{*}\right\|_{2} \mid X_{j k}^{(2)}, Y_{j k}^{(2)}\right\} \\
& +v^{-2} \mathbf{E}\left\{\left\|\overline{\mathbf{e}}_{k}^{T} \mathbf{A}_{t}\left(\mathbf{Z}^{(1)}\right)^{*}\right\|_{2} \mid X_{j k}^{(2)}, Y_{j k}^{(2)}\right\} \\
& +v^{-2} \mathbf{E}\left\{\left\|\mathbf{Z}^{(1)} \mathbf{A}_{t} \overline{\mathbf{e}}_{k}\right\|_{2} \mid X_{j k}^{(2)}, Y_{j k}^{(2)}\right\} \\
& +v^{-2} \mathbf{E}\left\{\left\|\mathbf{Z}^{(1)} \mathbf{A}_{t} \overline{\mathbf{e}}_{k}\right\|_{2} \mid X_{j k}^{(2)}, Y_{j k}^{(2)}\right\} \\
& +v^{-2} t^{-1 / 2} \mathbf{E}\left\{\left\|\mathbf{Z}^{(1)} \mathbf{A}_{t}\left(\mathbf{Z}^{(2)}\right)^{*} \overline{\mathbf{e}}_{j}\right\|_{2} \mid X_{j k}^{(2)}, Y_{j k}^{(2)}\right\} .
\end{aligned}
$$

Now, writing $\mathbf{Z}^{(1)}=\left(\mathbf{Z}^{(1)}-\mathbf{E} \mathbf{Z}^{(1)}\right)+\mathbf{E} \mathbf{Z}^{(1)}$ and using Eqs. (3.3) and (3.9), it is straightforward to check that, for any constant vector $\mathbf{v}$, we have $\mathbf{E}\left\|\mathbf{Z}^{(1)} \mathbf{v}\right\|_{2}^{2} \leq C\|\mathbf{v}\|_{2}^{2}$. Thus, because the matrix $\mathbf{Z}^{(1)}$ and the r.v.'s $X_{j k}^{(2)}, Y_{j k}^{(2)}$ are independent, we obtain

$$
\begin{array}{r}
\mathbf{E}\left\{\left\|\overline{\mathbf{e}}_{j}^{T} \mathbf{Z}^{(2)} \mathbf{A}_{t} \mathbf{Z}^{(1)^{*}}\right\|_{2}^{2} \mid X_{j k}^{(2)}, Y_{j k}^{(2)}\right\} \\
\quad \leq C t^{-1}, \\
\mathbf{E}\left\{\left\|\overline{\mathbf{e}}_{j}^{T} \mathbf{A}_{t} \mathbf{Z}^{(1)^{*}}\right\|_{2}^{2} \mid X_{j k}^{(2)}, Y_{j k}^{(2)}\right\} \leq C t^{-2} .
\end{array}
$$

Inserting the bounds (8.36) and (8.37) into (8.35), we get

$$
\mathbf{E}\left\{\left|g_{j k}^{(2)}(\theta)\right| \mid X_{j k}^{(2)}, Y_{j k}^{(2)}\right\} \leq C v^{-2}\left(t^{-2}+t^{-1}\right) .
$$


This inequality implies condition (3.20) for $q=2$. Now we consider the condition (3.22). We have

$$
\frac{\partial g_{j k}^{(q)}}{\partial Z_{j k}^{(q)}}=\operatorname{Tr} \frac{\partial^{2} \mathbf{V}}{\partial\left(Z_{j k}^{(q)}\right)^{2}} \mathbf{R}^{2}-\operatorname{Tr} \frac{\partial \mathbf{V}}{\partial Z_{j k}^{(q)}} \mathbf{R} \frac{\partial \mathbf{V}}{\partial Z_{j k}^{(q)}} \mathbf{R}^{2}-\operatorname{Tr} \frac{\partial \mathbf{V}}{\partial Z_{j k}^{(q)}} \mathbf{R}^{2} \frac{\partial \mathbf{V}}{\partial Z_{j k}^{(q)}} \mathbf{R} .
$$

Using representation (8.31), it is straightforward to check that

$$
\frac{\partial^{2} \mathbf{V}}{\partial\left(Z_{j k}^{(1)}\right)^{2}}=0
$$

This equality and (8.38) together imply

$$
\begin{aligned}
\frac{\partial g_{j k}^{(1)}}{\partial Z_{j k}^{(1)}} & =-2 \operatorname{Tr}\left(\frac{\partial \mathbf{V}}{\partial Z_{j k}^{(1)}} \mathbf{R}\right)^{2} \mathbf{R} \\
& =-2 \operatorname{Tr}\left(\mathbf{e}_{j} \mathbf{e}_{k}^{T} \mathbf{H}^{(2)} \mathbf{J R}+\mathbf{H}^{(1)} \mathbf{e}_{k+n} \mathbf{e}_{j+n}^{T} \mathbf{J R}\right)^{2} \mathbf{R}=-2\left(T_{1}+T_{2}+T_{3}+T_{4}\right),
\end{aligned}
$$

where

$$
\begin{aligned}
& T_{1}=\operatorname{Tr} \mathbf{e}_{j} \mathbf{e}_{k}^{T} \mathbf{H}^{(2)} \mathbf{J} \mathbf{R e}_{j} \mathbf{e}_{k}^{T} \mathbf{H}^{(2)} \mathbf{J R}^{2}, \\
& T_{2}=\operatorname{Tr} \mathbf{e}_{j} \mathbf{e}_{k}^{T} \mathbf{H}^{(2)} \mathbf{J R} \mathbf{H}^{(1)} \mathbf{e}_{k+n} \mathbf{e}_{j+n}^{T} \mathbf{J} \mathbf{R}^{2}, \\
& T_{3}=\operatorname{Tr} \mathbf{H}^{(1)} \mathbf{e}_{k+n} \mathbf{e}_{j+n}^{T} \mathbf{J R} \mathbf{e}_{j} \mathbf{e}_{k}^{T} \mathbf{H}^{(2)} \mathbf{J R}^{2}, \\
& T_{4}=\operatorname{Tr} \mathbf{H}^{(1)} \mathbf{e}_{k+n} \mathbf{e}_{j+n}^{T} \mathbf{J R} \mathbf{H}^{(1)} \mathbf{e}_{k+n} \mathbf{e}_{j+n}^{T} \mathbf{J} \mathbf{R}^{2} .
\end{aligned}
$$

It is easy to see that

$$
\begin{aligned}
& \left|T_{1}\right| \leq\left|\mathbf{e}_{k}^{T} \mathbf{H}^{(2)} \mathbf{J R e}_{j}\left\|\mathbf{e}_{k}^{T} \mathbf{H}^{(2)} \mathbf{J R}^{2} \mathbf{e}_{j} \mid \leq v^{-3}\right\| \mathbf{e}_{k}^{T} \mathbf{H}^{(2)} \|_{2}^{2} \leq v^{-3} t^{-1},\right. \\
& \left|T_{2}\right| \leq\left|\mathbf{e}_{k}^{T} \mathbf{H}^{(2)} \mathbf{J R} \mathbf{H}^{(1)} \mathbf{e}_{k+n}\left\|\mathbf{e}_{j+n}^{T} \mathbf{J R}^{2} \mathbf{e}_{j} \mid \leq v^{-3}\right\| \mathbf{e}_{k}^{T} \mathbf{H}^{(2)}\left\|_{2}\right\| \mathbf{H}^{(1)} \mathbf{e}_{k+n} \|_{2} \leq v^{-3} t^{-1},\right. \\
& \left|T_{3}\right| \leq\left|\mathbf{e}_{j+n}^{T} \mathbf{J R e}_{j}\left\|\mathbf{e}_{k}^{T} \mathbf{H}^{(2)} \mathbf{J R}^{2} \mathbf{H}^{(1)} \mathbf{e}_{k+n} \mid \leq v^{-3}\right\| \mathbf{H}^{(1)} \mathbf{e}_{k+n}\left\|_{2}\right\| \mathbf{e}_{k}^{T} \mathbf{H}^{(2)} \|_{2} \leq v^{-3} t^{-1},\right. \\
& \left|T_{4}\right| \leq\left|\mathbf{e}_{j+n}^{T} \mathbf{J R H}^{(1)} \mathbf{e}_{k+n}\right|\left|\mathbf{e}_{j+n}^{T} \mathbf{J R}^{2} \mathbf{H}^{(1)} \mathbf{e}_{k+n}\right| \leq v^{-3}\left\|\mathbf{H}^{(1)} \mathbf{e}_{k+n}\right\|_{2}^{2} \leq v^{-3} t^{-1} .
\end{aligned}
$$

This implies that

$$
\mathbf{E}\left\{\left|\frac{\partial g_{j k}^{(1)}}{\partial Z_{j k}^{(1)}}\right| \mid X_{j k}^{(1)}, Y_{j k}^{(1)}\right\} \leq C v^{-3} t^{-1} .
$$

Thus condition (3.22) holds for $q=1$. Consider $q=2$ now. We have

$$
\frac{\partial g_{j k}^{(2)}}{\partial Z_{j k}^{(2)}}=-2 \operatorname{Tr}\left(\frac{\partial \mathbf{V}}{\partial Z_{j k}^{(2)}} \mathbf{R}\right)^{2} \mathbf{R}+\operatorname{Tr} \frac{\partial^{2} \mathbf{V}}{\partial\left(Z_{j k}^{(2)}\right)^{2}} \mathbf{R}^{2}
$$


Using formula (8.34), we get

$$
\frac{\partial^{2} \mathbf{V}}{\partial\left(Z_{j k}^{(2)}\right)^{2}}=\frac{\partial^{2} \mathbf{H}^{(1)}}{\partial\left(Z_{j k}^{(2)}\right)^{2}} \mathbf{H}^{(2)} \mathbf{J}+2 \frac{\partial \mathbf{H}^{(1)}}{\partial Z_{j k}^{(2)}} \frac{\partial \mathbf{H}^{(2)}}{\partial Z_{j k}^{(2)}} \mathbf{J}+\mathbf{H}^{(1)} \frac{\partial^{2} \mathbf{H}^{(2)}}{\partial\left(Z_{j k}^{(2)}\right)^{2}} \mathbf{J} .
$$

Introduce the matrices

$$
\begin{aligned}
& \mathbf{P}_{t}^{(1)}=\mathbf{e}_{k+n} \mathbf{e}_{j+n}^{T} \mathbf{M}_{t}+\mathbf{M}_{t}^{*} \mathbf{e}_{j+n} \mathbf{e}_{k+n}^{T}, \\
& \mathbf{P}_{t}^{(2)}=\mathbf{e}_{k} \mathbf{e}_{j}^{T} \mathbf{M}_{t}+\mathbf{M}_{t}^{*} \mathbf{e}_{j} \mathbf{e}_{k}^{T} .
\end{aligned}
$$

Simple calculations show that

$$
\frac{\partial^{2} \mathbf{H}^{(1)}}{\partial\left(Z_{j k}^{(2)}\right)^{2}}= \pm A_{1} \pm \cdots \pm A_{9}
$$

where

$$
\begin{array}{rlrl}
A_{1} & =\mathbf{e}_{j+n} \mathbf{e}_{k+n}^{T} \mathbf{L}_{t} \mathbf{P}_{t}^{(1)} \mathbf{L}_{t}, & A_{2}=\mathbf{e}_{j+n} \mathbf{e}_{k+n}^{T} \mathbf{L}_{t} \mathbf{e}_{k+n} \mathbf{e}_{j+n}^{T} \mathbf{M}_{t} \mathbf{L}_{t}, \\
A_{3}=\mathbf{M}_{t} \mathbf{L}_{t} \mathbf{P}_{t}^{(1)} \mathbf{L}_{t} \mathbf{e}_{k+n} \mathbf{e}_{j+n}^{T} \mathbf{M}_{t} \mathbf{L}_{t}, & A_{4}=\mathbf{M}_{t} \mathbf{L}_{t} \mathbf{e}_{k+n} \mathbf{e}_{j+n}^{T} \mathbf{e}_{j+n} \mathbf{e}_{k+n}^{T} \mathbf{L}_{t}, \\
A_{5}=\mathbf{M}_{t} \mathbf{L}_{t} \mathbf{e}_{k+n} \mathbf{e}_{j+n}^{T} \mathbf{M}_{t} \mathbf{L}_{t} \mathbf{P}_{t}^{(1)} \mathbf{L}_{t}, & A_{6}=\mathbf{e}_{j+n} \mathbf{e}_{k+n}^{T} \mathbf{L}_{t} \mathbf{M}_{t}^{*} \mathbf{e}_{j+n} \mathbf{e}_{k+n}^{T} \mathbf{L}_{t}, \\
A_{7}=\mathbf{M}_{t} \mathbf{L}_{t} \mathbf{P}_{t}^{(1)} \mathbf{L}_{t} \mathbf{M}_{t}^{*} \mathbf{e}_{j+n} \mathbf{e}_{k+n}^{T} \mathbf{L}_{t}, & A_{8}=\mathbf{M}_{t} \mathbf{L}_{t} \mathbf{e}_{k+n} \mathbf{e}_{j+n}^{T} \mathbf{e}_{j+n} \mathbf{e}_{k+n}^{T} \mathbf{L}_{t}, \\
A_{9}=\mathbf{M}_{t} \mathbf{L}_{t} \mathbf{M}_{t}^{*} \mathbf{e}_{j+n} \mathbf{e}_{k+n}^{T} \mathbf{L}_{t} \mathbf{P}_{t}^{(1)} \mathbf{L}_{t} . &
\end{array}
$$

Using Hölder's inequality, we may prove that

$$
\begin{array}{r}
\left|\operatorname{Tr} A_{i} \mathbf{H}^{(2)} \mathbf{J} \mathbf{R}^{2}\right| \leq C v^{-2}\left(t^{-1}+t^{-\frac{1}{2}}\right)\left(\left\|\overline{\mathbf{e}}_{j}^{T} \mathbf{Z}^{(2)} \mathbf{A}_{t} \mathbf{Z}^{(1)^{*}}\right\|_{2}+\left\|\overline{\mathbf{e}}_{j}^{T} \mathbf{A}_{t} \mathbf{Z}^{(1)^{*}}\right\|_{2}\right), \\
\text { for } i=1, \ldots, 9 .
\end{array}
$$

Using inequality (8.36) and (8.37), we therefore obtain

$$
\mathbf{E}\left\{\left|\operatorname{Tr} \frac{\partial^{2} \mathbf{H}^{(1)}}{\left(\partial Z_{j k}^{(2)}\right)^{2}} \mathbf{H}^{(2)} \mathbf{J R}^{2}\right| \mid X_{j k}^{(2)}, Y_{j k}^{(2)}\right\} \leq C v^{-2}\left(t^{-2}+t^{-1}\right) .
$$

Analogously we get

$$
\frac{\partial^{2} \mathbf{H}^{(2)}}{\partial\left(Z_{j k}^{(2)}\right)^{2}}= \pm B_{1} \pm \cdots \pm B_{9}
$$

where

$$
\begin{array}{ll}
B_{1}=\mathbf{L}_{t} \mathbf{P}_{t}^{(2)} \mathbf{L}_{t} \mathbf{e}_{k} \mathbf{e}_{j}^{T}, & B_{2}=\mathbf{L}_{t} \mathbf{P}_{t}^{(2)} \mathbf{L}_{t} \mathbf{e}_{k} \mathbf{e}_{j}^{T} \mathbf{M}_{t} \mathbf{L}_{t} \mathbf{M}_{t}^{*}, \\
B_{3}=\mathbf{L}_{t} \mathbf{e}_{k} \mathbf{e}_{j}^{T} \mathbf{e}_{j} \mathbf{e}_{k}^{T} \mathbf{L}_{t} \mathbf{M}_{t}^{*}, & B_{4}=\mathbf{L}_{t} \mathbf{e}_{k} \mathbf{e}_{j}^{T} \mathbf{M}_{t} \mathbf{L}_{t} \mathbf{P}_{t}^{(2)} \mathbf{L}_{t} \mathbf{M}_{t}^{*}, \\
B_{5}=\mathbf{L}_{t} \mathbf{e}_{k} \mathbf{e}_{j}^{T} \mathbf{M}_{t} \mathbf{L}_{t} \mathbf{e}_{k} \mathbf{e}_{j}^{T}, & B_{6}=\mathbf{L}_{t} \mathbf{P}_{t}^{(2)} \mathbf{L}_{t} \mathbf{M}_{t}^{*} \mathbf{e}_{j} \mathbf{e}_{k}^{T} \mathbf{L}_{t} \mathbf{M}_{t}^{*}, \\
B_{7}=\mathbf{L}_{t} \mathbf{e}_{k} \mathbf{e}_{j}^{T} \mathbf{e}_{j} \mathbf{e}_{k}^{T} \mathbf{L}_{t} \mathbf{M}_{t}^{*}, & B_{8}=\mathbf{L}_{t} \mathbf{M}_{t}^{*} \mathbf{e}_{j} \mathbf{e}_{k}^{T} \mathbf{L}_{t} \mathbf{P}_{t}^{(2)} \mathbf{L}_{t} \mathbf{M}_{t}^{*}, \\
B_{9}=\mathbf{L}_{t} \mathbf{M}_{t}^{*} \mathbf{e}_{j} \mathbf{e}_{k}^{T} \mathbf{L}_{t} \mathbf{e}_{k} \mathbf{e}_{j}^{T} . &
\end{array}
$$


Using Hölder's inequality we get

$$
\begin{array}{r}
\left|\operatorname{Tr} \mathbf{H}^{(1)} B_{i} \mathbf{J R}^{2}\right| \leq C v^{-2}\left(t^{-1}+t^{-\frac{1}{2}}\right)\left(\left\|\overline{\mathbf{e}}_{j} \mathbf{Z}^{(2)} \mathbf{A}_{t} \mathbf{Z}^{(1)^{*}}\right\|_{2}+\left\|\overline{\mathbf{e}}_{j} \mathbf{A}_{t} \mathbf{Z}^{(1)^{*}}\right\|_{2}\right), \\
\text { for } i=1, \ldots, 9 .
\end{array}
$$

Similarly to (8.43) we obtain

$$
\mathbf{E}\left\{\left|\operatorname{Tr} \mathbf{H}^{(1)} \frac{\partial^{2} \mathbf{H}^{(2)}}{\left(\partial Z_{j k}^{(2)}\right)^{2}} \mathbf{J R}^{2}\right| \mid X_{j k}^{(2)}, Y_{j k}^{(2)}\right\} \leq C v^{-2}\left(t^{-2}+t^{-1}\right) .
$$

Finally, using the block structure of the matrices $\mathbf{H}^{(1)}$ and $\mathbf{H}^{(2)}$, it is easy to see that

$$
\operatorname{Tr} \frac{\partial \mathbf{H}^{(1)}}{\partial Z_{j k}^{(2)}} \frac{\partial \mathbf{H}^{(2)}}{\partial Z_{j k}^{(2)}} \mathbf{J R}^{2}=0
$$

Relations (8.41), (8.43), (8.45) and (8.46) together imply

$$
\mathbf{E}\left\{\left|\operatorname{Tr} \frac{\partial^{2} \mathbf{V}}{\partial Z_{j k}^{(2)^{2}}} \mathbf{R}^{2}\right| \mid X_{j k}^{(2)}, Y_{j k}^{(2)}\right\} \leq C v^{-2}\left(t^{-2}+t^{-1}\right) .
$$

Furthermore, relations (8.32), 8.33), 8.34), together imply

$$
\left|\operatorname{Tr}\left(\frac{\partial \mathbf{V}}{\partial Z_{j k}^{(2)}} \mathbf{R}\right)^{2} \mathbf{R}\right| \leq C v^{-3}\left(t^{-1}+1\right)\left(\left\|\overline{\mathbf{e}}_{j}^{T} \mathbf{Z}^{(2)} \mathbf{A}_{t} \mathbf{Z}^{(1)^{*}}\right\|_{2}^{2}+\left\|\overline{\mathbf{e}}_{j}^{T} \mathbf{A}_{t} \mathbf{Z}^{(1)^{*}}\right\|_{2}^{2}\right)
$$

and therefore, by (8.36) and (8.37),

$$
\mathbf{E}\left\{\left|\operatorname{Tr}\left(\frac{\partial \mathbf{V}}{\partial Z_{j k}^{(2)}} \mathbf{R}\right)^{2} \mathbf{R}\right| \mid X_{j k}^{(2)}, Y_{j k}^{(2)}\right\} \leq C v^{-3}\left(t^{-3}+t^{-1}\right) .
$$

This concludes the proof of (3.22) for $q=2$. The proof of condition (3.24) is similar and hence omitted.

We may now apply Theorem 3.2 It follows from this theorem that for each $z \in \mathbb{C}$, the Stieltjes transforms $s_{t}(z ; \mathbf{X})$ and $s_{t}(z ; \mathbf{Y})$ associated with the matrices $\mathbf{W}_{t}(\mathbf{X})$ and $\mathbf{W}_{t}(\mathbf{Y})$ have the same limit in probability (if existent). It then follows by Lemma 8.14 that for each $z \in \mathbb{C}$, the Stieltjes transforms $s(z ; \mathbf{X})$ and $s(z ; \mathbf{Y})$ associated with the matrices $\mathbf{W}(\mathbf{X})$ and $\mathbf{W}(\mathbf{Y})$ also have the same limit in probability (if existent). Thus, it remains to identify the limit in the Gaussian case.

From now on, let $\mathbf{A}_{t}$ be defined by $\mathbf{A}_{t}=\left(\left(\mathbf{Y}^{(2)}\right)^{*} \mathbf{Y}^{(2)}+t \mathbf{I}\right)^{-1}$. Then the matrices $\left(\mathbf{Y}^{(1)}\right)^{*} \mathbf{Y}^{(1)}$ and $\mathbf{A}_{t}\left(\mathbf{Y}^{(2)}\right)^{*} \mathbf{Y}^{(2)} \mathbf{A}_{t}$ are asymptotically free. By the Marchenko-Pastur theorem (Theorem 8.1), their limiting (mean) empirical spectral distributions are given by $\varrho$, the Marchenko-Pastur distribution, and $\sigma_{t}$, the induced measure of $\varrho$ under the mapping $x \mapsto(x+t)^{-1} x(x+t)^{-1}$, respectively. Thus, by Lemma A.2, the limiting (mean) 
empirical spectral distribution of $\mathbf{W}_{t}(\mathbf{Y})$ is given by $\varrho \otimes \sigma_{t}$, with $S$-transform $S_{\varrho} \cdot S_{\sigma_{t}}$. Clearly, as $t \rightarrow 0$, we have $\sigma_{t} \rightarrow \sigma$ in Kolmogorov distance, where $\sigma$ denotes the induced measure of $\varrho$ under the mapping $x \mapsto x^{-1}$. Using (5.10) and (5.15), we obtain $\varrho \bigotimes \sigma_{t} \rightarrow \varrho \bigotimes \sigma$ in Kolmogorov distance and $S_{\varrho} \cdot S_{\sigma_{t}} \rightarrow S_{\varrho} \cdot S_{\sigma}$. It therefore follows by Lemma 8.14 that the limiting (mean) empirical spectral distribution of $\mathbf{W}(\mathbf{Y})$ has the $S$-transform $S_{\varrho} \cdot S_{\sigma}$. Now, by Remarks 8.3 and 8.4, we have

$$
S_{\varrho}(z)=\frac{1}{1+z} \quad \text { and } \quad S_{\sigma}(z)=-z
$$

and therefore

$$
\left(S_{\varrho} \cdot S_{\sigma}\right)(z)=-\frac{z}{1+z} .
$$

After a simple calculation we get that the density of the limit distribution is given by

$$
p(x)=\frac{1}{\pi} \frac{1}{\sqrt{x}(1+x)} \mathbb{I}\{x \geq 0\} .
$$

This completes the proof of Theorem 8.13.

\subsubsection{Product of Independent Matrices from Spherical Ensemble}

In this section we consider products of independent matrices of type $\mathbf{X}^{(2 q-1)}\left(\mathbf{X}^{(2 q)}\right)^{-1}$, for $q=1, \ldots, m$, assuming that all matrices and all entries of matrices are independent. Let $\mathbf{F}=\prod_{q=1}^{m} \mathbf{X}^{(2 q-1)}\left(\mathbf{X}^{(2 q)}\right)^{-1}$ and $\mathbf{W}=\mathbf{F F}^{*}$. Let $s_{1}^{2} \geq \ldots \geq s_{n}^{2}$ denote the eigenvalues of the matrix $\mathbf{W}$ and let $\mathcal{G}_{n}(x)$ denote the empirical distribution function

$$
\mathcal{G}_{n}(x)=\frac{1}{n} \sum_{j=1}^{n} \mathbb{I}\left\{s_{j}^{2} \leq x\right\} .
$$

We shall assume as usual that $\mathbf{X}^{(q)}=\frac{1}{\sqrt{n}}\left(X_{j k}^{(q)}\right)$, and that (8.1) or (8.2) holds. Then we have the following result, which was already announced by the first and third author of this paper in [26]. See also Forrester [20] and Forrester and Liu [21] for the Gaussian case. The density in (8.63) also occurs in Biane [12] in the context of free multiplicative Lévy processes.

Theorem 8.15. Assume that the random variables $X_{j k}^{(q)}$, for $q=1, \ldots, 2 m$ and $j, k=$ $1, \ldots, n$ satisfy condition (8.23). Then

$$
\lim _{n \rightarrow \infty} \mathcal{G}_{n}(x)=G_{m}(x) \text { in probability }
$$

where $G_{m}(x)$ denotes the distribution function with density $p_{m}(x)=G_{m}^{\prime}(x)$ given by

$$
p_{m}(x)=\frac{1}{\pi} \frac{\sin \frac{\pi m}{m+1}}{x^{\frac{m}{m+1}}\left(x^{\frac{2}{m+1}}-2 x^{\frac{1}{m+1}} \cos \frac{\pi m}{m+1}+1\right)} .
$$


Similarly as the proof of Theorem 8.13, the proof of Theorem 8.15 is rather technical. Before we can apply Theorem 3.2, we must regularize all the inverse matrices, and this requires a slightly more complicated construction than in the previous subsection.

Let us formulate a general result. Let $\mathbf{A}$ and $\mathbf{B}$ be random matrices of size $n \times n$, and let $\mathbf{X}$ be a Girko-Ginibre matrix of size $n \times n$ satisfying A.3. Introduce the inverse $\mathbf{X}_{0}^{-1}:=\mathbf{X}^{-1}$ and the regularized inverse

$$
\mathbf{X}_{t}^{-1}:=\left(\mathbf{X}^{*} \mathbf{X}+t \mathbf{I}\right)^{-1} \mathbf{X}^{*}=\mathbf{X}^{*}\left(\mathbf{X X}^{*}+t \mathbf{I}\right)^{-1}
$$

For any $t \geq 0$, let $\mathbf{F}_{t}:=\mathbf{A} \mathbf{X}_{t}^{-1} \mathbf{B}, \mathbf{W}_{t}:=\mathbf{F}_{t} \mathbf{F}_{t}^{*}, \mathbf{R}_{t}(z):=\left(\mathbf{W}_{t}-z\right)^{-1}(z \notin \mathbb{R})$ and $g_{t}(z):=\frac{1}{n} \operatorname{Tr} \mathbf{R}_{t}(z)$.

Lemma 8.16 (Regularization Lemma). Let $\mathbf{A}$ and $\mathbf{B}$ be random matrices of size $n \times n$, and let $\mathbf{X}$ be Girko-Ginibre random matrices of size $n \times n$ satisfying (A.3). Suppose that the matrices $\mathbf{B}$ satisfy Conditions (C0), (C1), (C2) and that their squared singular value distributions converge weakly in probability to some probability measure $\nu$. Then, for any $z \notin \mathbb{R}$,

$$
\lim _{t \rightarrow 0} \limsup _{n \rightarrow \infty}\left|g_{t}(z)-g_{0}(z)\right|=0 \quad \text { in probability, }
$$

Even more, the convergence is uniform in $\mathbf{A}$.

Remark. Note that our assumptions on $\mathbf{X}$ and $\mathbf{B}$ imply that

$$
\operatorname{Pr}(\{\mathbf{X} \text { invertible and } \mathbf{B} \text { invertible }\})=1+o(1) \quad \text { as } n \rightarrow \infty .
$$

In the following considerations we always work on this event. (This is possible because we are interested in convergence in probability.) In particular, the inverse $\mathbf{X}_{0}^{-1}$ exists on this event.

Proof. The main idea of the proof is as follows. Firstly, we replace the matrix $\mathbf{B}$ by some regularized version $\mathbf{B}_{s}$ whose singular value distribution is bounded away from zero and infinity. Secondly, we regularize the matrix $\mathbf{X}^{-1}$ as described above. Thirdly, we undo the regularization of the matrix $\mathbf{B}$.

For $x>0$ and $s \in(0,1)$, let

$$
f(s, x):=\frac{\sqrt{x^{2}+s}}{1+s \sqrt{x^{2}+s}}
$$

Note that as $s \rightarrow 0$, we have $f(s, x) \rightarrow f(0, x):=x$ for any $x>0$. Furthermore, note that for each $s>0$, the function $x \mapsto f(s, x)$ is increasing in $x$, with values in the bounded interval $\left(\frac{s^{1 / 2}}{1+s^{3 / 2}}, s^{-1}\right)$. Also, setting $h(s, x):=\log f(s, x)$, we may write

$$
f(s, x)=\exp (h(s, x)) \quad \text { and } \quad \partial_{1} f(s, x)=f(s, x) \partial_{1} h(s, x) .
$$

Here, $\partial_{1}$ denotes the partial derivative w.r.t. the first argument. 
Write $\mathbf{B}=\mathbf{U} \boldsymbol{\Lambda} \mathbf{V}^{*}$ (singular value distribution), and set $\mathbf{B}_{s}:=\mathbf{U} f(s, \boldsymbol{\Lambda}) \mathbf{V}^{*}, s \geq 0$. Here, $f(s, \boldsymbol{\Lambda})$ is obtained by applying $f(s, \cdot)$ to the diagonal elements of $\boldsymbol{\Lambda}$. Note that

$$
\varkappa\left(\mathbf{B}_{s}\right):=\left\|\mathbf{B}_{s}\right\|\left\|\mathbf{B}_{s}^{-1}\right\| \leq 1+s^{-3 / 2}
$$

and

$$
\frac{\partial \mathbf{B}_{s}}{\partial s}=\mathbf{U} f(s, \boldsymbol{\Lambda}) \partial_{1} h(s, \boldsymbol{\Lambda}) \mathbf{V}^{*}=\mathbf{U} f(s, \boldsymbol{\Lambda}) \mathbf{V}^{*} \mathbf{V} \partial_{1} h(s, \boldsymbol{\Lambda}) \mathbf{V}^{*}=: \mathbf{B}_{s} \mathbf{H}_{s} .
$$

Set $\mathbf{F}_{t, s}:=\mathbf{A X}_{t}^{-1} \mathbf{B}_{s}, \mathbf{W}_{t, s}:=\mathbf{F}_{t, s} \mathbf{F}_{t, s}^{*}$ and $\mathbf{R}_{t, s}(z):=\left(\mathbf{W}_{t, s}-z\right)^{-1}(z \notin \mathbb{R})$. Then we may write

$$
\begin{aligned}
\mathbf{R}_{t}(z)-\mathbf{R}_{0}(z) & =\mathbf{R}_{t, 0}(z)-\mathbf{R}_{t, s}(z)+\mathbf{R}_{t, s}(z)-\mathbf{R}_{0, s}(z)+\mathbf{R}_{0, s}(z)-\mathbf{R}_{0,0}(z) \\
& =-\int_{0}^{s} \frac{\partial}{\partial s} \mathbf{R}_{t, u}(z) d u+\int_{0}^{t} \frac{\partial}{\partial t} \mathbf{R}_{u, s}(z) d u+\int_{0}^{s} \frac{\partial}{\partial s} \mathbf{R}_{0, u}(z) d u .
\end{aligned}
$$

It is easy to check that

$$
\frac{\partial}{\partial t} \mathbf{R}_{t, s}=\mathbf{R}_{t s} \mathbf{F}_{t s}\left(\mathbf{B}_{s}^{-1}\left(\mathbf{X X}^{*}+t\right)^{-1} \mathbf{B}_{s}+\mathbf{B}_{s}^{*}\left(\mathbf{X X}^{*}+t\right)^{-1}\left(\mathbf{B}_{s}^{*}\right)^{-1}\right) \mathbf{F}_{t s}^{*} \mathbf{R}_{t s}
$$

and, by (8.52),

$$
\frac{\partial}{\partial s} \mathbf{R}_{t, s}=-2 \mathbf{R}_{t s} \mathbf{F}_{t s} \mathbf{H}_{s} \mathbf{F}_{t s}^{*} \mathbf{R}_{t s} .
$$

Now, for any $n \times n$ matrices $\mathbf{M}_{1}, \mathbf{M}_{2}, \mathbf{M}_{3}$, with $\mathbf{M}_{2}$ self-adjoint, we have

$$
\left|\operatorname{Tr}\left(\mathbf{M}_{1} \mathbf{M}_{2} \mathbf{M}_{3}\right)\right| \leq\left\|\mathbf{M}_{1}\right\|\left\|\mathbf{M}_{3}\right\| \operatorname{Tr}\left|\mathbf{M}_{2}\right|,
$$

where $\left|\mathbf{M}_{2}\right|$ is defined by spectral calculus. Furthermore, for any $t, s \geq 0$, we have

$$
\left\|\mathbf{R}_{t s} \mathbf{F}_{t s}\right\| \leq\left(v^{-1}\left(1+|z| v^{-1}\right)\right)^{1 / 2}, \quad\left\|\mathbf{F}_{t s}^{*} \mathbf{R}_{t s}\right\| \leq\left(v^{-1}\left(1+|z| v^{-1}\right)\right)^{1 / 2} .
$$

It therefore follows from (8.51), (8.54) and (8.55) that

$$
\left|\frac{\partial}{\partial t} \frac{1}{n} \operatorname{Tr} \mathbf{R}_{t, s}\right| \leq 2\left(1+s^{-3 / 2}\right)\left(v^{-1}\left(1+|z| v^{-1}\right)\right) \frac{1}{n} \operatorname{Tr}\left(\mathbf{X X}^{*}+t\right)^{-1} .
$$

and

$$
\left|\frac{\partial}{\partial s} \frac{1}{n} \operatorname{Tr} \mathbf{R}_{t, s}\right| \leq 2\left(v^{-1}\left(1+|z| v^{-1}\right)\right) \frac{1}{n} \operatorname{Tr}\left|\mathbf{H}_{s}\right| .
$$

Taking the normalized trace in (8.53) and using the previous estimates, it follows that for any $s \in(0,1)$,

$$
\begin{aligned}
\left|\frac{1}{n} \operatorname{Tr} \mathbf{R}_{t}(z)-\frac{1}{n} \operatorname{Tr} \mathbf{R}_{0}(z)\right| \leq 2\left(1+s^{-3 / 2}\right)\left(v^{-1}(1\right. & \left.\left.+|z| v^{-1}\right)\right) \int_{0}^{t} \frac{1}{n} \operatorname{Tr}\left(\mathbf{X X}^{*}+u\right)^{-1} d u \\
& +4\left(v^{-1}\left(1+|z| v^{-1}\right)\right) \int_{0}^{s} \frac{1}{n} \operatorname{Tr}\left|\mathbf{H}_{u}\right| d u .
\end{aligned}
$$


We will now show the following:

$$
\begin{gathered}
(\forall \varepsilon>0) \lim _{t \rightarrow 0} \limsup _{n \rightarrow \infty} \operatorname{Pr}\left(\int_{0}^{t} \frac{1}{n} \operatorname{Tr}\left(\mathbf{X} \mathbf{X}^{*}+u\right)^{-1} d u \geq \varepsilon\right)=0 . \\
(\forall \varepsilon>0) \lim _{s \rightarrow 0} \limsup _{n \rightarrow \infty} \operatorname{Pr}\left(\int_{0}^{s} \frac{1}{n} \operatorname{Tr}\left|\mathbf{H}_{u}\right| d u \geq \varepsilon\right)=0 .
\end{gathered}
$$

Once we have (8.58) and (8.59), it is easy to complete the proof. Indeed, fix $\varepsilon>0$. Then, by (8.58), there exists a function $s(t)$ such that $s(t) \rightarrow 0$ as $t \rightarrow 0$ and still

$$
\lim _{t \rightarrow 0} \limsup _{n \rightarrow \infty} \operatorname{Pr}\left(\left(1+s(t)^{-3 / 2}\right) \int_{0}^{t} \frac{1}{n} \operatorname{Tr}\left(\mathbf{X X}^{*}+u\right)^{-1} d u \geq \varepsilon\right)=0 .
$$

Using (8.59), we therefore obtain

$$
\begin{aligned}
\lim _{t \rightarrow 0} \limsup _{n \rightarrow \infty} \operatorname{Pr}\left(\left|\frac{1}{n} \operatorname{Tr} \mathbf{R}_{t}(z)-\frac{1}{n} \operatorname{Tr} \mathbf{R}_{0}(z)\right| \geq 2 \varepsilon\right) \\
\leq \lim _{t \rightarrow 0} \limsup _{n \rightarrow \infty} \operatorname{Pr}\left(2\left(1+s(t)^{-3 / 2}\right)\left(v^{-1}\left(1+|z| v^{-1}\right)\right) \int_{0}^{t} \frac{1}{n} \operatorname{Tr}\left(\mathbf{X X}^{*}+u\right)^{-1} d u \geq \varepsilon\right) \\
\quad+\lim _{t \rightarrow 0} \limsup _{n \rightarrow \infty} \operatorname{Pr}\left(4\left(v^{-1}\left(1+|z| v^{-1}\right)\right) \int_{0}^{s(t)} \frac{1}{n} \operatorname{Tr}\left|\mathbf{H}_{u}\right| d u \geq \varepsilon\right)=0,
\end{aligned}
$$

i.e. the desired conclusion.

Thus, it remains to show (8.58) and (8.59). We have already checked that (8.58) follows from our assumption that $\mathbf{X}$ satisfies Conditions (C0), (C1) and (C2); see the proof of Lemma 8.14. It is straightforward to check that

$$
\partial_{1} h(s, x)=\frac{1-2\left(x^{2}+s\right)^{3 / 2}}{2\left(x^{2}+s\right)\left(1+s\left(x^{2}+s\right)^{1 / 2}\right)} .
$$

Since $s<1$, it follows that

$$
\left|\partial_{1} h(s, x)\right| \leq \frac{C}{s+x^{2}} \quad(x \leq 1) \quad \text { and } \quad\left|\partial_{1} h(s, x)\right| \leq \frac{C x}{1+s x} \quad(x \geq 1) .
$$

We therefore obtain

$$
\begin{aligned}
& \int_{0}^{s} \frac{1}{n} \operatorname{Tr}\left|\mathbf{H}_{u}\right| d u \leq \int_{0}^{s} \frac{1}{n} \sum_{k=1}^{n}\left|\partial_{1} h\left(u, s_{k}(\mathbf{B})\right)\right| d u \\
& \quad \leq \frac{C}{n} \sum_{k: s_{k}(\mathbf{B}) \leq 1} \int_{0}^{s} \frac{1}{u+s_{k}^{2}(\mathbf{B})} d u+\frac{C}{n} \sum_{k: s_{k}(\mathbf{B}) \geq 1} \int_{0}^{s} \frac{s_{k}(\mathbf{B})}{1+u s_{k}(\mathbf{B})} d u \\
& \quad \leq \frac{C}{n} \sum_{k: s_{k}(\mathbf{B}) \leq 1}\left(\log \left(s+s_{k}^{2}(\mathbf{B})\right)-\log \left(s_{k}^{2}(\mathbf{B})\right)\right)+\frac{C}{n} \sum_{k: s_{k}(\mathbf{B}) \geq 1} \log \left(1+s s_{k}(\mathbf{B})\right) .
\end{aligned}
$$

Since the matrix B satisfies (C0), (C1), (C2) and the squared singular value distribution of $\mathbf{B}$ is weakly convergent to a limit $\nu$ with $\int|\log | d \nu<\infty$ (by Lemma A.9 in the appendix), this implies (8.59). 
We now return to Theorem 8.15 .

Proof of Theorem 8.15. The proof is by induction on $m$. Suppose that $m>1$ and that the result is true of all smaller values of $m$. (The case of a single spherical matrix is Theorem 8.13,) We start with a regularisation of the inverse matrices. We introduce the following matrices

$$
\left(\mathbf{X}^{(2 q)}\right)_{0}^{-1}=\left(\mathbf{X}^{(2 q)}\right)^{-1} \quad \text { and } \quad\left(\mathbf{X}^{(2 q)}\right)_{t}^{-1}=\left(\mathbf{X}^{(2 q)^{*}} \mathbf{X}^{(2 q)}+t \mathbf{I}\right)^{-1} \mathbf{X}^{(2 q)^{*}} .
$$

Let $\mathbf{F}_{t}=\prod_{q=1}^{m}\left(\mathbf{X}^{(2 q-1)}\left(\mathbf{X}^{(2 q)}\right)_{t}^{-1}\right)$ and $\mathbf{W}_{t}=\mathbf{F}_{t} \mathbf{F}_{t}^{*}, t \geq 0$. Also, let $\mathbf{R}_{t}(z):=\left(\mathbf{W}_{t}-z \mathbf{I}\right)^{-1}$ and $s_{t}(z):=\frac{1}{n} \operatorname{Tr} \mathbf{R}_{t}(z), t \geq 0, z \in \mathbb{C}^{+}$.

\section{Lemma 8.17.}

$$
\lim _{t \rightarrow 0} \limsup _{n \rightarrow \infty}\left|s_{t}(z)-s_{0}(z)\right|=0 \quad \text { in probability }
$$

Proof. We apply Lemma 8.16. Let $\mathbf{Z}_{t}^{(k)}:=\mathbf{X}^{(2 k-1)}\left(\mathbf{X}^{(2 k)}\right)_{t}^{-1}, k=1, \ldots, m$ and

$$
s\left(t_{1}, \ldots, t_{m} ; z\right):=\frac{1}{n} \operatorname{Tr}\left(\left(\mathbf{Z}_{t_{1}}^{(1)} \cdots \mathbf{Z}_{t_{m}}^{(m)}\right)\left(\mathbf{Z}_{t_{1}}^{(1)} \cdots \mathbf{Z}_{t_{m}}^{(m)}\right)^{*}-z \mathbf{I}\right)^{-1} .
$$

Clearly, writing $\mathbf{v}_{k}:=(t, \ldots, t, 0, \ldots, 0)$ for the vector consisting of $k t$ 's and $m-k 0$ 's, we have

$$
\left|s_{t}(z)-s_{0}(z)\right| \leq \sum_{k=1}^{n}\left|s_{k}\left(\mathbf{v}_{k} ; z\right)-s_{k}\left(\mathbf{v}_{k-1} ; z\right)\right| .
$$

By Lemma 8.16, each of the summands converges to zero in probability. Here we use (i) the inductive hypothesis and (ii) the fact that an arbitrary product of independent spherical matrices satisfies Conditions $(\mathrm{C} 0)-(\mathrm{C} 2)$. The latter will be checked in the proof of Theorem 8.24, note that the verification does not rely on the results in this section. This completes the proof of Lemma 8.17 .

We continue with the proof of Theorem 8.15. We may now use Theorem 3.2 for the matrix $\mathbf{W}_{t}$. The Lindeberg condition (3.1) follows from condition (8.23). The check of the remaining conditions of Theorem 3.2 is similar to that in the previous subsection; we omit the details. Thus, by a similar argument as in the proof of Theorem 8.13 (but with Lemma 8.17 instead of Lemma 8.14), it remains to identify the limiting empirical spectral distribution of the matrix $\mathbf{W}(\mathbf{Y})$ in the Gaussian case.

Here we can use the same approach and notation as in the proof of Theorem 8.13 , Firstly, by asymptotic freeness, Lemma A.2 and Theorem 8.1, the limiting (mean) empirical spectral distribution of the matrices $\mathbf{W}_{t}(\mathbf{Y})$ is given by $\varrho^{\square m} \nabla \sigma_{t}^{\nabla m}$, with corresponding $S$-transform $S_{\mathbf{W}_{t}}(z)=S_{\varrho}^{m}(z) \cdot S_{\sigma_{t}}^{m}(z)$. Secondly, using Lemma 8.17, it follows that the limiting (mean) empirical spectral distribution of the matrices $\mathbf{W}(\mathbf{Y})$ is given by $\varrho^{\bigotimes m} \nabla \sigma^{\bigotimes m}$, with corresponding $S$-transform $S_{\mathbf{W}}(z)=S_{\varrho}^{m}(z) \cdot S_{\sigma}^{m}(z)$. Thirdly, by Remarks 8.3 and 8.4 , we get

$$
S_{\mathbf{W}}(z)=(-1)^{m} \frac{z^{m}}{(z+1)^{m}}
$$


Using the representation (8.62) we may now determine the Stieltjes transform of the asymptotic distribution of the eigenvalues of the matrix $\mathbf{W}$ and from here determine the density of its distribution. Let $g(z)$ denote the Stieltjes transform of the asymptotic distribution of the eigenvalues of matrix W. By definition of the $S$-transform, we have

$$
S_{\mathbf{W}}(-(1+z g(z)))=\frac{g(z)}{1+z g(z)} .
$$

Combining the last two equalities, we get

$$
(-1)^{m} \frac{(1+z g(z))^{m}}{(z g(z))^{m}}=\frac{g(z)}{1+z g(z)} .
$$

Solving the last equation, we obtain

$$
g(z)=-\frac{1}{z-(-1)^{\frac{m}{m+1}} z^{\frac{m}{m+1}}} .
$$

Since $\operatorname{Im} g(z) \geq 0$ we may take here the $\operatorname{root}(-1)^{\frac{m}{m+1}}=\cos \frac{\pi m}{m+1}-i \sin \frac{\pi m}{m+1}$. The density of the distribution of $F_{m}(x)$ satisfies the equality

$$
\left.p_{m}(x)=F_{m}^{\prime}(x)=\frac{1}{\pi} \lim _{v \rightarrow 0} \operatorname{Im} g(x+i v)\right)=\frac{\sin \frac{\pi m}{m+1}}{\pi x^{\frac{m}{m+1}}\left(x^{\frac{2}{m+1}}-2 x^{\frac{1}{m+1}} \cos \frac{\pi m}{m+1}+1\right)} .
$$

For $m=2$, we have

$$
p_{2}(x)=\frac{\sqrt{3}}{2 \pi x^{\frac{2}{3}}\left(x^{\frac{2}{3}}+x^{\frac{1}{3}}+1\right)} .
$$

Thus Theorem 8.13 is proved completely.

\subsection{Applications of Theorem 4.4: Distribution of eigenvalues}

In this section we consider applications of Theorem 4.4. These applications rely on the previous applications of Theorem 3.2. Actually, we need the universality of the limiting singular value distribution not only for the matrices $\mathbf{F}_{\mathbf{X}}$ and $\mathbf{F}_{\mathbf{Y}}$, but also for all the shifted matrices $\mathbf{F}_{\mathbf{X}}-\alpha \mathbf{I}$ and $\mathbf{F}_{\mathbf{Y}}-\alpha \mathbf{I}$, with $\alpha \in \mathbb{C}$. However, since these extensions are more and less straightforward, we omit the details.

\subsubsection{Circular law}

Let $\mathbf{X}$ denote the $n \times n$ random matrix with independent entries $\frac{1}{\sqrt{n}} X_{j k}$ such that (8.1) or (8.2) holds. Assume that the r.v.'s $X_{j k}$ satisfy the condition

$$
\max _{j, k} \mathbf{E}\left|X_{j k}\right|^{2} \mathbb{I}\left\{\left|X_{j k}\right|>M\right\} \rightarrow 0, \quad \text { as } \quad M \rightarrow \infty .
$$

Let $\lambda_{1}, \ldots, \lambda_{n}$ denote the eigenvalues of the matrix $\mathbf{X}$. Denote by $\mu_{n}$ the empirical spectral distribution of the matrix $\mathbf{X}$. Then we have the following result, cf. Girko [22], Bai [7], Pan and Zhou [35], Götze and Tikhomirov [24] as well as Tao and Vu [41]. 
Theorem 8.18. Assume that condition (8.64) holds. Then the measures $\mu_{n}$ converge weakly in probability to the uniform distribution $\mu$ on the unit disc.

Proof. To prove Theorem 8.18, we first apply Theorem 4.4. Then we compute the limit distribution for the Gaussian case using the result of Theorem 7.6.

Note that condition (3.1) is implied by condition (8.64) and that conditions (3.2) and (3.20) - 3.25) of Theorem 3.2 have been checked in Subsection 8.1.1. It remains to check conditions $(\mathrm{C} 0),(\mathrm{C} 1)$ and $(\mathrm{C} 2)$ of Theorem 4.4. To this end we can use existing bounds for singular values; see Lemmas A.4, A.5 and A.6 in Appendix A.3. We may therefore apply Theorem 4.4.

Let $\mathbf{V}=\mathbf{V}_{\mathbf{Y}}$ be defined as in Section 6, with $\mathbf{F}=\mathbf{F}_{\mathbf{Y}}=\mathbf{Y}$. By Proposition 5.8, the matrices $\mathbf{V}$ and $\mathbf{J}(\alpha)$ are asymptotically free. Furthermore, it follows from Theorem 8.1 that the limiting eigenvalue distribution of the matrices $\mathbf{V}_{\mathbf{Y}}$ is given by the semicircular law. We now compute the limiting eigenvalue distribution of the matrices $\mathbf{F}_{\mathbf{Y}}$ using Theorem [7.6. It is well-known, see e.g. Rao and Speicher [37, Section 3, that

$$
S_{\mathbf{V}}(z)=-\frac{1}{\sqrt{z}}
$$

Thus, the equations (7.9) read

$$
\begin{aligned}
\psi(\alpha)(1-\psi(\alpha)) & =|\alpha|^{2} \varkappa(\alpha)^{2}, \\
\varkappa(\alpha) & =\sqrt{1-\psi(\alpha)} .
\end{aligned}
$$

Solving these equations, we get

$$
\psi(\alpha)=1, \varkappa(\alpha)=0 \quad \text { or } \quad \psi(\alpha)=|\alpha|^{2}, \varkappa(\alpha)=\sqrt{1-|\alpha|^{2}} .
$$

Now recall that (i) $\psi(\alpha) \in[0,1]$ by Theorem 4.4, (ii) $\varkappa$ is continuous by Remark 6.5, and (iii) $\varkappa(\alpha) \neq 0$ for $\alpha \approx 0$ by Lemma 6.6. Thus, we obtain a unique solution, namely

$$
\begin{array}{r}
\psi= \begin{cases}|\alpha|^{2}, & u^{2}+v^{2} \leq 1, \\
1, & u^{2}+v^{2}>1,\end{cases} \\
\varkappa= \begin{cases}\sqrt{1-|\alpha|^{2},} & u^{2}+v^{2} \leq 1, \\
0, & u^{2}+v^{2}>1 .\end{cases}
\end{array}
$$

It follows from here that

$$
u \frac{\partial \psi}{\partial u}+v \frac{\partial \psi}{\partial v}= \begin{cases}2|\alpha|^{2}, & u^{2}+v^{2} \leq 1, \\ 0, & u^{2}+v^{2}>1 .\end{cases}
$$

Using (7.10), it therefore follows that the density $f(u, v)$ of the limiting empirical spectral distribution of the matrix $\mathbf{Y}$ is given by the equality

$$
f(u, v)= \begin{cases}\frac{1}{\pi}, & u^{2}+v^{2} \leq 1 \\ 0, & u^{2}+v^{2}>1 .\end{cases}
$$




\subsubsection{Product of Independent Square Matrices}

Let $m \geq 1$. Consider independent random matrices $\mathbf{X}^{(q)}, q=1, \ldots, m$ with independent entries $\frac{1}{\sqrt{n}} X_{j k}^{(q)}, 1 \leq j, k \leq n, q=1, \ldots, m$, and suppose that (8.1) or (8.2) holds. Let $\mathbf{F}=\prod_{q=1}^{m} \mathbf{X}^{(q)}$. Then we have the following result, cf. Burda, Janik and Waclaw [15] for the Gaussian case and Götze and Tikhomirov [25] as well as O'Rourke and Soshnikov 38] for the general case.

Theorem 8.19. Let the r.v.'s $X_{j k}^{(q)}$ satisfy the condition

$$
\max _{1 \leq q \leq m} \max _{j, k \geq 1} \mathbf{E}\left|X_{j k}^{(q)}\right|^{2} \mathbb{I}\left\{\left|X_{j k}^{(q)}\right|>M\right\} \rightarrow 0, \quad \text { as } \quad M \rightarrow \infty .
$$

Then the empirical spectral distributions $\mu_{n}$ of the matrices $\mathbf{F}$ converge weakly in probability to the measure $\mu$ with Lebesgue density

$$
f(u, v)=\frac{1}{m \pi\left(u^{2}+v^{2}\right)^{\frac{m-1}{m}}} \mathbb{I}\left\{u^{2}+v^{2} \leq 1\right\} .
$$

Remark 8.20. The limiting measure $\mu$ is the induced measure of the uniform distribution on the unit disc under the mapping $z \mapsto z^{m}$. Consequently, the product of $m$ independent square matrices has the same limiting empirical spectral distribution as the $m$ th power of a single matrix.

Proof. The conditions of Theorem 3.2 were checked in the proof of Theorem 8.1.2. The condition $(C 0)$ of Theorem 4.4 for $p=2$ follows from Lemma 7.2 in [25], where it is shown that

$$
\frac{1}{n} \mathbf{E}\|\mathbf{F}\|_{2}^{2} \leq C
$$

Furthermore, in Lemma 5.1 in [25] it is proved that there exist positive constants $Q$ and $A$ such that

$$
\operatorname{Pr}\left\{s_{n}(\mathbf{F}-\alpha \mathbf{I}) \leq n^{-Q}\right\} \leq C n^{-A} .
$$

This implies condition $(C 1)$ of Theorem 4.4. Moreover, inequality (5.16) and Lemma 5.2 in 25] together imply that for some $0<\gamma<1$, for any sequence $\delta_{n} \rightarrow 0$

$$
\lim _{n \rightarrow \infty} \frac{1}{n} \sum_{k=n_{1}}^{n_{2}}\left|\log s_{k}(\mathbf{F}-\alpha \mathbf{I})\right|=0,
$$

with $n_{1}=\left[n-n \delta_{n}\right]+1$ and $n_{2}=\left[n-n^{\gamma}\right]$. This implies condition $(C 2)$ of Theorem 4.4 According to Theorem 4.4 we may now consider the Gaussian matrices $\mathbf{Y}^{(q)}, q=1, \ldots, m$. Let

$$
\mathbf{V}=\left[\begin{array}{cc}
\mathbf{O} & \mathbf{F}_{\mathbf{Y}} \\
\mathbf{F}_{\mathbf{Y}}^{*} & \mathbf{O}
\end{array}\right]
$$


By Proposition 5.8, the matrices $\mathbf{V}$ and $\mathbf{J}(\alpha)$ are asymptotically free. Moreover, it follows from Remark 8.3 and Lemma A.2 that the $S$-transform corresponding to the matrices $\mathbf{W}:=\mathbf{F}_{\mathbf{Y}} \mathbf{F}_{\mathbf{Y}}^{*}$ is given by

$$
S_{\mathbf{W}}(z)=\left(\frac{1}{z+1}\right)^{m}
$$

Thus, the $S$-transform of the matrix $\mathbf{V}$ is given by the formula

$$
S_{\mathbf{V}}(z)=-\frac{1}{\sqrt{z}(1+z)^{\frac{m-1}{2}}} .
$$

We rewrite equations (7.9) for this case:

$$
\begin{gathered}
\psi(\alpha)(1-\psi(\alpha))=|\alpha|^{2} \varkappa^{2}(\alpha), \\
\varkappa(\alpha)=\sqrt{1-\psi(\alpha)}(\psi(\alpha))^{-\frac{m-1}{2}} .
\end{gathered}
$$

Solving this system we find by similar arguments as in the previous subsection that

$$
\begin{aligned}
& \psi(\alpha)= \begin{cases}|\alpha|^{\frac{2}{m}}, & u^{2}+v^{2} \leq 1, \\
1, & u^{2}+v^{2}>1,\end{cases} \\
& \varkappa(\alpha)= \begin{cases}|\alpha|^{-\frac{m-1}{m}} \sqrt{1-|\alpha|^{\frac{2}{m}}}, & u^{2}+v^{2} \leq 1, \\
0, & u^{2}+v^{2}>1,\end{cases}
\end{aligned}
$$

and, for $u^{2}+v^{2} \leq 1$,

$$
u \frac{\partial \psi}{\partial u}+v \frac{\partial \psi}{\partial v}=\frac{2|\alpha|^{\frac{2}{m}}}{m} .
$$

By (17.10), these relations immediately imply that

$$
f(x, y)= \begin{cases}\frac{1}{\pi m\left(x^{2}+y^{2}\right)^{\frac{m-1}{m}}}, & x^{2}+y^{2} \leq 1 \\ 0, & x^{2}+y^{2}>1\end{cases}
$$

and Theorem 8.19 is proved.

\subsubsection{Product of Independent Rectangular Matrices}

Let $m \geq 1$ be fixed. Let for any $n \geq 1$ be given integers $n_{0}=n, n_{1} \geq n, \ldots, n_{m-1} \geq n$ and $n_{m}=n$. Assume that $y_{q}=\lim _{n \rightarrow \infty} \frac{n}{n_{q}} \in(0,1], q=1, \ldots, m$. Note that $y_{m}=1$. Consider independent random matrices $\mathbf{X}^{(q)}$ of order $n_{q-1} \times n_{q}, q=1, \ldots, m$, with independent entries $\frac{1}{\sqrt{n_{q}}} X_{j k}^{(q)}$ as in (8.1) or (8.2). Put $\mathbf{F}=\prod_{q=1}^{m} \mathbf{X}^{(q)}$. Then we have the following result, see also Burda, Jarosz, Livan, Nowak, Swiech [16] and Tikhomirov [43] for related results in the Gaussian case and in the general case, respectively. 
Theorem 8.21. Assume that the r.v.'s $X_{j k}^{(q)}$ for $q=1, \ldots, m$, and $j=1, \ldots, p_{q-1}$, $k=1, \ldots, p_{q}$ satisfy the condition (8.65). Then the empirical spectral distributions of the matrices $\mathbf{F}$ weakly converge in probability to the measure $\mu$ with Lebesgue density

$$
f(u, v)=\frac{1}{2 \pi|\alpha|^{2}}\left(u \frac{\partial \psi}{\partial u}+v \frac{\partial \psi}{\partial v}\right) \mathbb{I}\left\{u^{2}+v^{2} \leq 1\right\}
$$

where $\alpha=u+i v$ and $\psi(\alpha)$ is given by the unique solution in the interval $[0,1]$ to the equation

$$
\psi(\alpha) \prod_{\nu=1}^{m-1}\left(1-y_{\nu}+y_{\nu} \psi(\alpha)\right)=|\alpha|^{2}, \quad|\alpha| \leq 1 .
$$

In the case $m=2$ this is

$$
f(u, v)=\frac{1}{\pi \sqrt{\left(1-y_{1}\right)^{2}+4\left(u^{2}+v^{2}\right) y_{1}}} \mathbb{I}\left\{u^{2}+v^{2} \leq 1\right\} .
$$

Proof. The conditions of Theorem 3.2 were checked in Subsubsection 8.1.2, The conditions $(C 0),(C 1)$ and $(C 2)$ of Theorem 4.4 may be checked similarly as in the proof of the previous Theorem; we omit the details. To compute the limit measure $\mu$ in the Gaussian case, we may use Theorem 7.6 now. Using Remark 8.3 and Lemma A.2, we may show that

$$
S_{\mathbf{V}}(z)=-\frac{1}{\sqrt{z}} \prod_{\nu=1}^{m-1} \frac{1}{\sqrt{1+y_{\nu} z}}
$$

We have used here that $y_{m}=1$. Inserting (8.70) into (7.9), we get

$$
\begin{aligned}
\psi(\alpha)(1-\psi(\alpha)) & =|\alpha|^{2} \varkappa(\alpha)^{2}, \\
\varkappa(\alpha) & =\sqrt{1-\psi(\alpha)} \prod_{\nu=1}^{m-1} \frac{1}{\sqrt{1-y_{\nu}+y_{\nu} \psi(\alpha)}} .
\end{aligned}
$$

Solving this system we find that for $|\alpha| \leq 1$,

$$
\psi(\alpha) \prod_{\nu=1}^{m-1}\left(1-y_{\nu}+y_{\nu} \psi(\alpha)\right)=|\alpha|^{2} \quad \text { and } \quad \varkappa(\alpha)=0
$$

while for $|\alpha|>1$,

$$
\psi(\alpha)=1 \quad \text { and } \quad \varkappa(\alpha)=0 .
$$

We have used here that the function $h(x):=x \prod_{\nu=1}^{m-1}\left(1-y_{\nu}+y_{\nu} x\right)$ is strictly increasing on $[0, \infty)$ with $h(0)=0$ and $h(1)=1$. Now (8.69) follows immediately from (7.10). In order to check that $f$ is in fact a probability density, regard $\psi$ and $f$ as functions of $r:=\sqrt{u^{2}+v^{2}}$. Then

$$
f(r)=\frac{\psi^{\prime}(r)}{2 \pi r}, \quad 0<r<1,
$$


and it follows using polar coordinates that

$$
\int_{\left\{u^{2}+v^{2}<1\right\}} f(u, v) d u d v=\int_{0}^{1} 2 \pi r f(r) d r=\int_{0}^{1} \psi^{\prime}(r) d r=\psi(1)-\psi(0)=1 .
$$

Finally, for $m=2$ and $u^{2}+v^{2} \leq 1$, we get

$$
\psi(\alpha)\left(1-y_{1}+y_{1} \psi(\alpha)\right)=|\alpha|^{2}
$$

and therefore

$$
\psi(\alpha)=\frac{-\left(1-y_{1}\right)+\sqrt{\left(1-y_{1}\right)^{2}+4|\alpha|^{2} y_{1}}}{2 y_{1}} .
$$

Hence, on the set $\left\{u^{2}+v^{2} \leq 1\right\}$, we obtain

$$
f(u, v)=\frac{1}{2 \pi|\alpha|^{2}}\left(u \frac{\partial \psi}{\partial u}+v \frac{\partial \psi}{\partial v}\right)=\frac{1}{\pi \sqrt{\left(1-y_{1}\right)^{2}+4|\alpha|^{2} y_{1}}} .
$$

Theorem 8.21 is proved.

\subsubsection{Spherical Ensemble}

Let $\mathbf{X}^{(1)}$ and $\mathbf{X}^{(2)}$ be independent $n \times n$ random matrices with independent entries $\frac{1}{\sqrt{n}} X_{j k}^{(q)}$ such that (8.1) or (8.2) holds. Consider the matrix $\mathbf{F}_{\mathbf{X}}=\mathbf{X}^{(1)}\left(\mathbf{X}^{(2)}\right)^{-1}$. Let $\mu_{n}$ denote the empirical spectral distribution of the matrix $\mathbf{F}_{\mathbf{X}}$. Then we have the following result, cf. Bordenave [13].

Theorem 8.22. Let the r.v.'s $X_{j k}^{(q)}$ for $q=1,2$ and $j, k=1, \ldots, n$ satisfy the condition (8.65). Then the measures $\mu_{n}$ weakly converge in probability to the measure $\mu$ with Lebesgue density

$$
f(x, y)=\frac{1}{\pi\left(1+\left(x^{2}+y^{2}\right)\right)^{2}}
$$

Remark 8.23. This density corresponds after stereographic projection of the complex plane to the uniform distribution on the sphere.

Proof. We use Remark 4.5. By Theorem 8.13, for each $\alpha \in \mathbb{C}$, the empirical singular value distributions of the matrices $\mathbf{F}_{\mathbf{X}}-\alpha \mathbf{I}$ have the same weak limit in probability as those of the matrices $\mathbf{F}_{\mathbf{Y}}-\alpha \mathbf{I}$, where $\mathbf{F}_{\mathbf{Y}}=\mathbf{Y}^{(1)}\left(\mathbf{Y}^{(2)}\right)^{-1}$ and $\mathbf{Y}^{(1)}$ and $\mathbf{Y}^{(2)}$ are independent random matrices with independent Gaussian entries. We check the conditions $(C 0),(C 1)$, $(C 2)$. Fix $\alpha \in \mathbb{C}$, and write $\mathbf{F}$ instead of $\mathbf{F}_{\mathbf{X}}$. We start with the condition $(C 0)$ for some $p<\frac{1}{9}$. According to Theorem 3.3.14, c) in [28], we have

$$
\frac{1}{n} \sum_{k=1}^{n} s_{k}^{p}(\mathbf{F}-\alpha \mathbf{I}) \leq \frac{1}{n} \sum_{k=1}^{n} s_{k}^{p}\left(\mathbf{X}^{(1)}-\alpha \mathbf{X}^{(2)}\right) s_{k}^{p}\left(\left(\mathbf{X}^{(2)}\right)^{-1}\right) .
$$


Applying Hölder inequality, we get

$$
\frac{1}{n} \sum_{k=1}^{n} s_{k}^{p}(\mathbf{F}-\alpha \mathbf{I}) \leq\left(\frac{1}{n} \sum_{k=1}^{n} s_{k}^{2}\left(\mathbf{X}^{(1)}-\alpha \mathbf{X}^{(2)}\right)\right)^{\frac{p}{2}}\left(\frac{1}{n} \sum_{k=1}^{n} s_{k}^{-\frac{2 p}{2-p}}\left(\mathbf{X}^{(2)}\right)\right)^{\frac{2-p}{2}} .
$$

Note that

$$
\mathbf{E}\left(\frac{1}{n} \sum_{k=1}^{n} s_{k}^{2}\left(\mathbf{X}^{(1)}-\alpha \mathbf{X}^{(2)}\right)\right) \leq 1+|\alpha|
$$

This implies that the first factor on the r.h.s. of (8.71) is bounded in probability. For $p<\frac{2}{9}$ we have $\beta=\frac{2 p}{2-p}<\frac{1}{4}$. Denote by $\mathcal{G}_{n}(x)$ the empirical distribution function of the squared singular values of the matrix $\mathbf{X}^{(2)}$. By the Marchenko-Pastur theorem

$$
\lim _{n \rightarrow \infty} \varkappa_{n}:=\lim _{n \rightarrow \infty} \sup _{x}\left|\mathcal{G}_{n}(x)-G(x)\right|=0 \quad \text { in probability }
$$

where $G(x)$ has Lebesgue density $g(x)=G^{\prime}(x)=\frac{\sqrt{4-x}}{2 \pi \sqrt{x}} \mathbb{I}\{0<x \leq 4\}$. Furthermore, let $0<\gamma<1$ be as in Lemma A.8, and let $n_{1}=\left[n-n^{\gamma}\right]$ and $n_{2}=\min \left\{n_{1},\left[n\left(1-\varkappa_{n}\right)\right]\right\}$. We have the following decomposition

$$
\frac{1}{n} \sum_{k=1}^{n} s_{k}^{-\beta}\left(\mathbf{X}^{(2)}\right)=\frac{1}{n} \sum_{k=n_{1}+1}^{n} s_{k}^{-\beta}\left(\mathbf{X}^{(2)}\right)+\frac{1}{n} \sum_{k=n_{2}+1}^{n_{1}} s_{k}^{-\beta}\left(\mathbf{X}^{(2)}\right)+\frac{1}{n} \sum_{k=1}^{n_{2}} s_{k}^{-\beta}\left(\mathbf{X}^{(2)}\right) .
$$

By Lemma A.5, we have

$$
\lim _{n \rightarrow \infty} \operatorname{Pr}\left\{s_{k}\left(\mathbf{X}^{(2)}\right) \leq n^{-Q}\right\}=0 .
$$

This implies that for $p<\frac{2(1-\gamma)}{2 Q+1-\gamma}$

$$
\frac{1}{n} \sum_{k=n_{1}+1}^{n} s_{k}^{-\beta}\left(\mathbf{X}^{(2)}\right) \rightarrow 0 \quad \text { as } \quad n \rightarrow \infty \quad \text { in probability. }
$$

Furthermore, by Lemma A.8, we have

$$
\operatorname{Pr}\left\{s_{k}^{-\beta}\left(\mathbf{X}^{(2)}\right)>c\left(\frac{n-k}{n}\right)^{-\beta} \text { for some } k=1, \ldots, n_{1}\right\} \leq \exp \{-c n\} .
$$

Since $\beta<1$, this implies that

$$
\operatorname{Pr}\left\{\frac{1}{n} \sum_{k=n_{2}+1}^{n_{1}} s_{k}^{-\beta}\left(\mathbf{X}^{(2)}\right)>c^{\prime}\left(\frac{n-n_{2}}{n}\right)^{1-\beta}\right\} \leq \exp \left\{-c^{\prime} n\right\} .
$$

From $\frac{n-n_{2}}{n} \leq \varkappa_{n}$ it follows that

$$
\lim _{n \rightarrow \infty} \frac{1}{n} \sum_{k=n_{2}+1}^{n_{1}} s_{k}^{-\beta}\left(\mathbf{X}^{(2)}\right)=0 \quad \text { in probability. }
$$


It remains to prove that the last summand on the r.h.s. of $(8.72)$ is bounded in probability. Again by Lemma A.8, with probability $1-o(1)$, we have $s_{n_{2}}\left(\mathbf{X}^{(2)}\right) \geq c \varkappa_{n}$ and therefore

$$
\frac{1}{n} \sum_{k=1}^{n_{2}} s_{k}^{-\beta}\left(\mathbf{X}^{(2)}\right) \leq\left|\int_{c \varkappa_{n}}^{\infty} x^{-\beta / 2} d\left(\mathcal{G}_{n}(x)-G(x)\right)\right|+\int_{0}^{4} x^{-\beta / 2} d G(x) .
$$

The last integral on the r.h.s. of (8.74) is bounded for $\beta<1$. Integrating by parts in the first integral on the r.h.s. of (8.74), we get

$$
\begin{aligned}
\left|\int_{c \varkappa_{n}}^{\infty} x^{-\beta} d\left(\mathcal{G}_{n}(x)-G(x)\right)\right| & \leq C \varkappa_{n}^{-\beta}\left|\mathcal{G}_{n}\left(\varkappa_{n}\right)-G\left(\varkappa_{n}\right)\right|+\int_{c \varkappa_{n}}^{\infty} \beta y^{-\beta-1}\left|\mathcal{G}_{n}(y)-G(y)\right| d y \\
& \leq C \varkappa_{n}^{1-\beta} .
\end{aligned}
$$

The last inequalities imply that the last summand on the r.h.s. of (8.72) is bounded in probability. This concludes the proof of the condition $(C 0)$. The condition $(C 1)$ follows from the bound

$$
s_{n}(\mathbf{F}-\alpha \mathbf{I}) \geq s_{n}\left(\mathbf{X}^{(1)}-\alpha \mathbf{X}^{(2)}\right) s_{1}^{-1}\left(\mathbf{X}^{(2)}\right)
$$

and Lemmas A.4 and A.7. To prove the condition $(C 2)$, fix a sequence $\left(\delta_{n}\right)$ with $\delta_{n} \geq n^{-\gamma}$ for all $n$ and $\delta_{n} \rightarrow 0$, and let $n_{2}:=n\left[1-\delta_{n}\right]$. Then, by the arguments for condition $(C 1)$ as well as Theorem 3.3.4 in [28], we have, with probability $1-o(1)$,

$$
\begin{aligned}
& \lim _{n \rightarrow \infty} \frac{1}{n} \sum_{k=n_{2}+1}^{n_{1}}\left|\log s_{k}(\mathbf{F}-\alpha \mathbf{I})\right| \\
& \quad \leq \lim _{n \rightarrow \infty} \frac{1}{n} \sum_{k=n_{2}+1}^{n_{1}}\left|\log s_{k}\left(\mathbf{X}^{(1)}-\alpha \mathbf{X}^{(2)}\right)\right|+\lim _{n \rightarrow \infty} \frac{1}{n} \sum_{k=n_{2}+1}^{n_{1}}\left|\log s_{n-k+1}\left(\mathbf{X}^{(2)}\right)\right| .
\end{aligned}
$$

Note that both sums on the r.h.s. converge to zero in probability. For the first sum, this follows from Lemma A.8 applied to the matrix $\mathbf{X}^{(1)}-\alpha \mathbf{X}^{(2)}$, while for the second sum, this follows from the observation that we have, with probability $1-o(1)$,

$$
\begin{aligned}
\frac{1}{n} \sum_{k=n_{2}+1}^{n_{1}}\left|\log s_{n-k+1}\left(\mathbf{X}^{(2)}\right)\right| & \leq \frac{1}{n} \sum_{k=1}^{n-n_{2}}\left|\log s_{k}\left(\mathbf{X}^{(2)}\right)\right| \\
& \leq \frac{1}{n} \sum_{\substack{1 \leq k \leq n-n_{2} \\
s_{k} \leq \delta_{n}^{-1}}}\left|\log s_{k}\left(\mathbf{X}^{(2)}\right)\right|+\int_{\delta_{n}^{-1}}^{\infty} \log x d \mathcal{G}(x) \\
& \leq \frac{n-n_{2}}{n}\left|\log \delta_{n}\right|+\delta_{n}^{2}\left|\log \delta_{n}\right| \frac{1}{n} \sum_{k=1}^{n} s_{k}^{2}\left(\mathbf{X}^{(2)}\right) \\
& \leq \delta_{n}\left|\log \delta_{n}\right|\left(1+\frac{1}{n}\left\|\mathbf{X}^{(2)}\right\|_{2}^{2}\right) .
\end{aligned}
$$

Combining these estimates, we come to the conclusion that $\frac{1}{n} \sum_{k=n_{2}+1}^{n}\left|\log s_{k}(\mathbf{F}-\alpha \mathbf{I})\right|$ converges to zero in probability, i.e. condition (C2) is proved. 
Thus, the conditions (C0), (C1), (C2) have been checked for the matrices $\mathbf{F}_{\mathbf{X}}$. For the Gaussian matrices $\mathbf{F}_{\mathbf{Y}}$, the proof is the same. We may now apply Theorem 4.4 to conclude that the limiting eigenvalue distributions of the matrices $\mathbf{F}_{\mathbf{X}}$ and $\mathbf{F}_{\mathbf{Y}}$ are the same (if existent). Thus, it remains to compute the limit of the empirical distribution of the eigenvalues of the matrix $\mathbf{F}_{\mathbf{Y}}$.

From now on, let the matrices $\mathbf{F}_{t}:=\mathbf{F}_{t}(\mathbf{Y})$ be defined as in the proof of Theorem 8.13 . We shall use the asymptotic freeness of matrices

$$
\mathbf{V}_{t}=\left[\begin{array}{cc}
\mathbf{0} & \mathbf{F}_{t} \\
\mathbf{F}_{t}^{*} & \mathbf{0}
\end{array}\right] \text { and } \mathbf{J}(\alpha)=\left[\begin{array}{cc}
\mathbf{O} & -\alpha \mathbf{I} \\
-\bar{\alpha} \mathbf{I} & \mathbf{O}
\end{array}\right]
$$

As we have seen in the proof of Theorem 8.13, the limiting (mean) empirical spectral distribution of the matrices $\mathbf{F}_{t} \mathbf{F}_{t}^{*}$ is given by $\sigma \otimes \varrho_{t}$, with $S$-transform $S_{\sigma} \cdot S_{\varrho_{t}}$. Here, $\sigma$ and $\varrho_{t}$ denote the Marchenko-Pastur distribution and its induced measure under the mapping $x \mapsto(x+t)^{-1} x(x+t)^{-1}$, respectively. Thus, the limiting (mean) empirical spectral distribution of the matrices $\mathbf{V}_{t}$ is given by $\mathcal{Q}^{-1}\left(\sigma \otimes \varrho_{t}\right)$, where $\mathcal{Q}$ is as in (5.12), and the limiting spectral distribution of the matrices $\mathbf{V}_{t}(\alpha):=\mathbf{V}_{t}+\mathbf{J}(\alpha)$ is given by $\mathcal{Q}^{-1}\left(\sigma \otimes \varrho_{t}\right) \boxplus T(\alpha)$, where $T(\alpha)$ is as in Section 6. By a variant of Lemma 8.14 for shifted matrices as well as relations (5.9) and (5.10), it then follows that the limiting (mean) empirical spectral distributions of the matrices $\mathbf{V}$ and $\mathbf{V}(\alpha):=\mathbf{V}+\mathbf{J}(\alpha)$ are given by $\mathcal{Q}^{-1}(\sigma \otimes \varrho)$ and $\mathcal{Q}^{-1}(\sigma \otimes \varrho) \boxplus T(\alpha)$, respectively.

Moreover, the $S$-transform of the limiting eigenvalue distribution of $\mathbf{F F}^{*}$ is given by

$$
S_{\mathbf{F F}^{*}}(z)=-\frac{z}{z+1},
$$

as we have seen in the proof of Theorem 8.13, Thus, by (5.13), the $S$-transform of the limiting eigenvalue distribution of $\mathbf{V}$ is given by

$$
S_{\mathbf{V}}(z)=i \text {. }
$$

Finally, by Theorem 6.1, the Stieltjes transform $g_{t}(z, \alpha)$ associated with the matrices $\mathbf{V}_{t}(\alpha)$ satisfies the system of equations (6.16) with $S_{\mathbf{V}}$ replaced by $S_{\mathbf{V}_{t}}$. It therefore follows by continuity that the Stieltjes transform $g(z, \alpha)$ associated with the matrices $\mathbf{V}(\alpha)$ satisfies the system of equations (6.16).

Thus, the assumptions stated above Assumption 7.3 are satisfied, and we may apply Theorem 7.6. Solving now the system

$$
\begin{array}{r}
\psi(\alpha)(1-\psi(\alpha))=|\alpha|^{2} \varkappa^{2}(\alpha), \\
\varkappa(\alpha)=1-\psi(\alpha),
\end{array}
$$

we obtain

$$
\psi(\alpha)=\frac{|\alpha|^{2}}{1+|\alpha|^{2}}, \quad \varkappa(\alpha)=\frac{1}{1+|\alpha|^{2}}
$$

and

$$
u \frac{\partial \psi}{\partial u}+v \frac{\partial \psi}{\partial v}=\frac{2|\alpha|^{2}}{\left(1+|\alpha|^{2}\right)^{2}}
$$


The last equality and equality (7.10) together imply

$$
f(u, v)=\frac{1}{\pi\left(1+\left(u^{2}+v^{2}\right)\right)^{2}} .
$$

Thus Theorem 8.22 is proved.

\subsubsection{Product of Independent Matrices from Spherical Ensemble}

For fixed $m \geq 1$, let $\mathbf{X}^{(q)}, q=1, \ldots, 2 m$, be independent $n \times n$ random matrices with independent entries $\frac{1}{\sqrt{n}} X_{j k}^{(q)}$. Suppose that (8.1) or (8.2) holds. Consider the matrix $\mathbf{F}_{m}=$ $\mathbf{F}_{m}(\mathbf{X})=\prod_{q=1}^{m} \mathbf{X}^{(2 q-1)}\left(\mathbf{X}^{(2 q)}\right)^{-1}$, and denote by $\mu_{n}$ its empirical spectral distribution.

Theorem 8.24. Let the r.v.'s $X_{j k}^{(q)}$ for $q=1, \ldots, 2 m$ and $j, k=1, \ldots, n$ satisfy the condition (8.65). Then the measures $\mu_{n}$ weakly converge in probability to the measure $\mu$ with Lebesgue density

$$
p(x, y)=\frac{1}{\pi m\left(u^{2}+v^{2}\right)^{\frac{m-1}{m}}\left(1+\left(u^{2}+v^{2}\right)^{\frac{1}{m}}\right)^{2}} .
$$

Proof. The condition of Remark 4.5 follows from Theorem 8.15 . Conditions $(C 0)-(C 2)$ follow from induction principle and the proof of Theorem 8.22. Indeed, for $\alpha \in \mathbb{C}$, we have the representation

$$
\mathbf{F}_{m}-\alpha \mathbf{I}=\mathbf{F}_{m-1}\left(\mathbf{X}^{(2 m-1)}-\alpha \mathbf{F}_{m-1}^{-1} \mathbf{X}^{(2 m)}\right)\left(\mathbf{X}^{(2 m)}\right)^{-1}
$$

Write $s_{1}(\mathbf{A}) \geq \cdots \geq s_{n}(\mathbf{A})$ for the singular values of the matrix $\mathbf{A}$. Then, similarly as in 28], Theorem 3.3.14(c), we have, for any $k=1, \ldots, n$ and for any function $f$ such that $\varphi(t)=f\left(\mathrm{e}^{t}\right)$ is increasing and convex,

$$
\sum_{j=1}^{k} f\left(s_{j}\left(\mathbf{F}_{m}-\alpha \mathbf{I}\right)\right) \leq \sum_{j=1}^{k} f\left(s_{j}\left(\mathbf{F}_{m-1}\right) s_{j}\left(\mathbf{X}^{(2 m-1)}-\alpha \mathbf{F}_{m-1}^{-1} \mathbf{X}^{(2 m)}\right) s_{j}\left(\left(\mathbf{X}^{(2 m)}\right)^{-1}\right)\right) .
$$

Now use similar arguments as in the proof of Theorem 8.22. Thus, Theorem 4.4 and Remark 4.5 are applicable, and it remains to determine the limiting empirical spectral distribution in the Gaussian case.

Write $\mathbf{F}=\mathbf{F}(\mathbf{Y})$ for the products of independent "Gaussian" spherical matrices. To find their limiting empirical spectral distribution, we use the results from Section 7 For brevity, we give only a formal proof; it is straightforward (although a bit cumbersome) to make this proof rigorous by using similar arguments as in the proof of Theorem 8.22 (using a variant of the regularization lemma 8.17 this time). First, we find the $S$-transform $S_{\mathbf{F F}^{*}}(z)$ associated with the matrices $\mathbf{W}:=\mathbf{F F}^{*}$. Remember that, for any $q=1, \ldots, m$, the $S$-transform associated with the matrices $\mathbf{Y}^{(q)} \mathbf{Y}^{(q+1)^{-1}}\left(\mathbf{Y}^{(q+1)^{-1}}\right)^{*}\left(\mathbf{Y}^{(q)}\right)^{*}$ is given by

$$
-\frac{z}{z+1}
$$


by (8.75). Thus, by the multiplicative property of $S$-transform, we formally have

$$
S_{\mathbf{W}}(z)=\left(-\frac{z}{z+1}\right)^{m} .
$$

Using (5.13), it follows that

$$
S_{\mathbf{V}}(z)=i\left(-\frac{z}{z+1}\right)^{\frac{m-1}{2}} .
$$

Solving now the system

$$
\begin{array}{r}
\psi(1-\psi)=|\alpha|^{2} \varkappa^{2}, \\
\varkappa=(1-\psi)\left(\frac{1-\psi}{\psi}\right)^{\frac{m-1}{2}},
\end{array}
$$

we find that

$$
\psi=\frac{|\alpha|^{\frac{2}{m}}}{1+|\alpha|^{\frac{2}{m}}}
$$

and

$$
u \frac{\partial \psi}{\partial u}+v \frac{\partial \psi}{\partial v}=\frac{2|\alpha|^{\frac{2}{m}}}{m\left(1+|\alpha|^{\frac{2}{m}}\right)^{2}} .
$$

The last equality and equality (7.10) together imply

$$
f(u, v)=\frac{1}{\pi m\left(u^{2}+v^{2}\right)^{\frac{m-1}{m}}\left(1+\left(u^{2}+v^{2}\right)^{\frac{1}{m}}\right)^{2}} .
$$

Thus Theorem 8.24 is proved.

\section{A Appendix}

\section{A.1 Variance of Stieltjes Transforms}

In this subsection, $\mathbf{F}=\mathbf{F}_{\mathbf{X}}$ is defined as in Section 1, and $\mathbf{V}$ and $\mathbf{R}$ are the Hermitian matrices defined by

$$
\mathbf{V}=\left[\begin{array}{cc}
\mathbf{O} & \mathbf{F}+\mathbf{B} \\
(\mathbf{F}+\mathbf{B})^{*} & \mathbf{O}
\end{array}\right], \quad \mathbf{R}=(\mathbf{V}-z \mathbf{I})^{-1}
$$

where $\mathbf{B}$ is a non-random matrix and $z=u+i v$ with $v>0$.

Lemma A.1. Suppose that the rank condition (3.2) holds. Then

$$
\mathbf{E}\left|\frac{1}{n} \operatorname{Tr} \mathbf{R}-\mathbf{E} \frac{1}{n} \operatorname{Tr} \mathbf{R}\right|^{2} \leq \frac{C}{n v^{2}} .
$$


Proof. We introduce the $\sigma$-algebras $\mathfrak{N}_{q, j}=\sigma\left\{X_{l k}^{(q)}, j<l \leq n_{q-1}, k=1, \ldots, n_{q} ; X_{p k}^{(r)}\right.$, $\left.r=q+1, \ldots m, p=1, \ldots, n_{r-1}, k=1, \ldots, n_{r}\right\}$ and use the representation

$$
\operatorname{Tr} \mathbf{R}-\mathbf{E} \operatorname{Tr} \mathbf{R}=\sum_{q=1}^{m} \sum_{j=1}^{n_{q-1}}\left(\mathbf{E}_{q, j-1} \operatorname{Tr} \mathbf{R}-\mathbf{E}_{q, j} \operatorname{Tr} \mathbf{R}\right)
$$

where $\mathbf{E}_{q, j}$ denotes conditional expectation given the $\sigma$-algebra $\mathfrak{R}_{q, j}$. Note that $\mathfrak{R}_{q, n_{q-1}}=$ $\mathfrak{M}_{q+1,0}$. Furthermore, we introduce the matrices $\mathbf{X}^{(q, j)}$ obtained from $\mathbf{X}^{(q)}$ by replacing the entries $X_{j k}^{(q)}\left(k=1, \ldots, n_{q}\right)$ by zero's. Define the matrices

$$
\mathbf{F}^{(q, j)}=\mathbb{F}\left(\mathbf{X}^{(1)}, \ldots, \mathbf{X}^{(q-1)}, \mathbf{X}^{(q, j)}, \mathbf{X}^{(q+1)}, \ldots, \mathbf{X}^{(m)}\right)
$$

and

$$
\mathbf{V}^{(q, j)}=\left[\begin{array}{cc}
\mathbf{O} & \mathbf{F}^{(q, j)}+\mathbf{B} \\
\left(\mathbf{F}^{(q, j)}+\mathbf{B}\right)^{*} & \mathbf{O}
\end{array}\right], \quad \mathbf{R}^{(q, j)}=\left(\mathbf{V}^{(q, j)}-z \mathbf{I}\right)^{-1} .
$$

Note that $\mathbf{E}_{q, j} \operatorname{Tr} \mathbf{R}^{(q, j)}=\mathbf{E}_{q, j-1} \operatorname{Tr} \mathbf{R}^{(q, j)}$, and we may write

$$
\operatorname{Tr} \mathbf{R}-\mathbf{E} \operatorname{Tr} \mathbf{R}=\sum_{q=1}^{m} \sum_{j=1}^{n_{q-1}}\left(\mathbf{E}_{q, j-1}\left(\operatorname{Tr} \mathbf{R}-\operatorname{Tr} \mathbf{R}^{(q, j)}\right)-\mathbf{E}_{q, j}\left(\operatorname{Tr} \mathbf{R}-\operatorname{Tr} \mathbf{R}^{(q, j)}\right)\right),
$$

and

$$
\mathbf{E}|\operatorname{Tr} \mathbf{R}-\mathbf{E} \operatorname{Tr} \mathbf{R}|^{2}=\sum_{q=1}^{m} \sum_{j=1}^{n_{q-1}} \mathbf{E}\left|\mathbf{E}_{q, j-1}\left(\operatorname{Tr} \mathbf{R}-\operatorname{Tr} \mathbf{R}^{(q, j)}\right)-\mathbf{E}_{q, j}\left(\operatorname{Tr} \mathbf{R}-\operatorname{Tr} \mathbf{R}^{(q, j)}\right)\right|^{2} .
$$

By the rank inequality of Bai, we have

$$
\left|\operatorname{Tr} \mathbf{R}-\operatorname{Tr} \mathbf{R}^{(q, j)}\right| \leq \frac{\operatorname{rank}\left\{\mathbf{V}-\mathbf{V}^{(q, j)}\right\}}{v} .
$$

By the rank condition (3.2), we have

$$
\operatorname{rank}\left\{\mathbf{V}-\mathbf{V}^{(q, j)}\right\} \leq 2 \operatorname{rank}\left\{\mathbf{F}-\mathbf{F}^{(q, j)}\right\} \leq 2 C(\mathbb{F}) \operatorname{rank}\left\{\mathbf{X}^{(q)}-\mathbf{X}^{(q, j)}\right\} \leq 2 C(\mathbb{F}) .
$$

This concludes the proof of Lemma A.1.

\section{A.2 S-Transform for Rectangular Matrices}

The $S$-transform of a compactly supported probability distribution on $\mathbb{R}$ was introduced in Section 5. It is well-known that for free random variables $\xi, \eta \geq 0$, with $\xi, \eta \neq 0$,

$$
S_{\xi \eta}(z)=S_{\eta}(z) S_{\xi}(z)
$$

We may interpret this equality for random matrices as follows. For each $n \in \mathbb{N}$, let $\mathbf{X}_{n}$ and $\mathbf{Y}_{n}$ be two random square matrices of size $n \times n$. Assume that the matrices $\mathbf{X}_{n}^{*} \mathbf{X}_{n}$ 
and $\mathbf{Y}_{n} \mathbf{Y}_{n}^{*}$ are asymptotically free and that the mean empirical spectral distributions of the matrices $\mathbf{X}_{n} \mathbf{X}_{n}^{*}$ and $\mathbf{Y}_{n} \mathbf{Y}_{n}^{*}$ converge in moments to compactly supported probability measures $\mu$ and $\nu$, respectively, with $\mu, \nu \neq \delta_{0}$. Then the mean empirical spectral distributions of the matrices $\mathbf{X}_{n} \mathbf{Y}_{n} \mathbf{Y}_{n}^{*} \mathbf{X}_{n}^{*}$ converge in moments to the probability measure $\mu \otimes \nu$ and

$$
S_{\mu \bowtie \nu}(z)=S_{\mu}(z) S_{\nu}(z) .
$$

In the case of rectangular matrices this relation is not true anymore. The next lemma gives the correct relation for products of rectangular matrices.

Lemma A.2. For each $n \in \mathbb{N}$, let $\mathbf{X}_{n}$ and $\mathbf{Y}_{n}$ be two rectangular random matrices of the sizes $n \times p_{n}$ and $p_{n} \times \widetilde{p}_{n}$, respectively, and assume that $y=\lim _{n \rightarrow \infty} \frac{n}{p_{n}} \in(0, \infty)$. Assume additionally that the matrices $\mathbf{X}_{n}^{*} \mathbf{X}_{n}$ and $\mathbf{Y}_{n} \mathbf{Y}_{n}^{*}$ are asymptotically free and that the mean empirical spectral distributions of the matrices $\mathbf{X}_{n} \mathbf{X}_{n}^{*}$ and $\mathbf{Y}_{n} \mathbf{Y}_{n}^{*}$ converge in moments to compactly supported probability measures $\mu$ and $\nu$, respectively, with $\mu, \nu \neq \delta_{0}$. Denote by $S_{\mu}$ and $S_{\nu}$ the corresponding $S$-transforms. Then the mean empirical spectral distributions of the matrices $\mathbf{X}_{n} \mathbf{Y}_{n} \mathbf{Y}_{n}^{*} \mathbf{X}_{n}^{*}$ converge in moments to a probability measure $\xi$, and the corresponding $S$-transform $S_{\xi}$ is equal to

$$
S_{\xi}(z)=S_{\mu}(z) S_{\nu}(z y) .
$$

Proof. By slight abuse of notation, given a sequence $\left(\mathbf{A}_{n}\right)_{n \in \mathbb{N}}$ of self-adjoint matrices, we denote by $\mu_{\mathbf{A}}$ the limiting eigenvalue distribution and by $S_{\mathbf{A}}(z)$ the corresponding $S$-transform (if existent). For definiteness, assume that $n \leq p_{n}$. It is easy to see that

$$
\mu_{\mathbf{Y Y}} \mathbf{X}^{*} \mathbf{X}=y \mu_{\mathbf{X Y} \mathbf{Y}^{*} \mathbf{X}^{*}+(1-y) \delta_{0}}
$$

where $\delta_{0}$ denotes the unit atom at zero. From here it follows that

$$
S_{\mathbf{Y} \mathbf{Y}^{*} \mathbf{X}^{*} \mathbf{X}}(z)=\frac{z+1}{z+y} S_{\mathbf{X Y} \mathbf{Y}^{*} \mathbf{X}^{*}}\left(\frac{z}{y}\right) .
$$

We may rewrite this equality as follows

$$
S_{\mathbf{X Y Y}} \mathbf{X}^{*}(z)=\frac{y(z+1)}{y z+1} S_{\mathbf{Y Y}^{*} \mathbf{X}^{*} \mathbf{X}}(z y) .
$$

By asymptotic freeness and the multiplicative property of the $S$-transform, we have

$$
S_{\mathbf{Y} \mathbf{Y}^{*} \mathbf{X}} \mathbf{X}(z y)=S_{\mathbf{Y Y}^{*}}(z y) S_{\mathbf{X} * \mathbf{X}}(z y) .
$$

(In particular, the limiting eigenvalue distribution $\mu_{\mathbf{Y} \mathbf{Y}^{*} \mathbf{X}^{*} \mathbf{X}}$ exists.) By the same argument as for (A.2), we get

$$
S_{\mathbf{X X}^{*}}(z)=\frac{y(z+1)}{y z+1} S_{\mathbf{X}^{*} \mathbf{X}}(z y) .
$$

The three last equalities together imply the result of Lemma. 
Remark A.3. Using the preceding results, it is easy to see why the $m$ th power of a random square matrix $\mathbf{Y}_{n}$ and the product $\mathbf{Y}_{n}^{(1)} \cdots \mathbf{Y}_{n}^{(m)}$ of $m$ independent copies of this matrix should have the same limiting singular value and eigenvalue distributions. Indeed, let $\mathbf{Y}_{n}$ be bi-unitary invariant random square matrices such that the empirical spectral distribution of the matrices $\mathbf{Y}_{n} \mathbf{Y}_{n}^{*}$ converges weakly in probability as well as in moments to a compactly supported probability measure $\mu_{\mathbf{Y Y}^{*}}$.

Then, similarly as in Hiai and Petz [27, using the singular value decomposition of the matrix $\mathbf{Y}_{n}$, one can show that $\mathbf{Y}_{n} \mathbf{Y}_{n}^{*}$ and $\mathbf{Y}_{n}^{*} \mathbf{Y}_{n}$ are asymptotically free, and it follows from Equation (A.1) (and induction) that $\mathbf{Y}_{n}^{m}\left(\mathbf{Y}_{n}^{m}\right)^{*}$ converges in moments to $\mu_{\mathbf{Y} \mathbf{Y}^{*}}^{\otimes m}$. A similar argument, also based on Equation (A.1), shows that the same is true for $\left(\mathbf{Y}_{n}^{(1)} \cdots \mathbf{Y}_{n}^{(m)}\right)\left(\mathbf{Y}_{n}^{(1)} \cdots \mathbf{Y}_{n}^{(m)}\right)^{*}$, where $\mathbf{Y}_{n}^{(1)}, \ldots, \mathbf{Y}_{n}^{(m)}$ are $m$ independent copies of $m$. Thus, the matrices $\mathbf{Y}_{n}^{m}$ and $\mathbf{Y}_{n}^{(1)} \cdots \mathbf{Y}_{n}^{(m)}$ will have the same limiting singular value distributions.

Now the $S$-transform of the limiting singular value distribution of the shifted matrices $\mathbf{F}-\alpha \mathbf{I}$ is well defined by $\alpha$ and the limiting singular value distribution of the matrix $\mathbf{F}$. To prove this we must use the additive property of the $R$-transform and the correspondence between $R$-and $S$-transforms. Furthermore, note that the limit measure for the eigenvalue distribution is well defined by its logarithmic potential and that we may reconstruct the logarithmic potential from the family of the singular value distribution of the shifted matrices. It therefore follows that the limiting eigenvalue distribution of the matrices $\mathbf{Y}_{n}^{m}$ and $\mathbf{Y}_{n}^{(1)} \cdots \mathbf{Y}_{n}^{(m)}$ will also be the same.

But the eigenvalues of the $m$ th power of a matrix are the $m$ th powers of the eigenvalues of that matrix. For example, if the limiting eigenvalue distribution of the random matrix $\mathbf{Y}_{n}$ is the circular law, then the limiting eigenvalue distribution of the product $\mathbf{Y}_{n}^{(1)} \cdots \mathbf{Y}_{n}^{(m)}$ is the $m$ th power of the uniform distribution in the unit disc.

\section{A.3 Bounds on Singular Values}

Throughout this subsection, let $\mathbf{X}$ denote an $n \times n$ random matrix with independent entries $\frac{1}{\sqrt{n}} X_{j k}$ such that $\mathbf{E} X_{j k}=0$ and $\mathbf{E}\left|X_{j k}\right|^{2}=1$. Let $s_{1}(\mathbf{X}) \geq \cdots \geq s_{n}(\mathbf{X})$ denote the singular values of the matrix $\mathbf{X}$. Then we have the following result:

Lemma A.4. We have $\lim _{t \rightarrow \infty} \limsup _{n \rightarrow \infty} \operatorname{Pr}\left\{\frac{1}{n} \sum_{k=1}^{n} s_{k}^{2}(\mathbf{X}) \geq t\right\}=0$.

Proof. This follows from the observation that

$$
\mathbf{E}\left(\frac{1}{n} \sum_{k=1}^{n} s_{k}^{2}(\mathbf{X})\right)=\frac{1}{n} \mathbf{E}\|\mathbf{X}\|_{2}^{2}=\frac{1}{n^{2}} \sum_{j, k} \mathbf{E}\left|X_{j k}\right|^{2}=1
$$

and Markov's inequality.

Henceforward, assume additionally that the r.v.'s $X_{j k}$ satisfy the condition

$$
\max _{j, k} \mathbf{E}\left|X_{j k}\right|^{2} \mathbb{I}\left\{\left|X_{j k}\right|>M\right\} \rightarrow 0, \quad \text { as } \quad M \rightarrow \infty .
$$


Under these assumptions, we have the following bounds on the small singular values, see Götze and Tikhomirov, 24], Theorem 4.1 and [25], Lemma 5.2 and Proposition 5.1. (For the i.i.d. case similar results were obtained by Tao and $\mathrm{Vu}$, 41, Lemma 4.1 and 4.2.) Let $s_{1}(\mathbf{X}-\alpha \mathbf{I}) \geq \cdots \geq s_{n}(\mathbf{X}-\alpha \mathbf{I})$ denote the singular values of the matrix $\mathbf{X}-\alpha \mathbf{I}$.

Lemma A.5. Suppose that condition (A.3) holds. Then, for any fixed $\alpha \in \mathbb{C}$, there exist positive constants $Q$ and $B$ such that

$$
\operatorname{Pr}\left\{s_{n}(\mathbf{X}-\alpha \mathbf{I}) \leq n^{-Q}\right\} \leq n^{-B} .
$$

For a proof of this lemma see the proof of Theorem 4.1 in [24].

Lemma A.6. Suppose that condition (A.3) holds. Then, for any fixed $\alpha \in \mathbb{C}$, there exists a constant $0<\gamma<1$ such that for any sequence $\delta_{n} \rightarrow 0$,

$$
\lim _{n \rightarrow \infty} \frac{1}{n} \sum_{n_{1} \leq j \leq n_{2}} \ln s_{j}(\mathbf{X}-\alpha \mathbf{I})=0 \quad \text { almost surely }
$$

with $n_{1}=\left[n-n \delta_{n}\right]+1$ and $n_{2}=\left[n-n^{\gamma}\right]$.

For a proof of this lemma see the proof of inequality (5.17) and Lemma 5.2 in [25].

For the investigation of the spherical ensembles, we need the following extensions of these results; see Equations (5.9) and (5.17) in [25].

Lemma A.7. Suppose that condition (A.3) holds. Then, for any $K>0$ and $L>0$, there exist positive constants $Q$ and $B$ such that for any non-random matrix $\mathbf{M}$ with $\|\mathbf{M}\|_{2} \leq K n^{L}$, we have

$$
\operatorname{Pr}\left\{s_{n}(\mathbf{X}-\mathbf{M}) \leq n^{-Q}\right\} \leq n^{-B} .
$$

Lemma A.8. Suppose that condition (A.3) holds. Then, for any fixed $K>0$ and $L>0$, there exist constants $0<\gamma<1$ and $c>0$ such that for any non-random matrix $\mathbf{M}$ with $\|\mathbf{M}\|_{2} \leq K n^{L}$, we have

$$
\operatorname{Pr}\left\{s_{j}(\mathbf{X}-\mathbf{M}) \geq c \frac{n-j}{n} \quad \text { for all } j=1, \ldots, n-n^{\gamma}\right\} \geq 1-\exp \left(-n^{\gamma}\right) .
$$

\section{A.4 Technical Details for Section 7}

In this subsection, we state some technical lemmas which have been used in Section 7.

Using similar arguments as in the proof of Theorem 4.4 / Remark 4.5, we may prove the following.

Lemma A.9. Assume that the matrices $\mathbf{F}_{\mathbf{Y}}$ satisfy the conditions $(C 0),(C 1)$ and $(C 2)$. Moreover, assume that the singular value distributions of the matrices $\mathbf{F}_{\mathbf{Y}}$ converge weakly 
in probability to a non-random probability measure $\nu$. Then the logarithm is integrable w.r.t. $\nu$, and we have

$$
\lim _{n \rightarrow \infty}\left(\frac{1}{n} \sum_{k=1}^{n} \log s_{k}\left(\mathbf{F}_{\mathbf{Y}}\right)\right) \rightarrow \int_{0}^{\infty} \log (x) d \nu(x) \quad \text { in probability }
$$

as well as

$$
\lim _{n \rightarrow \infty}\left(\frac{1}{n} \sum_{k=1}^{n}\left|\log s_{k}\left(\mathbf{F}_{\mathbf{Y}}\right)\right|\right) \rightarrow \int_{0}^{\infty}|\log (x)| d \nu(x) \quad \text { in probability. }
$$

Proof. Clearly, the main problem is to show that the logarithm is integrable w.r.t. $\nu$. Once this is shown, it is straightforward to adapt the proof of Theorem 4.4, replacing the singular value distributions of the matrices $\mathbf{F}_{\mathbf{Y}}$ with the fixed distribution $\nu$. We will show separately that $\log ^{+}:=\max \{+\log , 0\}$ and $\log ^{-}:=\max \{-\log , 0\}$ are integrable w.r.t. $\nu$. In doing so, we write $\nu_{n}$ for the singular value distribution of $\mathbf{F}_{\mathbf{Y}}$.

Let us begin with the positive part. First of all, passing to a suitable subsequence, we may assume w.l.o.g. that $\nu_{n} \Rightarrow \nu$ almost surely. Now, by assumption (C0), there exists a constant $K>0$ such that

$$
\operatorname{Pr}\left\{\int x^{p} d \nu_{n} \geq K\right\} \leq \frac{1}{2}
$$

for all sufficiently large $n \in \mathbb{N}$. This implies that the event

$$
A:=\left\{\omega: \int x^{p} \nu_{n}(\omega, d x) \leq K \text { infinitely often }\right\}
$$

has positive probability. Fix $\omega \in \Omega$ such that $\nu_{n}(\omega) \Rightarrow \nu$ and a subsequence $\left(\nu_{n_{k}}(\omega)\right)$ such that $\int x^{p} \nu_{n_{k}}(\omega, d x) \leq K$ for all $k \in \mathbb{N}$. Then $x^{p / 2}$, and hence $\log ^{+}(x)$, is uniformly integrable w.r.t. $\left(\nu_{n_{k}}(\omega)\right)$, and it follows that $\log ^{+} \in L^{1}(\nu)$.

Let us now consider the negative part. Again, we may select a subsequence $\left(\nu_{n_{k}}\right)$ such that $\nu_{n_{k}} \Rightarrow \nu$ almost surely. Moreover, using monotone convergence and weak convergence, we have

$$
\begin{aligned}
\int \log ^{-} d \nu= & \lim _{a \rightarrow \infty} \int\left(\log ^{-} \wedge a\right) d \nu \\
& =\lim _{a \rightarrow \infty} \lim _{k \rightarrow \infty} \int\left(\log ^{-} \wedge a\right) d \nu_{n_{k}} \leq \liminf _{k \rightarrow \infty} \int \log ^{-} d \nu_{n_{k}} \quad \text { almost surely. }
\end{aligned}
$$

Suppose by way of contradiction that $\int \log ^{-} d \nu=\infty$. Then, for any $l \in \mathbb{N}$, we have

$$
\lim _{k \rightarrow \infty} \int_{0}^{1 / l} \log ^{-} d \nu_{n_{k}}=\infty \quad \text { almost surely. }
$$

Thus, for any $l \in \mathbb{N}$, we may find an index $k(l)$ such that

$$
\int_{0}^{1 / l} \log ^{-} d \nu_{n_{k(l)}} \geq l \quad \text { with probability } \geq 1-2^{-l} .
$$


We may assume w.l.o.g. that the sequence $(k(l))$ is increasing. Thus, we obtain a subsequence (which we again denote by $\nu_{n_{k}}$, by abuse of notation) such that

$$
\int_{0}^{1 / k} \log ^{-} d \nu_{n_{k}} \geq k
$$

for almost all $k \in \mathbb{N}$. We now proceed similarly as in the proof of Theorem 4.4. Put $\delta_{k}:=1 / \log k, n_{1}(k):=\left[n_{k}-\delta_{k} n_{k}\right]+1$ and $n_{2}(k):=\left[n_{k}-n_{k}^{\gamma}\right]$. (We write $n_{1}(k)$ and $n_{2}(k)$ here to emphasize the dependence on $k$.) Then, by the same arguments as in the proof of Theorem 4.4, the probability of the following events tends to zero:

$$
\left\{s_{n_{k}}\left(\mathbf{F}_{\mathbf{Y}}\right)<n_{k}^{-Q}\right\},\left\{\frac{1}{n_{k}} \sum_{n_{1}(k) \leq j \leq n_{2}(k)}\left|\log s_{j}\left(\mathbf{F}_{\mathbf{Y}}\right)\right|>1\right\},\left\{s_{n_{1}(k)}\left(\mathbf{F}_{\mathbf{Y}}\right) \leq \frac{1}{k}\right\} .
$$

Thus, we may select a sequence $(k(l))$ such that with probability 1 , we have

$$
s_{n_{k(l)}}\left(\mathbf{F}_{\mathbf{Y}}\right) \geq n_{k(l)}^{-Q}, \frac{1}{n_{k(l)}} \sum_{n_{1}(k(l)) \leq j \leq n_{2}(k(l))}\left|\log s_{j}\left(\mathbf{F}_{\mathbf{Y}}\right)\right| \leq 1, s_{n_{1}(k(l))}\left(\mathbf{F}_{\mathbf{Y}}\right)>\frac{1}{k(l)}
$$

for almost all $l \in \mathbb{N}$. It then follows using (A.4) that with probability 1 , we have

$$
\begin{aligned}
k(l) \leq \int_{0}^{1 / k(l)} \log ^{-} d \nu_{n_{k(l)}} & \leq \frac{1}{n_{k(l)}} \sum_{j=n_{1}(k(l))}^{n_{2}(k(l))-1}\left|\log s_{j}\left(\mathbf{F}_{\mathbf{Y}}\right)\right| \\
& +\frac{1}{n_{k(l)}} \sum_{j=n_{2}(k(l))}^{n_{k(l)}}\left|\log s_{j}\left(\mathbf{F}_{\mathbf{Y}}\right)\right| \leq 1+\frac{1}{n_{k(l)}}\left(n_{k(l)}^{\gamma}+1\right) Q \log n_{k(l)}
\end{aligned}
$$

for almost all $l \in \mathbb{N}$. But this is a contradiction. We therefore come to the conclusion that $\log ^{-} \in L^{1}(\nu)$.

We now prove Lemma 7.4. For convenience, we repeat the statement of the lemma.

Lemma A.10 (= Lemma (7.4). Assumptions 7.2 and 7.3 hold for probability measures $\mu_{\mathbf{V}}$ such that $\mu_{\mathbf{V}}\left([-x,+x]^{c}\right)=\mathcal{O}\left(x^{-\eta}\right)(x \rightarrow \infty)$ for some $\eta>0$.

Proof. The proof consists of several parts. We will use the fact that the free additive convolution is monotone with respect to stochastic order $\leq_{\text {st }}$ (see e.g. Proposition 4.16 in Bercovici and Voiculescu [11]), i.e. we have

$$
\mu_{1} \leq_{\text {st }} \mu_{2} \wedge \nu_{1} \leq_{\text {st }} \nu_{2} \quad \Rightarrow \quad \mu_{1} \boxplus \mu_{2} \leq_{\text {st }} \nu_{1} \boxplus \nu_{2} .
$$


Preliminary Estimates. It follows from th.5 that $\mu_{\mathbf{V}}\left([-x,+x]^{c}\right)=\mathcal{O}\left(x^{-\eta}\right)(x \rightarrow \infty)$ implies $\mu_{\mathbf{V}(\alpha)}\left([-x,+x]^{c}\right)=\mathcal{O}\left(x^{-\eta}\right)(x \rightarrow \infty)$, where the $\mathcal{O}$-bound is locally uniform in $\alpha$. Thus, using integration by parts, we find that for any continuously differentiable (possibly complex-valued) function $f$ such that $f^{\prime}(x)=\mathcal{O}\left(|x|^{-1}\right)$ as $|x| \rightarrow \infty$, we have

$$
\int_{\mathbb{R}} f(x) d \mu_{\mathbf{V}(\alpha)}(x)=f(0)+\int_{0}^{\infty} f^{\prime}(y)\left(1-\mathcal{F}_{\mathbf{V}(\alpha)}(y)\right) d y-\int_{-\infty}^{0} f^{\prime}(y) \mathcal{F}_{\mathbf{V}(\alpha)}(y) d y,
$$

where $\mathcal{F}_{\mathbf{V}(\alpha)}$ denotes the distribution function of $\mu_{\mathbf{V}(\alpha)}$. Therefore, for any $\alpha, \beta \in \mathbb{R}^{2}$,

$$
\int_{\mathbb{R}} f(x) d \mu_{\mathbf{V}(\alpha)}(x)-\int_{\mathbb{R}} f(x) d \mu_{\mathbf{V}(\beta)}(x)=\int_{\mathbb{R}} f^{\prime}(y)\left(\mathcal{F}_{\mathbf{V}(\beta)}(y)-\mathcal{F}_{\mathbf{V}(\alpha)}(y)\right) d y .
$$

Suppose w.l.o.g. that $|\alpha| \leq|\beta|$, and set $m:=\frac{|\alpha|+|\beta|}{2}, \varepsilon:=|\beta|-|\alpha|$ and $\xi:=\mu_{\mathbf{V}} \boxplus T(m)$. Then, by (A.5), we have

$$
\xi \boxplus \delta_{-\varepsilon / 2}=\mu_{\mathbf{V}} \boxplus \frac{1}{2}\left(\delta_{-|\beta|}+\delta_{+|\alpha|}\right) \leq_{\text {st }} \mu_{\mathbf{V}(\alpha)}, \mu_{\mathbf{V}(\beta)} \leq_{\text {st }} \mu_{\mathbf{V}} \boxplus \frac{1}{2}\left(\delta_{-|\alpha|}+\delta_{+|\beta|}\right)=\xi \boxplus \delta_{+\varepsilon / 2}
$$

and therefore

$$
\left|\mathcal{F}_{\mathbf{V}(\beta)}(x)-\mathcal{F}_{\mathbf{V}(\alpha)}(x)\right| \leq \mathcal{F}_{\xi}\left(x+\frac{1}{2} \varepsilon\right)-\mathcal{F}_{\xi}\left(x-\frac{1}{2} \varepsilon\right)
$$

It follows that

$$
\left|\int_{\mathbb{R}} f^{\prime}(y)\left(\mathcal{F}_{\mathbf{V}(\beta)}(y)-\mathcal{F}_{\mathbf{V}(\alpha)}(y)\right) d y\right| \leq\left\|f^{\prime}\right\|_{\infty} \int_{\mathbb{R}}\left(\mathcal{F}_{\xi}\left(y+\frac{1}{2} \varepsilon\right)-\mathcal{F}_{\xi}\left(y-\frac{1}{2} \varepsilon\right)\right) d y=\varepsilon .
$$

Combining these inequalities, we find that for any $\alpha, \beta \in \mathbb{R}^{2}$ and for any function $f$ with the above-mentioned properties, we have

$$
\left|\int_{\mathbb{R}} f(x) d \mu_{\mathbf{V}(\alpha)}(x)-\int_{\mathbb{R}} f(x) d \mu_{\mathbf{V}(\beta)}(x)\right| \leq|\alpha-\beta|\left\|f^{\prime}\right\|_{\infty} .
$$

In particular, the integral $\int f(x) d \mu_{\mathbf{V}(\alpha)}(x)$ is continuous in $\alpha$.

Proof of Continuity. By general properties of the Stieltjes transform, the function $g(i y, \alpha)$ is locally uniformly continuous in $y$, uniformly in $\alpha$. Thus, it remains to show that the function $g(i y, \alpha)$ is continuous in $\alpha$. This follows by taking $f(x):=\frac{1}{x-i y}$ in (A.6), with $y>0$ fixed.

Proof of Differentiability. By general properties of the Stieltjes transform, the function $g(i y, \alpha)$ is differentiable with respect to $y$, with derivative

$$
\frac{\partial g}{\partial y}(i y, \alpha)=\int \frac{i}{(x-i y)^{2}} d \mu_{\mathbf{V}(\alpha)}(x) .
$$

It therefore follows by the same arguments as in the preceding paragraph that $\frac{\partial g}{\partial y}(i y, \alpha)$ is continuous.

The argument for $\frac{\partial g}{\partial u}(i y, \alpha)$ is a bit longer, and we confine ourselves to a rough sketch. It is straightforward to see (e.g. by using the additivity of the Voiculescu transform, 
see e.g. Corollary 5.7 in [1] ) that the Stieltjes transform $g(z, \alpha)$ is locally analytic in $(z, \alpha)$ around the point $\left(z_{0}, \alpha_{0}\right)$, for $\alpha_{0} \neq 0$ fixed and $z_{0} \in \mathbb{C}^{+}$with $\operatorname{Im} z_{0}$ sufficiently large. Thus, we locally have the power series expansions

$$
\begin{gathered}
g(z, \alpha)=\sum_{k=0}^{\infty} \sum_{j=0}^{\infty} c_{j k}\left(\alpha-\alpha_{0}\right)^{j}\left(z-z_{0}\right)^{k}=: \sum_{k=0}^{\infty} c_{k}(\alpha)\left(z-z_{0}\right)^{k}, \\
\frac{\partial g}{\partial u}(z, \alpha)=\sum_{k=0}^{\infty} \sum_{j=0}^{\infty} c_{j k} j\left(\alpha-\alpha_{0}\right)^{j-1}\left(z-z_{0}\right)^{k}=: \sum_{k=0}^{\infty} \widetilde{c}_{k}(\alpha)\left(z-z_{0}\right)^{k} .
\end{gathered}
$$

Suppose that the bivariate power series converge on the set of all $(z, \alpha)$ with $\left|z-z_{0}\right|<\varepsilon$ and $\left|\alpha-\alpha_{0}\right|<\varepsilon$, where $\varepsilon=\varepsilon\left(z_{0}, \alpha_{0}\right)>0$.

Let us investigate the growth of the coefficients, and hence the radius of convergence, of the univariate power series in $z$. Since

$$
\begin{gathered}
\left|c_{k}(\alpha)\right|=\left|\frac{1}{k !} \frac{\partial^{k}}{\partial z^{k}} g\left(z_{0}, \alpha\right)\right|=\left|\frac{1}{k !} \int \frac{k !}{(t-z)^{k+1}} d \mu_{\mathbf{V}(\alpha)}(d t)\right| \leq \frac{1}{\left(\operatorname{Im} z_{0}\right)^{k+1}}, \\
\left|\widetilde{c}_{k}(\alpha)\right|=\left|\frac{1}{k !} \frac{\partial^{k}}{\partial z^{k}} \frac{\partial}{\partial u} g\left(z_{0}, \alpha\right)\right|=\left|\frac{1}{k !} \frac{\partial}{\partial u} \frac{\partial^{k}}{\partial z^{k}} g\left(z_{0}, \alpha\right)\right| \leq \frac{k+1}{\left(\operatorname{Im} z_{0}\right)^{k+1}},
\end{gathered}
$$

where the last estimate follows from Equation (A.6), the two power series have radius of convergence $\geq \operatorname{Im} z_{0}$. Furthermore, since the functions $c_{k}(\alpha)$ and $\widetilde{c}_{k}(\alpha)$ are continuous in $\alpha$ and, for any fixed $\delta>0$, the two power series converge uniformly for $\left|z-z_{0}\right|<\left(\operatorname{Im} z_{0}\right)-\delta$ and $\left|\alpha-\alpha_{0}\right|<\varepsilon-\delta$, they represent continuous functions $f$ and $\widetilde{f}$ defined on the set $B\left(z_{0}, \operatorname{Im} z_{0}\right) \times B\left(\alpha_{0}, \varepsilon\right)$. (Here, $B(z, r)$ denotes the open ball of radius $r$ around the point $z$.) Thus, again by uniform convergence, we may conclude that the function $f$ is continuously differentiable with respect to $u$. Since the function $f(z, \alpha)$ coincides with $g(z, \alpha)$ on the set $B\left(z_{0}, \varepsilon\right) \times B\left(\alpha_{0}, \varepsilon\right)$ (by construction) and therefore on the set $B\left(z_{0}, \operatorname{Im} z_{0}\right) \times B\left(\alpha_{0}, \varepsilon\right)$ (by analytic continuation in $z$ ), this proves our claim about the existence and the continuity of $\frac{\partial g}{\partial u}(i y, \alpha)$.

Proof of (7.4). Since for fixed $\alpha, g(z, \alpha)$ is a non-constant analytic function in a certain open set containing the upper imaginary half-axis, there exists an at most countable set $Y_{\alpha}=\left\{y_{1}, y_{2}, y_{3}, \ldots\right\}$ such that for all $y \notin Y_{\alpha}$,

$$
g(i y, \alpha) \neq \frac{i}{2|\alpha|} \quad \text { and } \quad \frac{\partial g}{\partial y}(i y, \alpha) \neq 0 .
$$

For $y \notin Y_{\alpha}$, differentiating the second equation in (6.8) with respect to $y$, we get

$$
\begin{aligned}
-\frac{\partial g}{\partial y}(i y, \alpha)=\widetilde{S}_{\mathbf{V}}^{\prime}(-(1+ & \left.\left.i y g(i y, \alpha)-\frac{1}{2}+\frac{1}{2} \sqrt{1+4|\alpha|^{2} g(i y, \alpha)^{2}}\right)\right) \\
\times & {\left[-i g(i y, \alpha)-i y \frac{\partial g}{\partial y}(i y, \alpha)-\frac{2|\alpha|^{2} g(i y, \alpha) \frac{\partial g}{\partial y}(i y, \alpha)}{\sqrt{1+4|\alpha|^{2} g(i y, \alpha)^{2}}}\right], }
\end{aligned}
$$

where $\widetilde{S}_{\mathbf{V}}(z):=z S_{\mathbf{V}}(z)$. 
Now fix $\left(i y_{0}, \alpha_{0}\right)$ with $\alpha_{0} \neq 0$ and $y_{0} \notin Y_{\alpha_{0}}$. Then there exists a small neighborhood $N$ such that for $(i y, \alpha) \in N$, we have (A.7), (A.8), and

$$
F(g(i y, \alpha), i y, \alpha)=0
$$

where

$$
F(\zeta, i y, \alpha):=\zeta+\widetilde{S}_{\mathbf{V}}\left(-\left(1+i y \zeta-\frac{1}{2}+\frac{1}{2} \sqrt{1+4|\alpha|^{2} \zeta^{2}}\right)\right)
$$

and the sign of the square-root is constant in $N$. Note that $F$ is an analytic function with

$$
\begin{aligned}
\frac{\partial F}{\partial \zeta}(g(i y, \alpha), i y, \alpha)=1+\widetilde{S}_{\mathbf{V}}^{\prime}(-(1+i y g(i y, \alpha) & \left.\left.-\frac{1}{2}+\frac{1}{2} \sqrt{1+4|\alpha|^{2} g(i y, \alpha)^{2}}\right)\right) \\
& \times\left[-i y-\frac{2|\alpha|^{2} g(i y, \alpha)}{\sqrt{1+4|\alpha|^{2} g(i y, \alpha)^{2}}}\right] .
\end{aligned}
$$

Moreover, comparing (A.8) and (A.9), we see that

$$
\frac{\partial F}{\partial \zeta}(g(i y, \alpha), i y, \alpha)=\frac{i g(i y, \alpha)}{\frac{\partial g}{\partial y}(i y, \alpha)} \widetilde{S}_{\mathbf{V}}^{\prime}\left(-\left(1+i y g(i y, \alpha)-\frac{1}{2}+\frac{1}{2} \sqrt{1+4|\alpha|^{2} g(i y, \alpha)^{2}}\right)\right) \neq 0 .
$$

It therefore follows from the implicit function theorem for real-analytic functions that there exists a small neighborhood $\widetilde{N} \subset N$ of the point $\left(i y_{0}, \alpha_{0}\right)$ such that $g(i y, \alpha)$, the solution to the equation $F(\zeta, i y, \alpha)=0$, is analytic on $\widetilde{N}$, with gradient

$$
\frac{\partial g}{\partial(y, u, v)}=-\left(\frac{\partial F}{\partial \zeta}\right)^{-1} \frac{\partial F}{\partial(y, u, v)} .
$$

Equation (7.4) now follows by a straightforward calculation.

Existence of continuous extension. This follows from Lemma 6.4 and the subsequent Remark 6.5.

Proof of Assumption 7.3, Let $K$ be a compact set as in Assumption 7.3, and let $\alpha, \beta \in K$. For fixed $C>0$, consider the function $f(y):=\log \left(1+y^{2} / C^{2}\right)$. Since $f^{\prime}(y)=$ $\frac{2 y}{C^{2}+y^{2}}$, this function satisfies the conditions of Equation (A.6), and we obtain

$$
\left|\int \log \left(1+y^{2} / C^{2}\right) d \mu_{\mathbf{V}(\alpha)}(y)-\int \log \left(1+y^{2} / C^{2}\right) d \mu_{\mathbf{V}(\beta)}(y)\right| \leq|\alpha-\beta| / C,
$$

from which Assumption 7.3 follows immediately.

Acknowledgement. We thank Peter J. Forrester for pointing out some relevant references. 


\section{References}

[1] Akemann, G.; Ipsen, J.; Kieburg, M. Products of rectangular random matrices: Singular values and progressive scattering. Phys. Rev. E, vol. 88, 2013, 052118.

[2] Alexeev, N.; Götze, F.; Tikhomirov, A. N. Asymptotic distribution of singular values of powers of random matrices, Lithuanian math. J., vol. 50, no. 2, 2010, 121-132.

[3] Alexeev, N.; Götze, F.; Tikhomirov, A. N. On the singular spectrum of powers and products of random matrices, Doklady Mathematics, vol. 82, no. 1, 2010, 505-507.

[4] Alexeev, N.; Götze, F.; Tikhomirov, A. N. On the asymptotic distribution of singular values of products of large rectangular random matrices. Preprint, arXiv:1012.2586

[5] Alexeev, N.; Götze, F.; Tikhomirov, A. N. On the asymptotic distribution of the singular values of powers of random matrices. (Russian) Zapiski Nauchn. Seminarov POMI, vol. 408, 2012, 9-43.

[6] Arizmendi, O. E., Pérez-Abreu, V. The S-transform of symmetric probability measures with unbounded supports. Communicatión del CIMAT, No 1-08-20/19-112008 (PE-CIMAT).

[7] Bai, Z. D. Circular law. Ann. Probab. 25, no. 1, 1997, 494-529.

[8] Bai, Z.; Silverstein, J. W. Spectral analysis of large dimensional random matrices. Second edition. Springer Series in Statistics. Springer, New York, 2010. xvi+551 pp.

[9] Belinschi, S. T., Mai, T., Speicher, R. Analytic subordination theory of operatorvalued free additive convolution and the solution of a general random matrix problem. Preprint, arXiv:1303.3196.

[10] Bentkus, V. A new approach to approximations in probability theory and operator theory. (Russian) Liet. Mat. Rink. 43, no. 4, 2003, 444-470; translation in Lithuanian Math. J. 43, no. 4, 2003, 367-388.

[11] Bercovici, H., Voiculescu, D. Free convolution of measures with unbounded support. Indiana Univ. Math. J. 42, no. 3, 1993, 733-773.

[12] Biane, P. Processes with free increments. Math. Z. 227, 1998, 143-174.

[13] Bordenave, Ch. On the spectrum of sum and product of non-Hermitian random matrices. Electron. Commun. Probab. 16, 2011, 104-113.

[14] Bordenave, Ch., Chafaï, D. Around the circular law. Probab. Surv. 9, 2012, 1-89.

[15] Burda, Z., Janik, R. A., Waclaw, B. Spectrum of the product of independent random Gaussian matrices. Phys. Rev. E, vol. 81, 2010, 041132.

[16] Burda, Z., Jarosz, A., Livan, G., Nowak, M. A., Swiech, A. Eigenvalues and singular values of products of rectangular Gaussian random matrices. Phys. Rev. E, vol. 82, 2010, 061114. 
[17] Chatterjee, S. A generalization of the Lindeberg principle. Ann. Probab. 34, no. 6, 2006, 2061-2076.

[18] Chistyakov, G., Götze, F. The arithmetic of distributions in free probability theory. Cent. Eur. J. Math. 9, no. 5, 2011, 997-1050.

[19] Dykema, K. J., Nica, A., Voiculescu, D. Free random variables. A noncommutative probability approach to free products with applications to random matrices, operator algebras and harmonic analysis on free groups. CRM Monograph Series, 1. American Mathematical Society, Providence, RI, 1992. vi+70 pp.

[20] Forrester, P. J. Eigenvalue statistics for product complex Wishart matrices. J. Phys. A: Math. Theor. 47, 2014, 345202. arXiv:1401.2572.

[21] Forrester, P. J., Liu, D.-Z. Raney distributions and random matrix theory. Preprint, arXiv:1404.5759.

[22] Girko, V. L. The circular law. (Russian) Teor. Veroyatnost. i Primenen. 29, no. 4, 1984, 669-679.

[23] Götze, F.; Tikhomirov, A. N. Rate of convergence in probability to the MarchenkoPastur law. Bernoulli 10, no. 3, 2004, 503-548.

[24] Götze, F.; Tikhomirov, A. N. The circular law for random matrices. Ann. Prob. 38 , no. 4, 2010, 1444-1491.

[25] Götze, F.; Tikhomirov, A. N. On the asymptotic spectrum of products of independent random matrices. Preprint, arXiv:1012.2710.

[26] Götze, F.; Tikhomirov, A. N. Limit theorems for products of large random matrices. Talk given at the conference "Random matrices and their applications" in Paris in October 2012, available at http://congres-math.univ-mlv.fr/ sites/congres-math.univ-mlv.fr/files/Tikhomirov.pdf

[27] Hiai, F.; Petz D. Asymptotic Freeness Almost Everywhere for Random Matrices. Acta. Sci. Math. (Szeged), 66, 2000, 801-826.

[28] Horn, R.; Johnson, Ch. Topics in Matrix analysis. Cambridge University Press, 1991, 607 pp.

[29] Kuijlaars, A.; Zhang, L. Singular values of products of Ginibre random matrices, multiple orthogonal polynomials and hard edge scaling limits. Preprint, arXiv:1308.1003.

[30] Lytova, A.; Pastur, L. Central limit theorem for linear eigenvalue statistics of random matrices with independent entries. Ann. Probab. 37, no. 5, 2009, 17781840.

[31] Marchenko, V.; Pastur, L. The eigenvalue distribution in some ensembles of random matrices. Math. USSR Sbornik, no. 1, 1967, 457-483.

[32] Mays, A. A real quaternion spherical ensemble of random matrices. Preprint, arXiv:1209.0888. 
[33] Müller, R. On the asymptotic eigenvalue distribution of concatenated vector-valued fading channels. IEEE Trans. Inf. Theory 48, no. 7, 2002, 2086-2091.

[34] Nica, A. R-Transforms in Free Probability. Lectures in special semester "Free probability theory and operator spaces." IHP, Paris 1999. Available at http: //www . math. uwaterloo.ca/ anica/NOTES/section11.pdf

[35] Pan, G.; Zhou, W. Circular law, extreme singular values and potential theory. J. Multivariate Anal. 101, no. 3, 2010, 645-656.

[36] Pastur, L.; Shcherbina, M. Eigenvalue distribution of large random matrices. Mathematical Surveys and Monographs, 171. American Mathematical Society, Providence, RI, 2011. xiv+632 pp.

[37] Rao, N. R.; Speicher, R. Multiplication of free random variables and the S-transform: the case of vanishing mean. Electron. Comm. Probab. 12, 2007, 248-258.

[38] O'Rourke, S.; Soshnikov, A. Product of independent non-Hermitian random matrices. Preprint, arXiv:1012.4497.

[39] Saff E. B.; Totik V. Logarithmic potentials with external fields. Appendix B by Thomas Bloom. Grundlehren der Mathematischen Wissenschaften [Fundamental Principles of Mathematical Sciences], 316. Springer-Verlag, Berlin, 1997.

[40] Speicher, R. Free probability theory. Preprint, arXiv:0911.0087.

[41] Tao, T.; Vu, V. Random matrices: universality of ESDs and the circular law. With an appendix by Manjunath Krishnapur. Ann. Probab. 38, no. 5, 2010, 2023-2065.

[42] Timushev D., Tikhomirov A. On the asymptotic distribution of singular values of powers products of sparse random matrices. Izvestia Komi Science Center of Ural Division of RAS, vol.13, 2013, 10-17.

[43] Tikhomirov, A. N. On the asymptotics of the spectrum of the product of two rectangular random matrices. (Russian) Sibirsk. Mat. Zh. 52, no. 4, 2011, 936-954; translation in Sib. Math. J. 52, no. 4, 2011, 747-762.

[44] Tikhomirov, A. N. Asymptotic distribution of the singular numbers for spherical ensemble matrices. (Russian) Mat. Tr. 16, no. 2, 2013, 169-200.

[45] Voiculescu D. Multiplication of certain noncommuting random variables. J. Operator Theory 18, 1987, 223-235.

[46] Voiculescu, D. Lectures on free probability theory. In book: "Lectures on Probability Theory and Statistics (Saint-Flour, 1998)", vol. 1738 of Lecture Notes in Math., 279-349.

[47] Voiculescu, D. Limit laws for Random matrices and free products. Invent. math., vol. 104, 1991, 201-220.

[48] Wigner, E. P. Characteristic vectors of bordered matrices with infinite dimensions. Ann. of Math. (2) 62, 1955, 548-564. 